NBER WORKING PAPER SERIES

\title{
CROSS-SECTIONAL DISPERSION OF RISK IN TRADING TIME
}

\author{
Torben G. Andersen \\ Martin Thyrsgaard \\ Viktor Todorov \\ Working Paper 26329 \\ http://www.nber.org/papers/w26329
NATIONAL BUREAU OF ECONOMIC RESEARCH
1050 Massachusetts Avenue
Cambridge, MA 02138
September 2019

Research partially supported by NSF grant SES-1530748. We would like to thank participants at various conferences and seminars for helpful comments and suggestions. The views expressed herein are those of the authors and do not necessarily reflect the views of the National Bureau of Economic Research.

At least one co-author has disclosed a financial relationship of potential relevance for this research. Further information is available online at http://www.nber.org/papers/w26329.ack

NBER working papers are circulated for discussion and comment purposes. They have not been peer-reviewed or been subject to the review by the NBER Board of Directors that accompanies official NBER publications.

(C) 2019 by Torben G. Andersen, Martin Thyrsgaard, and Viktor Todorov. All rights reserved. Short sections of text, not to exceed two paragraphs, may be quoted without explicit permission provided that full credit, including $(\odot$ notice, is given to the source. 
Cross-Sectional Dispersion of Risk in Trading Time

Torben G. Andersen, Martin Thyrsgaard, and Viktor Todorov

NBER Working Paper No. 26329

September 2019

JEL No. C51,C52,G12

\begin{abstract}
$\underline{\text { ABSTRACT }}$
We study the temporal behavior of the cross-sectional distribution of assets' market exposure, or betas, using a large panel of high-frequency returns. The asymptotic setup has the sampling frequency of the returns increasing to infinity, while the time span of the data remains fixed, and the cross-sectional dimension is fixed or increasing. We derive a Central Limit Theorem (CLT) for the cross-sectional beta dispersion at a point in time, enabling us to test whether this quantity varies across the trading day. We further derive a functional CLT for the dispersion statistics, allowing us to test if the beta dispersion, as a function of time-of-day, changes across days. We extend this further by developing inference techniques for the entire cross-sectional beta distribution at fixed points in time. We demonstrate, for constituents of the S\&P 500 index, that the beta dispersion is elevated at the market open, gradually declines over the trading day, and is less than half the original value by the market close. The intraday beta dispersion pattern also changes over time and evolves differently on macroeconomic announcement days. Importantly, we find that the intraday variation in market betas is a source of priced risk.
\end{abstract}

Torben G. Andersen

Kellogg School of Management

Northwestern University

2001 Sheridan Road

Evanston, IL 60208

and NBER

t-andersen@kellogg.northwestern.edu

Martin Thyrsgaard

Kellogg School of Management

Northwestern University

2211 Campus dr

Evanston, IL 60208

martin.thyrsgaard@kellogg.northwestern.edu
Viktor Todorov

Department of Finance

Kellogg School of Management

Northwestern University

2001 Sheridan Road

Evanston, IL

v-todorov@kellogg.northwestern.edu 


\section{Introduction}

Measuring assets' exposure to systematic risk, including the sensitivity to the overall market, plays a central role in the implementation and testing of asset pricing models. Indeed, measurement error in the estimated betas, i.e., covariances of asset returns with the systematic risk factors, can have nontrivial consequences for assessing the ability of asset pricing models to explain the cross-section of asset prices, see, e.g., Shanken (1992), Jaganathan and Wang (1998), Kan and Zhang (1999), Gospodinov, Kan, and Robotti (2009) and Kleibergen (2009). The standard approach of measuring betas is based on running linear regressions using daily or lower frequency data. This requires the use of periods spanning multiple years to control the size of measurement errors in the estimated betas. However, asset betas change over time, and this can be crucial for applications, i.e., an asset pricing model may hold only conditionally, see, e.g., Hansen and Richard (1987).

One way to accommodate time variation in betas is to model them as functions of observables such as firm characteristics, macro variables and systematic risk factors, see, e.g., Shanken (1990), Jagannathan and Wang (1996), Ferson and Harvey (1999), Connor, Hagmann, and Linton (2012), Gagliardini, Ossola, and Scaillet (2016), among others. An alternative (nonparametric) approach, following Barndorff-Nielsen and Shephard (2004a), is to make use of high-frequency returns. Intraday data hold the promise of attaining the identical precision in beta estimation as from low frequency data, but over significantly shorter time windows. The increased liquidity in financial markets renders such an approach practically feasible; indeed, Bollerslev, Li, and Todorov (2016) demonstrate the advantage of using high-frequency betas for cross-sectional asset pricing.

Prior studies using intraday data to estimate beta typically ignore any potential intraday variation in betas by averaging across the trading day. They either compute beta as a ratio of the daily integrated covariance of the asset with the systematic factor divided by the daily integrated variance of the systematic factor (as in Barndorff-Nielsen and Shephard (2004a)), or they aggregate betas estimated over local blocks (as in Mykland and Zhang (2009) and Li, Todorov, and Tauchen (2017)) across the day. In either case, the objective is to enhance the precision of the estimates. The premise is that intraday variation in beta is nonexistent or that it is immaterial for asset pricing. Given the extensive evidence of pronounced intraday variation in second-order return moments, it is natural to ask if this assumption is empirically justified. As an illustration, in Figure 1, we plot estimates of the market beta for two large stocks (Caterpillar and Johnson \& Johnson) using high-frequency returns from distinct parts of the trading day. The figure suggests that market betas

vary in a highly systematic manner. The betas of both stocks converge strongly towards unity-the cross-sectional population average of market betas-over the course of the trading day. 

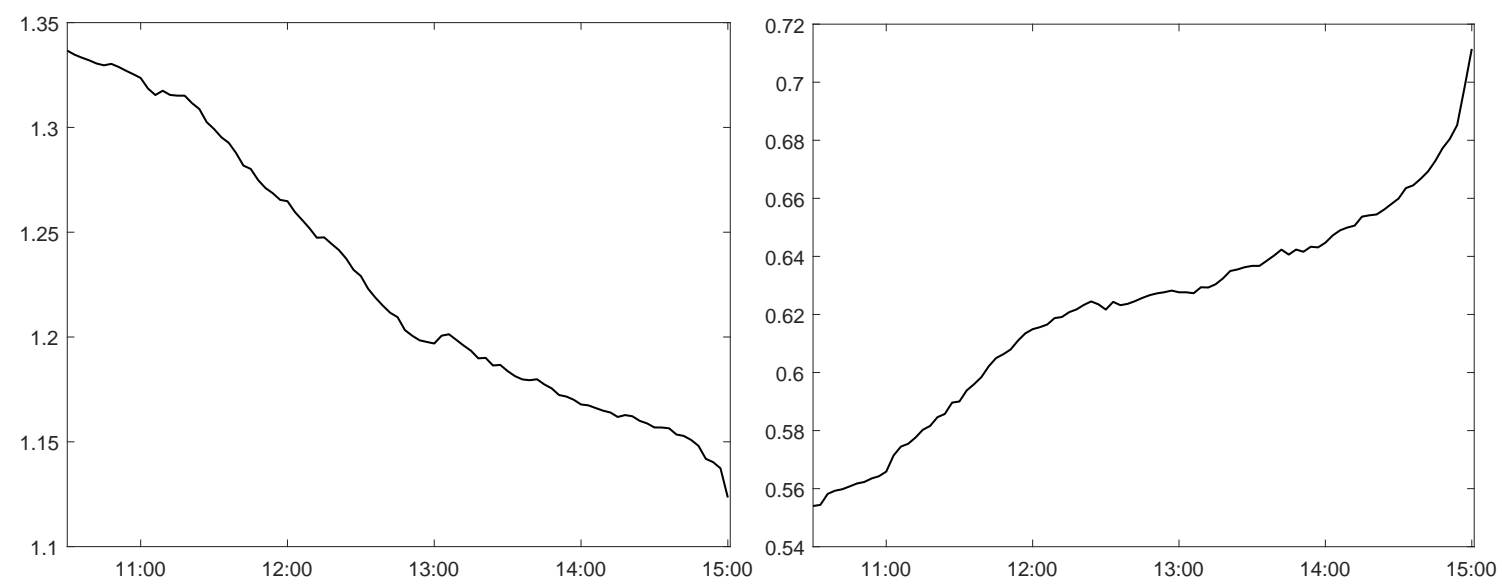

Figure 1: Intraday Variation in Market Betas. The betas are computed over local windows of two hours using 3-minute return data, according to equation (18), and averaged over the full sample of 2010-2018. The left panel represents Caterpillar and the right is for Johnson \& Johnson.

Is the above evidence of intraday variation in market betas statistically significant? Does it hold more generally in the cross-section of stocks? The goal of the current paper is to develop econometric tools for answering such questions, characterizing the pattern of intraday variation in betas, and studying its role in explaining the cross-sectional variation in asset prices.

Figure 1 suggests that, if present, the time variation in market beta takes on a particular form. Specifically, the cross-sectional dispersion of betas may decline monotonically over the trading day. Formally, we define this quantity (the cross-sectional dispersion of betas) at each point in time as,

$$
D_{t, \kappa}^{N}=\frac{1}{N} \sum_{j=1}^{N}\left(\beta_{t-1+\kappa}^{(j)}-1\right)^{2}, \quad t \in \mathbb{N}_{+}, \quad \kappa \in[0,1] .
$$

Here, $t$ indicates the trading day, $\kappa$ denotes the timing within the trading day (our time unit is one day), and $N$ is the number of assets in the cross-section. We construct estimates of $D_{t, \kappa}^{N}$ and develop the feasible limit theory needed for formal econometric inference of such objects. Our measures are constructed from a panel of high-frequency returns on a large cross-section of assets. The asymptotic setting is one in which the mesh of the observation grid shrinks to zero while the time span of the data remains fixed. The size of the cross-section may remain fixed or increase along with the sampling frequency. In the latter case, our inference is for the cross-sectional limit of $D_{t, \kappa}^{N}$ which, in general, is a random quantity.

We form our measure of the cross-sectional dispersion in market beta from the ratio of a local quadratic covariation estimate for the asset and market return divided by a local estimate for the quadratic return variation of the market. If the size of the cross-section grows asymptotically, then the associated limit distribution, evaluated at a fixed and finite set of points during the trading 
day (as indicated by the values of $\kappa$ ) is determined solely by the systematic risk factors in the asset returns, where we allow for an arbitrary fixed number (unknown to the econometrician) of latent systematic risk factors beyond the market. In contrast, if the size of the cross-section remains fixed, then the idiosyncratic risks will also impact the limit distribution of our statistic. Importantly, however, our feasible inference procedures are valid both for a fixed or increasing $N$. Moreover, the inference is conducted in a way that does not require knowledge of the number of systematic factors in the returns or the corresponding factor loadings.

This limit result enables one to compare $D_{t, \kappa}^{N}$ across a fixed set of distinct values for $\kappa$. If the dispersion changes during the day, however, it is natural to view $D_{t, \kappa}^{N}$ as a function of $\kappa$, and then ask whether this function varies over time or for days that include prescheduled macroeconomic announcements, when the information flow may differ from that on regular trading days. To address such questions, we go on to develop functional limit results for $D_{t, \kappa}^{N}$, with $D_{t, \kappa}^{N}$ viewed as a function of $\kappa$. Directly deriving the limiting behavior for estimates of $D_{t, \kappa}^{N}$ as functions of $\kappa$ is, however, difficult because this convergence does not hold uniformly in $\kappa$. Instead, we adopt an alternative approach, taking advantage of the one-to-one mapping between a function and its Fourier transform. This is convenient, as the Fourier transform of estimates for $D_{t, \kappa}^{N}$ consists of weighted sums of block estimates for the dispersion over the day, and their limit distribution is mixed Gaussian. Furthermore, we show that the convergence of these estimated Fourier transforms is uniform in their argument, thus delivering a functional convergence result enabling inference about $D_{t, \kappa}^{N}$ as a function of $\kappa$.

We extend the above analysis by developing inference for the full cross-sectional distribution of betas at a given point in time. Specifically, we derive a functional limit result for an estimate of the characteristic function of the cross-sectional market beta distribution at fixed points in time. This limit result can be used to test if there are other changes in the cross-sectional distribution of betas, not fully captured by the cross-sectional beta dispersion. In addition, through Fourier inversion, we may exploit this result to recover the density of the cross-sectional beta distribution at any given point in time nonparametrically.

We assess the finite sample performance of the new econometric tools through simulations from a model that mimics key features of the data used in our empirical study. The Monte Carlo analysis confirms that our limit theory provides a satisfactory basis for finite-sample inference. Our empirical application is based on the constituents of the S\&P 500 index over the sample period, 2010-2018. The estimated $D_{t, \kappa}^{N}$ for the full sample as well as select subsamples strongly reject the hypothesis of a constant cross-sectional dispersion of market betas across the trading day. Consistent with 
Figure 1, we find the highest beta dispersion at the market open, followed by a gradual decay during the trading day. That is, high/low beta stocks, with betas above/below unity, tend to have a downward/upward sloping market beta trajectories across the trading day. This intraday pattern holds throughout the sample, with the decline of the cross-sectional beta dispersion during the trading hours turning even more pronounced towards the latter part of the sample. Consistent with an information-based explanation for the observed pattern in betas, we also find that the behavior of $D_{t, \kappa}^{N}$, as a function of $\kappa$, changes around prescheduled macroeconomic announcements, exemplified by the closing day of regular Federal Open Market Committee (FOMC) meetings. During those days, the drop in $D_{t, \kappa}^{N}$ occurs in the immediate aftermath of the announcement, and $D_{t, \kappa}^{N}$ then remains flat for the remainder of the trading day.

Given the stability and strong statistical significance of the intraday variation in market betas, one may wonder whether it matters for asset pricing. To address this question, we construct a number of tests based on a simple sorting scheme. At the beginning of each trading day, we sort the stocks in the cross-section according to their beta at the market open. We then form a portfolio long the stocks in the bottom one-third and short the stocks in the top one-third of this beta distribution. We scale the long positions so that the overall beta of the portfolio is zero at the market open. We hold the portfolio over the full trading day and close the position at the market close. Because of the systematic intraday variation in the market betas, this portfolio does not remain market-neutral, but gradually becomes more positively exposed to market risk as the trading day unfolds. If this intraday beta variation is unpriced, however, this portfolio should not earn a risk premium. On the contrary, we find strong evidence against this hypothesis, suggesting that this intraday beta variation is a source of priced risk. We conduct various robustness checks that further corroborate this conclusion.

The rest of the paper is organized as follows. We first introduce setup and notation in Section 2. We then construct our measures of cross-sectional dispersion in market betas in Section 3 . This section also develops the feasible limit theory for our dispersion statistics. We apply these asymptotic results in Section 4 to construct tests for changes in the beta dispersion both within the trading day and across trading days. Section 5 extends the theory by developing inference for the entire cross-sectional beta distribution. We assess the finite-sample properties of the econometric tools through simulations in Section 6. Section 7 contains our empirical analysis, and Section 8 concludes. Finally, the proofs are provided in Section 9. 


\section{Setup and Notation}

We first introduce the basic setup. We consider a set of stocks, indexed by $j=1, \ldots, N$, whose prices are defined on some filtered probability space $\left(\Omega, \mathcal{F},\left(\mathcal{F}_{t}\right)_{t \geq 0}, \mathbb{P}\right)$. The market portfolio is assigned the index $j=0$. The evolution of these processes is given by,

$$
\begin{aligned}
X_{t}^{(0)} & =X_{0}^{(0)}+\int_{0}^{t} \alpha_{s}^{(0)} d s+\int_{0}^{t} \sigma_{s}^{(0)} d W_{s}^{(0)}+\sum_{s \leq t} \Delta X_{s}^{(0)}, \\
X_{t}^{(j)}= & X_{0}^{(j)}+\int_{0}^{t} \alpha_{s}^{(j)} d s+\int_{0}^{t} \beta_{s}^{(j)} \sigma_{s}^{(0)} d W_{s}^{(0)}+\int_{0}^{t} \gamma_{s}^{(j)} d B_{s} \\
& +\int_{0}^{t} \widetilde{\sigma}_{s}^{(j)} d \widetilde{W}_{s}^{(j)}+\sum_{s \leq t} \Delta X_{s}^{(j)}, \quad j=1, . ., N,
\end{aligned}
$$

where $W_{t}^{(0)},\left(\widetilde{W}_{t}^{(j)}\right)_{j=1, \ldots, N}$ and $B_{t}$ are independent Brownian motions, the dimension of $B_{t}$ is $r \times 1$ and the rest of the Brownian motions are univariate; $\left\{\alpha_{t}^{(j)}\right\}_{j=0,1, \ldots, N}, \sigma_{t}^{(0)},\left\{\beta_{t}^{(j)}\right\}_{j=1, \ldots, N}$, $\left\{\widetilde{\sigma}_{t}^{(j)}\right\}_{j=1, \ldots, N}$ and $\left\{\gamma_{t}^{(j)}\right\}_{j=1, \ldots, N}$ are processes with càdlàg paths, with $\gamma_{t}^{(j)}$ being $1 \times r$ dimensional, and the remainder are scalar-valued functions of time. Finally, for any process $Y, \Delta Y_{t}=Y_{t}-Y_{t-}$ denotes the size of a jump at time $t$. The technical assumptions concerning the processes appearing in equations (2)-(3) are provided in the appendix.

The return dynamics implied by model (2)-(3) nests most existing models used in cross-sectional asset pricing. In particular, we allow for an arbitrary number of systematic factors, and the factor loadings may be time-varying. Apart for the market index, the systematic factors are latent and their number $r$ unknown to the econometrician. Similarly, except for regularity type conditions, the time-varying factor loadings and the time-varying stochastic volatilities are left unrestricted. Idiosyncratic (diffusive) risk in asset prices is captured by the independent Brownian motions $\left(\widetilde{W}_{t}^{(j)}\right)_{j=1, \ldots, N}$. Likewise, the dependence structure of the jumps in the cross-section is also unconstrained. If asset price jumps have the identical beta with respect to market jumps as the diffusive beta, $\beta_{t}^{(j)}$, they may be included in the inference. We do not impose this restriction, so we eliminate returns containing (identified) jumps when constructing our statistics.

We focus on the evolution of the cross-sectional distribution of market betas over time. Denoting the continuous part of the (predictable) quadratic variation for two semimartingales $X$ and $Y$ by $\langle X, Y\rangle^{c}$ (see, e.g., Section I.4 in Jacod and Shiryaev (2003)), we may define the market betas as,

$$
\beta_{t}^{(j)}=\frac{d\left\langle X^{(j)}, X^{(0)}\right\rangle_{t}^{c}}{d\left\langle X^{(0)}, X^{(0)}\right\rangle_{t}^{c}}, \quad t \in \mathbb{R}_{+}, \quad j=1, \ldots, N
$$

Of course, the analysis that follows can be trivially extended to study the cross-sectional distribution of factor loadings with respect to other (observable) systematic risk factors. 


\section{Inference for the Cross-Sectional Dispersion of Market Betas}

This section introduces our cross-sectional dispersion measures for market betas and derives an associated feasible limit theory. The inference is based on discrete observations of $\left\{X^{(j)}\right\}_{j=0,1, \ldots, N}$ at equidistant times $0, \frac{1}{n}, \frac{2}{n}, \ldots, T$, where $T$ refers to the time span of our data, which is fixed throughout, the integer $n$ denotes the number of times we sample within a unit interval, $\Delta_{n}=1 / n$ signifies the length of the sampling interval, and the high-frequency increment of $X^{(j)}$ is given by,

$$
\Delta_{t, i}^{n} X^{(j)}=X_{(t-1)+i / n}^{(j)}-X_{(t-1)+(i-1) / n}^{(j)}, \quad t \in \mathbb{N}_{+}, \quad i=1, \ldots, n, j=0,1, \ldots, N
$$

As noted in the introduction, we measure time in units of one day. The notation above, therefore, implicitly assumes that we sample at high frequency throughout the full day. In practice, of course, the day consists of an active trading session and an overnight period with no, or only limited, trading. We may accommodate this feature by splitting the unit interval $[t-1, t]$ into a trading part $[t-1, t-1+\bar{\kappa}]$ and an overnight period $[t-1+\bar{\kappa}, t]$, for some $\bar{\kappa} \in(0,1]$. We may then assume we utilize high-frequency observations only during the interval $[t-1, t-1+\bar{\kappa}]$, i.e., that we sample at $t-1, t-1+\frac{1}{n}, \ldots, t-1+\frac{\lfloor\bar{\kappa} n\rfloor}{n}$, for $t=1, \ldots,\lfloor T\rfloor$. However, to simplify notation, henceforth, we set $\bar{\kappa}=1$, so we exclude the overnight periods. It is evident that our results, trivially, may be extended to allow for $\bar{\kappa}<1$.

\subsection{Estimates of the Market Beta Dispersion}

We start by forming estimates for the market beta dispersion at any given point in time. In constructing our statistics, we rely on a standard truncation approach (see, e.g., Mancini (2001, 2009), Jacod and Protter (2012)) to eliminate the jumps in the (partially) observed sample path of the assets. More specifically, we truncate the increments of $X^{(k)}$ via,

$$
\nu_{t, n}^{(j)} \asymp \Delta_{n}^{\varpi}, \quad \varpi \in(0,1 / 2), \quad t \in \mathbb{N}_{+}, \quad j=0,1, \ldots, N
$$

In the Monte Carlo study and the empirical analysis we provide additional detail on the particular choice of $\nu_{t, n}^{(j)}$. Exploiting the above notation, we define the sets,

$$
\mathcal{A}_{t, i}^{(j)}=\left\{\left|\Delta_{t, i}^{n} X^{(j)}\right| \leq \nu_{t, n}^{(j)}\right\}, \quad \mathcal{A}_{t, i}^{(j, l)}=\left\{\left|\Delta_{t, i}^{n} X^{(j)}\right| \leq \nu_{t, n}^{(j)} \cap\left|\Delta_{t, i}^{n} X^{(l)}\right| \leq \nu_{t, n}^{(l)}\right\},
$$

for $j, l=0,1, \ldots, N$. Our statistics will be computed on these sets.

To construct an estimate for the cross-sectional dispersion of betas at a given point in time, $D_{t, \kappa}^{N}$, we must account for the fact that both the market beta and the asset volatilities are stochastic and change over time. As a result, we construct our measures on an interval whose length shrinks 
as we sample more frequently. Specifically, we use a local window of $k_{n}$ high-frequency increments, for a sequence $k_{n}$ satisfying,

$$
k_{n} \asymp n^{\varrho}, \quad \varrho \in(0,1) .
$$

The sets of indices for the price increments over which we compute our measures on a given trading day is then denoted,

$$
\mathcal{I}_{\kappa}^{n}=\left\{\lfloor\kappa n\rfloor-k_{n}+1, \ldots,\lfloor\kappa n\rfloor\right\}, \quad \widetilde{\mathcal{I}}_{\kappa}^{n}=\left\{\lfloor\kappa n\rfloor-k_{n}+2, \ldots,\lfloor\kappa n\rfloor\right\}, \quad \kappa \in[0,1] .
$$

To compute market betas and their cross-sectional dispersion, we require estimates for the quadratic (co-)variation. The continuous part of the quadratic variation for an asset is estimated by,

$$
\widehat{V}_{t, \kappa}^{(j, l)}=\frac{n}{\left|\mathcal{I}_{\kappa}^{n}\right|} \sum_{i \in \mathcal{I}_{\kappa}^{n}}\left(\Delta_{t, i}^{n} X^{(j)}\right)^{2} 1_{\left\{\mathcal{A}_{t, i}^{(j, l)}\right\}}, \quad j, l=0,1, \ldots, N, \quad t \in \mathbb{N}_{+}, \kappa \in[0,1] .
$$

If the objective is to measure the continuous quadratic variation of asset $j$, it is natural to set $l=j$ in $\widehat{V}_{t, \kappa}^{(j, l)}$. However, for measuring beta, it makes more sense to compute the quadratic variation and covariation used in its construction on the identical set of increments. Of course, the jump times of a semimartingale on a finite interval are of Lebesgue measure zero, and hence whether we set $l=j$ or $l \neq j$ makes no difference for the asymptotic analysis that follows.

We next define a measure for the difference between the asset covariation with the market and the market variation as follows,

$$
\begin{aligned}
\widehat{C}_{t, \kappa}^{(j)}(2)=\frac{1}{2} \frac{n}{\left|\widetilde{\mathcal{I}}_{\kappa}^{n}\right|} \sum_{i \in \widetilde{\mathcal{I}}_{\kappa}^{n}} & {\left[\left(\Delta_{t, i-1}^{n} X^{(j)}+\Delta_{t, i}^{n} X^{(j)}-\Delta_{t, i-1}^{n} X^{(0)}-\Delta_{t, i}^{n} X^{(0)}\right)\right.} \\
& \left.\times\left(\Delta_{t, i-1}^{n} X^{(0)}+\Delta_{t, i}^{n} X^{(0)}\right) 1_{\left\{\mathcal{A}_{t, i-1}^{(j, 0)} \cap \mathcal{A}_{t, i}^{(j, 0)}\right\}}\right], \quad t \in \mathbb{N}_{+}, \quad \kappa \in[0,1],
\end{aligned}
$$

where $j=1, \ldots, N$. Note that we use a coarser frequency in the construction of $\widehat{C}_{t, \kappa}^{(j)}(2)$, as we double the length of the high-frequency increments used in $\widehat{C}_{t, \kappa}^{(j)}(2)$ compared to those for $\widehat{V}_{t, \kappa}^{(j, l)}$ (the use of an even coarser grid for the covariance measure is readily accommodated). The reason is twofold. First, from a theoretical perspective, the coarser frequency avoids a potential "degeneracy." If we were to use the identical frequency for $\widehat{C}_{t, \kappa}^{(j)}(2)$ and $\widehat{V}_{t, \kappa}^{(j, l)}$ then, in the absence of other systematic risk factors beyond the market return, the leading term for the error of our estimate of the dispersion measure will stem from the time variation in betas and stochastic volatilities over the local window. The behavior of this error is difficult to characterize in our general nonparametric setup. Given existing empirical evidence for multiple systematic risk factors in asset returns, the degenerate scenario is not likely to be of practical relevance. Nonetheless, we define our statistics such that this issue is avoided, with the cost being a slight loss of efficiency in our estimator if the market is 
the sole systematic risk factor. Second, from a practical perspective, the use of a coarser frequency in $\widehat{C}_{t, \kappa}^{(j)}(2)$ helps minimize the impact of potential asynchroneity effects on the statistics due to the lower liquidity of individual assets relative to the market index.

Our local measure of cross-sectional market beta dispersion, $\widehat{D}_{t, \kappa}^{N}$, is now simply given by,

$$
\widehat{D}_{t, \kappa}^{N}=\frac{1}{N} \sum_{j=1}^{N}\left(\left(\frac{\widehat{C}_{t, \kappa}^{(j)}(2)}{\widehat{V}_{t, \kappa}^{(0, j)}}\right)^{2} 1_{\left\{\widehat{V}_{t, \kappa}^{(0, j)}>\alpha_{n}\right\}}\right), \quad \kappa \in[0,1],
$$

where

$$
\alpha_{n} \asymp 1 / \log (n) .
$$

Because the estimate of the beta dispersion involves division by $\widehat{V}_{t, \kappa}^{(0, j)}$, which is not bounded away from zero in finite samples, we impose a lower bound for the local estimate of market variance. This bound does not have an asymptotic effect on the statistic or its limit distribution.

It is easy to show (and it also follows from our convergence results in the next section) that as $n \rightarrow \infty$ and $N \rightarrow \bar{N}$, for some $\bar{N} \in(0, \infty]$, we have the following convergence in probability under Assumption A, given in the appendix,

$$
\widehat{D}_{t, \kappa}^{N}-D_{t, \kappa}^{N} \stackrel{\mathbb{P}}{\longrightarrow} 0
$$

We note that we allow for the size of the cross-section either to remain fixed or to increase as we sample more frequently. In the latter case, $D_{t, \kappa}^{N}$ typically converges, under suitable assumptions, to a cross-sectional limit, which may still be a random number. Allowing for $\bar{N}$ to be finite or infinite is convenient from an empirical point of view, as one need not take a stand on whether the cross-sectional dimension of the return panel is sufficiently large relative to the sampling frequency to justify one finite-sample approximation versus another.

We next introduce a few measures that are used in the construction of a bias-correction term for $\widehat{D}_{t, \kappa}^{N}$ as well as an estimate for its asymptotic variance. Towards this end, we split the set $\mathcal{I}_{\kappa}^{n}$ into the following two "even" and "odd" subsets,

$$
\mathcal{E}_{\kappa}^{n}=\left\{\lfloor\kappa n\rfloor-2\left\lfloor\frac{k_{n}-1}{2}\right\rfloor,\lfloor\kappa n\rfloor-2\left\lfloor\frac{k_{n}-3}{2}\right\rfloor, \ldots,\lfloor\kappa n\rfloor-2,\lfloor\kappa n\rfloor\right\}, \quad \mathcal{O}_{\kappa}^{n}=\mathcal{I}_{\kappa}^{n} \backslash \mathcal{E}_{\kappa}^{n},
$$

and define the following two measures for $j=1, \ldots, N$,

$$
\begin{gathered}
\breve{C}_{t, \kappa}^{(j)}=\frac{n}{\left|\mathcal{I}_{\kappa}^{n}\right|} \sum_{i \in \mathcal{I}_{\kappa}^{n}}\left[\left(\Delta_{t, i}^{n} X^{(j)}-\Delta_{t, i}^{n} X^{(0)}\right) \Delta_{t, i}^{n} X^{(0)} 1_{\left\{\mathcal{A}_{t, i}^{(j, 0)}\right\}}\left(1_{\left\{i \in \mathcal{O}_{\kappa}^{n}\right\}}-1_{\left\{i \in \mathcal{E}_{\kappa}^{n}\right\}}\right)\right], \\
\breve{V}_{t, \kappa}^{(0, j)}=\frac{n}{\left|\mathcal{I}_{\kappa}^{n}\right|} \sum_{i \in \mathcal{I}_{\kappa}^{n}}\left[\left(\Delta_{t, i}^{n} X^{(0)}\right)^{2} 1_{\left\{\mathcal{A}_{t, i}^{(j, 0)}\right\}}\left(1_{\left\{i \in \mathcal{O}_{\kappa}^{n}\right\}}-1_{\left\{i \in \mathcal{E}_{\kappa}^{n}\right\}}\right)\right] .
\end{gathered}
$$


The motivation behind these definitions of the two statistics is the following. By subtracting consecutive summands in $\breve{C}_{t, \kappa}^{(j)}$ and $\breve{V}_{t, \kappa}^{(0, j)}$, we cancel their (conditional) mean which, although random, is locally constant (i.e., approximately constant over the short time window over which $\widehat{C}_{t, \kappa}^{(j)}(2)$ and $\widehat{V}_{t, \kappa}^{(0, j)}$ are computed). After this centering, the consecutive summands are approximately independent and possess a symmetric distribution. Therefore, $\breve{C}_{t, \kappa}^{(j)}$ and $\breve{V}_{t, \kappa}^{(0, j)}$ can be used to measure the variability in $\widehat{C}_{t, \kappa}^{(j)}(2)$ and $\widehat{V}_{t, \kappa}^{(0, j)}$. This is very convenient for our setup, in which the size of the cross-section $N$ can increase to infinity asymptotically, as we avoid estimation of the additional systematic factors driving the asset returns as well as the loadings on them. Similarly, we do not need to make an assumption or impose a bound on the number of systematic factors driving the returns, which is typically the case when making inference for factor models.

This approach of computing the asymptotic variance is reminiscent of the so-called observed asymptotic variance proposed recently by Mykland and Zhang (2017), although there are a couple of nontrivial differences. First, in our setting $N$ may be asymptotically increasing, which complicates matters, because of the (unknown) factor structure in the returns. Second, we use statistics from "even" and "odd" increments in generating the "observed asymptotic variance," unlike Mykland and Zhang (2017), who use successive blocks. Our procedure, therefore, generates less of a bias in situations where the estimand may vary across the local block (which will be the case for our dispersion measures).

Finally, in the construction of the bias-correction term as well as the asymptotic variance, we need a local estimate of the market betas. We use the following,

$$
\widehat{\beta}_{t, \kappa}^{(j)}=\frac{\sum_{i \in \mathcal{I}_{\kappa}^{n}} \Delta_{t, i}^{n} X^{(j)} \Delta_{t, i}^{n} X^{(0)} 1_{\left\{\mathcal{A}_{t, i}^{(j, 0)}\right\}}}{\sum_{i \in \mathcal{I}_{\kappa}^{n}}\left(\Delta_{t, i}^{n} X^{(0)}\right)^{2} 1_{\left\{\mathcal{A}_{t, i}^{(j, 0)}\right\}}}, j=1, \ldots, N
$$

and note that, as in the construction of $\widehat{D}_{t, \kappa}^{N}$, we could use a coarser frequency for computing the covariation in the numerator. An estimate for the sample variance of $\widehat{\beta}_{t, \kappa}^{(j)}$ is given by,

$$
\widehat{V}_{\beta, t, \kappa}^{(j)}=\frac{\sum_{i \in \mathcal{I}_{\kappa}^{n}}\left[\left(\Delta_{t, i}^{n} X^{(0)}\right)^{2}\left(\Delta_{t, i}^{n} X^{(j)}-\widehat{\beta}_{t, \kappa}^{(j)} \Delta_{t, i}^{n} X^{(0)}\right)^{2} 1_{\left\{\mathcal{A}_{t, i}^{(j, 0)}\right\}}\right]}{\left(\sum_{i \in \mathcal{I}_{\kappa}^{n}}\left(\Delta_{t, i}^{n} X^{(0)}\right)^{2} 1_{\left\{\mathcal{A}_{t, i}^{(j, 0)}\right\}}\right)^{2}}, j=1, \ldots, N
$$

The nonlinear transformation of $\widehat{C}_{t, \kappa}^{(j)}(2)$ and $\widehat{V}_{t, \kappa}^{(0, j)}$ in the construction of the dispersion measure $\widehat{D}_{t, \kappa}^{N}$ introduces an (upward) bias of asymptotic order $O_{p}\left(1 / k_{n}\right)$. It may be estimated via,

$$
\left.\widehat{B}_{t, \kappa}^{N}=\frac{1}{N} \sum_{j=1}^{N}\left(\left[-\frac{\left(\widehat{\beta}_{t, \kappa}^{(j)}-1\right)^{2}-\widehat{V}_{\beta, t, \kappa}^{(j)}\left(\breve{V}_{t, \kappa}^{(0, j)}\right)^{2}+\frac{3}{2\left(\widehat{V}_{t, \kappa}^{(0, j)}\right)^{2}}\left(\breve{C}_{t, \kappa}^{(j)}\right)^{2}}{(j)}\right)^{2}\right] 1_{\left\{\widehat{V}_{t, \kappa}^{(0, j)}>\alpha_{n}\right\}}\right) .
$$


$\widehat{B}_{t, \kappa}^{N}$ provides an estimate of the second-order terms in the Taylor expansion of $\widehat{D}_{t, \kappa}^{N}$, viewed as a function of $\widehat{C}_{t, \kappa}^{(j)}(2)$ and $\widehat{V}_{t, \kappa}^{(0, j)}$. These terms, in turn, depend on the variance of $\widehat{C}_{t, \kappa}^{(j)}(2)$ and $\widehat{V}_{t, \kappa}^{(0, j)}$, which we estimate using $\breve{V}_{t, \kappa}^{(0, j)}$ and $\breve{C}_{t, \kappa}^{(j)}$. Because of the different frequency used in computing $\widehat{C}_{t, \kappa}^{(j)}(2)$ and $\widehat{V}_{t, \kappa}^{(0, j)}$, we need to weight $\breve{V}_{t, \kappa}^{(0, j)}$ and $\breve{C}_{t, \kappa}^{(j)}$ appropriately to account for the different contribution to the bias term of, on the other hand, $W^{(0)}$, and, on the one hand, $B$ and $\left\{\widetilde{W}^{(j)}\right\}_{j=1, \ldots, N}$, relative to the case where we rely on the identical frequency in constructing the measures.

Finally, our estimator of the asymptotic variance of $\widehat{D}_{t, \kappa}^{N}$ is given by

$$
\begin{aligned}
\widehat{\operatorname{Avar}}\left(\widehat{D}_{t, \kappa}^{N}\right) & =\frac{3}{2}\left(\frac{1}{N} \sum_{j=1}^{N}\left[\frac{2\left(\widehat{\beta}_{t, \kappa}^{(j)}-1\right)}{\widehat{V}_{t, \kappa}^{(0, j)}}\left(\breve{C}_{t, \kappa}^{(j)}-\left(\widehat{\beta}_{t, \kappa}^{(j)}-1\right) \breve{V}_{t, \kappa}^{(0, j)}\right) 1_{\left\{\widehat{V}_{t, \kappa}^{(0, j)}>\alpha_{n}\right\}}\right]\right)^{2} \\
& +\frac{1}{2}\left(\frac { 1 } { N } \sum _ { j = 1 } ^ { N } \left[\frac{\left.\left.2\left(\widehat{\beta}_{t, \kappa}^{(j)}-1\right)^{2}-2 \widehat{V}_{\beta, t, \kappa}^{(j)} \breve{V}_{t, \kappa}^{(0, j)} 1_{\left\{\widehat{V}_{t, \kappa}^{(0, j)}>\alpha_{n}\right\}}\right]\right)^{2}}{\widehat{V}_{t, \kappa}^{(0, j)}} .\right.\right.
\end{aligned}
$$

The discussion regarding the weights assigned to $\breve{V}_{t, \kappa}^{(0, j)}$ and $\breve{C}_{t, \kappa}^{(j)}$ in the construction of the bias terms above apply here for the construction of $\widehat{\operatorname{Avar}}\left(\widehat{D}_{t, \kappa}^{N}\right)$ as well.

\subsection{Feasible Limit Theory}

This section presents our limit results for the market beta dispersion statistic. We start with a feasible Central Limit Theorem (CLT) for $\widehat{D}_{t, \kappa}^{N}$ across different fixed points in time. In the theorems below, $\stackrel{\mathcal{L}-s}{\longrightarrow}$ denotes stable convergence in law, implying, importantly, that the convergence holds jointly with any $\mathcal{F}$-measurable random variable, see, e.g., Jacod and Shiryaev (2003).

Theorem 1. Suppose Assumptions $A$ and $B$ hold. Let $n \rightarrow \infty$ and $N \rightarrow \bar{N}$, for $\bar{N} \in(0, \infty]$, with $\varpi \in(1 / 4,1 / 2), \varrho \in(0,1 / 2)$ and $\varrho>2-4 \varpi$. For $\mathcal{K}$ an arbitrary finite set of points in $[0,1]$, and $\mathcal{T}$ a finite set of positive integers in $[0, T]$, we have,

$$
\left\{\frac{\widehat{D}_{t, \kappa}^{N}-\widehat{B}_{t, \kappa}^{N}-D_{t, \kappa}^{N}}{\sqrt{\widehat{\operatorname{Avar}}\left(\widehat{D}_{t, \kappa}^{N}\right)}}\right\}_{t \in \mathcal{T}, \kappa \in \mathcal{K}} \stackrel{\mathcal{L}-s}{\longrightarrow}\left\{Z_{t, \kappa}\right\}_{t \in \mathcal{T}, \kappa \in \mathcal{K}},
$$

where $\left\{Z_{t, \kappa}\right\}_{t \in \mathcal{T}, \kappa \in \mathcal{K}}$ is a sequence of standard normal random variables defined on an extension of the original probability space and independent of $\mathcal{F}$.

The limiting result above holds both when $N$ is fixed and when it increases to infinity asymptotically. There is no restriction on the relative growth of the sampling frequency and the size of the cross-section in Theorem 1. The asymptotic distribution of $\widehat{D}_{t, \kappa}^{N}$ is determined solely by the 
systematic factors in the asset returns when $N \rightarrow \infty$. This is because systematic risk, unlike idiosyncratic risk, is not averaged out in a cross-sectional aggregation. On the other hand, when $N$ is fixed, both systematic and idiosyncratic risks determine the limit distribution of $\widehat{D}_{t, \kappa}^{N}$. Importantly, the econometrician does not need to take a stand on which of the two asymptotic setups ( $N$ fixed or asymptotically increasing) is at work. Similarly, there is no need to estimate or impose a known upper bound on the number of systematic risk factors in the inference.

The asymptotic variance estimator, $\widehat{\operatorname{Avar}}\left(\widehat{D}_{t, \kappa}^{N}\right)$, is of order $O_{p}\left(1 / k_{n}\right)$, which implies that the rate of convergence of $\widehat{D}_{t, \kappa}^{N}$ is $\sqrt{k_{n}}$. In addition, the limit distribution of $\widehat{D}_{t, \kappa}^{N}$ is mixed Gaussian, as the probability limit of $k_{n} \widehat{\operatorname{Avar}}\left(\widehat{D}_{t, \kappa}^{N}\right)$, in general, will be a random variable. Finally, the dispersion measures computed at distinct points in time are asymptotically $\mathcal{F}$-conditionally independent.

As with other applications involving truncation-based estimates of volatility functionals, it is optimal to pick the truncation parameter $\varpi$ near $1 / 2$. When we do this, our restriction for the block size is very weak, suggesting a feasible CLT result will apply for a wide range of $k_{n}$. Of course, the bigger the (asymptotic) block size, the faster the rate of convergence for the dispersion measure $\widehat{D}_{t, \kappa}^{N}$.

Theorem 1 enables us to formally compare the cross-sectional market beta dispersion across different days as well as different times within the trading day. However, if the dispersion changes during the day, it is natural to study the behavior of $\widehat{D}_{t, \kappa}^{N}$ as a function of its time-of-day argument, $\kappa$. For this purpose, we now develop a functional convergence result for $\widehat{D}_{t, \kappa}^{N}$.

Using the one-to-one map between a function (in $L_{2}$ ) and its Fourier transform, we can characterize the behavior of the dispersion function during the trading day through its Fourier transform. Therefore, we introduce a family of functions to weight the dispersion measure across the different times of the trading day and analyze the associated functional convergence of the weighted dispersions within a family of weight functions. More specifically, suppose for every $u \in \mathcal{U}$, where $\mathcal{U}$ is a compact subset of $\mathbb{R}$, we have a weight function $\omega_{u}:[0,1] \rightarrow \mathbb{C}$. We then define our weighted (complex-valued) total dispersion measures via,

$$
\widehat{T D}_{t}^{N}(u)=\frac{1}{n-k_{n}+1} \sum_{i=k_{n}}^{n} \omega_{u}\left(i \Delta_{n}\right)\left(\widehat{D}_{t, i \Delta_{n}}^{N}-\widehat{B}_{t, i \Delta_{n}}^{N}\right), \quad t \in \mathbb{N}_{+}, \quad u \in \mathcal{U}
$$

The measure $\widehat{T D}_{t}^{N}(u)$ is a consistent estimator of,

$$
T D_{t}^{N}(u)=\int_{0}^{1} \omega_{u}(\kappa) D_{t, \kappa}^{N} d \kappa, \quad t \in \mathbb{N}_{+}, \quad u \in \mathcal{U},
$$

i.e., $\widehat{T D}_{t}^{N}(u)-T D_{t}^{N}(u)=o_{p}(1)$ uniformly in $u \in \mathcal{U}$. The following theorem states the CLT associated with this convergence in probability. 
Theorem 2. Suppose Assumptions $A$ and $B$ hold and, in addition, that the family of complexvalued functions $\left(\omega_{u}(z)\right)_{u \in \mathcal{U}}$ satisfies $\left|\omega_{u}(z)-\omega_{v}(z)\right| \leq K|u-v|$ and $\left|\omega_{u}(z)-\omega_{u}(w)\right| \leq K|z-w|$ for all $u, v \in \mathcal{U}$, where $\mathcal{U} \in \mathbb{R}$ is a compact set, $z, w \in[0,1]$, and $K>0$ is a constant. Let $n \rightarrow \infty$ and $N \rightarrow \bar{N}$ for $\bar{N} \in(0, \infty]$, with $\varpi \in(1 / 4,1 / 2), \varrho \in(1 / 3,1 / 2)$ and $\varrho>2-4 \varpi$. For $t \in \mathbb{N}_{+} \cap[0, T]$, we have,

$$
\sqrt{n-k_{n}+1}\left(\widehat{T D}_{t}^{N}(u)-T D_{t}^{N}(u)\right) \stackrel{\mathcal{L}-s}{\longrightarrow} Z_{t}(u)
$$

where the above convergence is for u-indexed processes under the uniform metric, and further $Z_{t}(u)$ is a complex-valued process, defined on an extension of the original probability space, which is $\mathcal{F}$-conditionally a centered Gaussian process on $\mathcal{U}$ with covariance and relation functions given by,

$$
\begin{aligned}
& \mathbb{E}\left(Z_{t}(u) Z_{t}(v)\right) \equiv \Sigma_{t}(u, v)=\operatorname{plim}_{N \rightarrow \bar{N}, n \rightarrow \infty} \widehat{\Sigma}_{t}(u, v), \quad \text { for } u, v \in \mathcal{U}, \\
& \mathbb{E}\left(Z_{t}(u) \overline{Z_{t}(v)}\right) \equiv \Xi_{t}(u, v)=\operatorname{plim}_{N \rightarrow \bar{N}, n \rightarrow \infty} \widehat{\Xi}_{t}(u, v), \quad \text { for } u, v \in \mathcal{U},
\end{aligned}
$$

where

$$
\begin{aligned}
& \widehat{\Sigma}_{t}(u, v)=\frac{k_{n}}{n-k_{n}+1} \sum_{i=1}^{n} \bar{\omega}_{u}^{n}(i) \bar{\omega}_{v}^{n}(i) \frac{\left(\widehat{\operatorname{Avar}}\left(\widehat{D}_{t,\left(i \vee k_{n}\right) \Delta_{n}}^{N}\right)+\widehat{\operatorname{Avar}}\left(\widehat{D}_{t,\left(\left(i+k_{n}\right) \wedge n\right) \Delta_{n}}^{N}\right)\right)}{2}, \\
& \widehat{\Xi}_{t}(u, v)=\frac{k_{n}}{n-k_{n}+1} \sum_{i=1}^{n} \bar{\omega}_{u}^{n}(i) \overline{\bar{\omega}_{v}^{n}(i)} \frac{\left(\widehat{\operatorname{Avar}}\left(\widehat{D}_{t,\left(i \vee k_{n}\right) \Delta_{n}}^{N}\right)+\widehat{\operatorname{Avar}}\left(\widehat{D}_{t,\left(\left(i+k_{n}\right) \wedge n\right) \Delta_{n}}^{N}\right)\right)}{2},
\end{aligned}
$$

with

$$
\bar{\omega}_{u}^{n}(i)= \begin{cases}\frac{1}{k_{n}} \sum_{j=0}^{i-1} \omega_{u}\left((i+j) \Delta_{n}\right), & i=1, \ldots, k_{n}, \\ \frac{1}{k_{n}} \sum_{j=0}^{k_{n}-1} \omega_{u}\left((i+j) \Delta_{n}\right), & i=k_{n}+1, \ldots, n,\end{cases}
$$

and, in the above, we set $\omega_{u}(s)=0$ for $s>1$. We further have,

$$
\sup _{u, v \in \mathcal{U}}\left|\widehat{\Sigma}_{t}(u, v)-\Sigma_{t}(u, v)\right| \stackrel{\mathbb{P}}{\longrightarrow} 0, \quad \sup _{u, v \in \mathcal{U}}\left|\widehat{\Xi}_{t}(u, v)-\Xi_{t}(u, v)\right| \stackrel{\mathbb{P}}{\longrightarrow} 0 .
$$

Moreover, for $\mathcal{T}$ a finite set of positive integers in $[0, T]$, the above convergence in law holds jointly for $t \in \mathcal{T}$, with $Z_{t}(u)$ and $Z_{s}(u)$ being $\mathcal{F}$-conditionally independent for $s, t \in \mathcal{T}$ with $s \neq t$.

This convergence result is in the space of continuous functions on a compact interval, equipped with the uniform topology. Alternatively, we could have stated a functional convergence result for functions taking values in a weighted $L_{2}$ space. However, since the functions of interest (mainly, $D_{t, \kappa}^{N}$ as functions of $\kappa$ ) are defined on the bounded interval $[0,1]$, it is enough to look at their Fourier transforms only on a compact interval including zero for their analysis. 
We further note that the convergence rate of $\widehat{T D}_{t}^{N}(u)$ is faster than that of $\widehat{D}_{t, \kappa}^{N}$, namely $\sqrt{n}$ versus $\sqrt{k_{n}}$. This is because, in the case of $\widehat{T D}_{t}^{N}(u)$, the errors in measuring dispersion on the entire unit interval are averaged out, which enhances the convergence rate. As a consequence, the requirement on the local window $k_{n}$, i.e., the restriction on $\varrho$, is now much stricter relative to the one needed for Theorem 1 . Nevertheless, $\varrho$ can still take values in a range without impacting the CLT result in Theorem 2, which is a desirable feature for practical applications.

\section{Tests for Cross-Sectional Dispersion in Market Betas}

We now exploit the limit results in the previous section to design tests for hypotheses regarding the beta dispersion. We assume that the limit of $D_{t, \kappa}^{N}$ exists, as the size of the cross-section $N$ converges to $\bar{N}$, for $\bar{N}$ either a finite number or infinity. We denote this limit by,

$$
D_{t, \kappa}=\operatorname{plim}_{N \rightarrow \bar{N}} D_{t, \kappa}^{N}, \quad t \in \mathbb{N}_{+}, \quad \kappa \in[0,1]
$$

and further define,

$$
T D_{t}(u)=\int_{0}^{1} \omega_{u}(\kappa) D_{t, \kappa} d \kappa, \quad t \in \mathbb{N}_{+}, \quad u \in \mathcal{U}
$$

We will apply our theorems by averaging the dispersion measures across trading days. Towards this end, we introduce the notation,

$$
\widehat{D}_{\mathcal{T}, \kappa}^{N}=\sum_{t \in \mathcal{T}} \widehat{D}_{t, \kappa}^{N}, \quad D_{\mathcal{T}, \kappa}^{N}=\sum_{t \in \mathcal{T}} D_{t, \kappa}^{N}, \quad D_{\mathcal{T}, \kappa}=\sum_{t \in \mathcal{T}} D_{t, \kappa},
$$

for $\mathcal{T}$ being a finite set of integers in $[0, T]$. We define similarly $\widehat{T D}_{\mathcal{T}}^{N}(u), T D_{\mathcal{T}}^{N}(u)$, and $T D_{\mathcal{T}}(u)$.

\subsection{Tests for Intraday Variation in Dispersion}

We start by designing tests for determining whether the market beta dispersion varies across the trading day. Specifically, we introduce the set,

$$
\Omega_{\mathcal{T}}\left(\kappa, \kappa^{\prime}\right)=\left\{\omega: D_{\mathcal{T}, \kappa}=D_{\mathcal{T}, \kappa^{\prime}}\right\}, \kappa, \kappa^{\prime} \in[0,1], \kappa \neq \kappa^{\prime}
$$

We then seek to test whether the sample path belongs to $\Omega_{\mathcal{T}}\left(\kappa, \kappa^{\prime}\right)$ or its compliment. For that purpose, we propose a test statistic with the following critical region,

$$
\mathcal{C}_{n}=\left\{\frac{\left|\widehat{D}_{\mathcal{T}, \kappa}^{N}-\widehat{B}_{\mathcal{T}, \kappa}^{N}-\widehat{D}_{\mathcal{T}_{, \kappa^{\prime}}}^{N}+\widehat{B}_{\mathcal{T}, \kappa^{\prime}}^{N}\right|}{\sqrt{\sum_{t \in \mathcal{T}}\left(\widehat{\operatorname{Avar}}\left(\widehat{D}_{t, \kappa}^{N}\right)+\widehat{\operatorname{Avar}}\left(\widehat{D}_{t, \kappa^{\prime}}^{N}\right)\right)}}>z_{1-\alpha / 2}\right\}, \quad \alpha \in(0,1),
$$


where $z_{\alpha}$ is the $\alpha$-quantile of the standard normal distribution. Then, from Theorem 1, we have,

$$
\mathbb{P}\left(\mathcal{C}_{n} \mid \Omega_{\mathcal{T}}\left(\kappa, \kappa^{\prime}\right)\right) \rightarrow \alpha, \quad \mathbb{P}\left(\mathcal{C}_{n} \mid \Omega_{\mathcal{T}}\left(\kappa, \kappa^{\prime}\right)^{c}\right) \rightarrow 1
$$

provided

$$
\left\{\begin{array}{l}
\left(D_{\mathcal{T}, \kappa}^{N}-D_{\mathcal{T}, \kappa}-D_{\mathcal{T}, \kappa^{\prime}}^{N}+D_{\mathcal{T}, \kappa^{\prime}}\right) 1\left(\Omega_{\mathcal{T}}\left(\kappa, \kappa^{\prime}\right)\right)=o_{p}\left(1 / \sqrt{k_{n}}\right) \\
\left(D_{\mathcal{T}, \kappa}^{N}-D_{\mathcal{T}, \kappa}-D_{\mathcal{T}, \kappa^{\prime}}^{N}+D_{\mathcal{T}, \kappa^{\prime}}\right) 1\left(\Omega_{\mathcal{T}}\left(\kappa, \kappa^{\prime}\right)^{c}\right)=o_{p}(1)
\end{array}\right.
$$

The above condition obviously holds, when $\bar{N}$ is finite. When $\bar{N}$ is infinite, the second part of equation (38) typically follows by invoking a Law of Large Numbers for $N \rightarrow \infty$. For the first part of equation (38) with an asymptotically increasing $N$, we require the given rate of convergence for the above-mentioned cross-sectional Law of Large Numbers, and we need $N$ to grow sufficiently fast relative to $k_{n}$. Alternatively, this condition automatically holds without restrictions on the relative size of the two dimensions of the return panel, if the market betas are assumed constant across trading day (recall these assumptions concern only the null hypothesis).

\subsection{Tests for Functional Variation in Dispersion}

We now go on to derive a test for variation in $D_{t, \kappa}$ (as a function of $\kappa$ ) across different trading days. Specifically, we are interested in deciding whether the sample path belongs to the following subset of the sample space,

$$
\Omega\left(\mathcal{T}_{1}, \mathcal{T}_{2}\right)=\left\{\omega: D_{\mathcal{T}_{1}, \kappa}=D_{\mathcal{T}_{2}, \kappa}, \quad \kappa-\text { a.e. }\right\}, \quad \mathcal{T}_{1} \cap \mathcal{T}_{2}=\emptyset
$$

where $\mathcal{T}_{1}$ and $\mathcal{T}_{2}$ are two disjoint sets of integers in $[0, T]$.

As for the previous test, we need a condition for the asymptotic size of the difference $D_{t, \kappa}^{N}-D_{t, \kappa}$ which, in the current setting, takes the form,

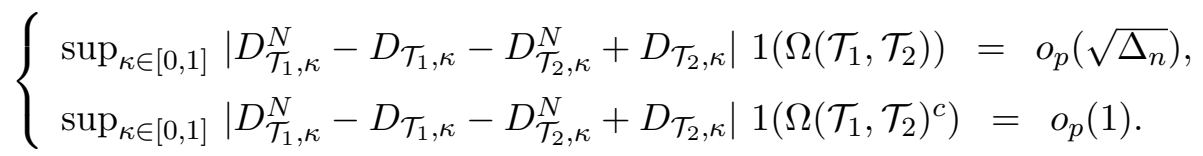

Similar comments to the ones following equation (38) apply here as well. In particular, the first of the above conditions hold, whenever a functional CLT for $D_{t, \kappa}^{N}$, as a function of $\kappa$, with $N \rightarrow \infty$ applies, and $N$ is sufficiently large relative to $n$ (depending on the rate of convergence of this crosssectional CLT). Alternatively, this condition holds without any restriction on $N$, if $\beta_{t+\kappa}^{(j)}=\beta_{s+\kappa}^{(j)}$, for $s, t \in \mathcal{T}, \kappa \in[0,1]$ and $j=1, \ldots, N$.

For constructing the critical region of the test, we exploit the following corollary to Theorem 2. 
Corollary 1. Assume the conditions of Theorem 2 hold for $\mathcal{T}=\mathcal{T}_{1} \cup \mathcal{T}_{2}$, where $\mathcal{T}_{1}$ and $\mathcal{T}_{2}$ are disjoint sets of integers in $[0, T]$. Let $\omega_{u}(z)=e^{i u z}$, for $u \in \mathcal{U}=[0, \overline{\mathcal{U}}]$, and $\overline{\mathcal{U}}$ is a positive constant. (a) If $D_{\mathcal{T}_{1}, \kappa}=D_{\mathcal{T}_{2}, \kappa}$ a.e. for $\kappa \in[0,1]$, and condition (40) holds, then,

$$
\sqrt{n-k_{n}+1} \sup _{u \in \mathcal{U}}\left|\widehat{T D}_{\mathcal{T}_{1}}^{N}(u)-\widehat{T D}_{\mathcal{T}_{2}}^{N}(u)\right| \stackrel{\mathcal{L}-s}{\longrightarrow} \sup _{u \in \mathcal{U}}\left|\sum_{t \in \mathcal{T}_{1}} Z_{t}(u)-\sum_{t \in \mathcal{T}_{2}} Z_{t}(u)\right|,
$$

where the limiting process $Z_{t}(u)$ is defined in Theorem 2.

(b) If $D_{\mathcal{T}_{1}, \kappa} \neq D_{\mathcal{T}_{2}, \kappa}$ for $\kappa$ in a set of positive Lebesgue measure, then,

$$
\sup _{u \in \mathcal{U}}\left|T D_{\mathcal{T}_{1}}(u)-T D_{\mathcal{T}_{2}}(u)\right|>0
$$

Part (a) of the above corollary characterizes the asymptotic behavior of $\widehat{T D}_{\mathcal{T}_{1}}^{N}(u)-\widehat{T D}_{\mathcal{T}_{2}}^{N}(u)$ under the null hypothesis. Part (b) shows that for determining whether $D_{\mathcal{T}_{1}, \kappa}$ differs from $D_{\mathcal{T}_{2}, \kappa}$ (as a function of $\kappa$ ), we only need to look at the difference between their Fourier transforms for $u$ on a compact subset of $\mathbb{R}$ containing zero. This result follows from Bierens (1982); see also Bierens and Ploberger (1997). Given Corollary 1, we propose a test with the following critical region,

$$
\mathcal{C}_{n}=\left\{\sqrt{n-k_{n}+1} \sup _{u \in \mathcal{U}}\left|\widehat{T D}_{\mathcal{T}_{1}}^{N}(u)-\widehat{T D}_{\mathcal{T}_{2}}^{N}(u)\right|>\widehat{z}_{1-\alpha / 2}\right\}, \quad \alpha \in(0,1),
$$

where $\widehat{z}_{\alpha}$ is the $\alpha$-quantile of a centered Gaussian process on $\mathcal{U}$ with covariance and relation func-

tions given by $\sum_{t \in \mathcal{T}_{1} \cup \mathcal{T}_{2}} \widehat{\Sigma}_{t}(u, v)$ and $\sum_{t \in \mathcal{T}_{1} \cup \mathcal{T}_{2}} \widehat{\Xi}_{t}(u, v)$, respectively. We note that $\widehat{z}_{\alpha}$ may be computed easily via simulation. From Corollary 1, it follows, provided condition (40) holds, that,

$$
\mathbb{P}\left(\mathcal{C}_{n} \mid \Omega\left(\mathcal{T}_{1}, \mathcal{T}_{2}\right)\right) \rightarrow \alpha, \quad \mathbb{P}\left(\mathcal{C}_{n} \mid \Omega\left(\mathcal{T}_{1}, \mathcal{T}_{2}\right)^{c}\right) \rightarrow 1
$$

Finally, we observe that a number of suitable substitutions within the above test enables us to test the hypothesis that the market beta dispersion, as a function of the time within the trading day, has a particular parametric specification. Specifically, we must replace $D_{\mathcal{T}_{2}, \kappa}$ by some function

$\bar{D}_{\kappa}$ on $[0,1]$, substitute $\int_{0}^{1} e^{i u \kappa} \bar{D}_{\kappa} d \kappa$ for $\widehat{T D}_{\mathcal{T}_{2}}^{N}(u)$, and set $\mathcal{T}_{2}=\emptyset$, thus obtaining a test for the hypothesis $D_{\mathcal{T}_{1}, \kappa}=\bar{D}_{\kappa}$ for $\kappa \in[0,1]$.

\section{Inference for the Cross-Sectional Distribution of Market Beta}

We finish the theoretical analysis by proposing an estimator for the cross-sectional distribution of market betas and providing the associated limit theory. Hitherto, we have focused on the crosssectional dispersion of market betas. However, as we now show, we can extend this analysis to study the entire cross-sectional beta distribution. We will do this by estimating the characteristic function 
of the cross-sectional beta distribution at a given point in time. The associated convergence result, presented below, is functional and takes place in the complex-valued Hilbert space $\mathcal{L}^{2}(w)$,

$$
\mathcal{L}^{2}(w)=\left\{f:\left.\mathbb{R} \rightarrow \mathbb{C}\left|\int_{\mathbb{R}}\right| f(u)\right|^{2} w(u) d u<\infty\right\}
$$

where $w$ is some positive-valued and continuous weight function with exponential tail decay. The inner product on $\mathcal{L}^{2}(w)$ is induced from the inner products of its real and imaginary parts, i.e., for $f$ and $g$ two elements of $\mathcal{L}^{2}(w)$, we set

$$
\langle f, g\rangle=\int_{\mathbb{R}} f(z) \overline{g(z)} w(z) d z .
$$

Next, for a random complex function $X$ taking values in $\mathcal{L}^{2}(w)$, we introduce the covariance operator

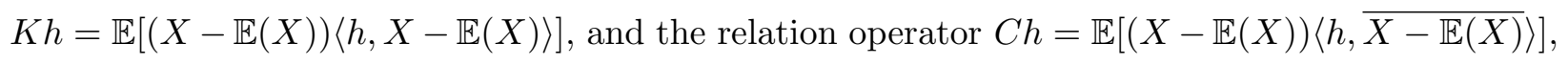
where $h \in \mathcal{L}^{2}(w)$. We recall that a Gaussian law on $\mathcal{L}^{2}(w)$ is uniquely identified by the mean, covariance and relation operators. We refer to this law as $\mathcal{C N}(\mu, K, C)$, for $\mu$ being the mean, $K$ being the covariance, and $C$ being the relation operator.

Our estimator of the characteristic function of the cross-sectional distribution of market beta can be easily constructed from $\widehat{C}_{t, \kappa}^{(j)}(2)$ and $\widehat{V}_{t, \kappa}^{(0, j)}$ in the following manner,

$$
\widehat{\mathcal{L}}_{t, \kappa}^{\beta}(u)=\frac{1}{N} \sum_{j=1}^{N} \exp \left(i u \frac{\widehat{C}_{t, \kappa}^{(j)}(2)}{\widehat{V}_{t, \kappa}^{(0, j)}}+i u\right), \quad u \in \mathbb{R} .
$$

The next theorem provides the CLT for $\widehat{\mathcal{L}}_{t, \kappa}^{\beta}(u)$.

Theorem 3. Suppose Assumptions $A$ and $C$ hold. Let $n \rightarrow \infty$ and $N \rightarrow \bar{N}$, for $\bar{N} \in(0, \infty]$, with $\varpi \in(1 / 4,1 / 2), \varrho \in(0,1 / 2)$ and $\varrho>2-4 \varpi$. Then, for $t \in \mathcal{N}_{+} \cap[0, T]$ and $\kappa \in[0,1]$, we have,

$$
\sqrt{k_{n}}\left(\widehat{\mathcal{L}}_{t, \kappa}^{\beta}(u)-\frac{1}{N} \sum_{j=1}^{N} \exp \left(i u \beta_{t+\kappa}^{(j)}\right)\right) \stackrel{\mathcal{L}-s}{\longrightarrow} \mathcal{Z}_{t, \kappa},
$$

where $\mathcal{Z}_{t, \kappa}$ is $\mathcal{F}$-conditionally $\mathcal{C N}\left(0, K_{t, \kappa}, C_{t, \kappa}\right)$, and the operators $K_{t, \kappa}$ and $C_{t, \kappa}$ are given by,

$$
K_{t, \kappa} h(z)=\int_{\mathbb{R}} k_{t, \kappa}(z, u) h(u) w(u) d u, \quad C_{t, \kappa} h(z)=\int_{\mathbb{R}} c_{t, \kappa}(z, u) h(u) w(u) d u, \quad \forall h \in \mathcal{L}^{2}(w),
$$

with the functions $k_{t, \kappa}(z, u)$ and $c_{t, \kappa}(z, u)$ given in Assumption $C$.

Using feasible version of this result one can make formal inference for the cross-sectional distribution of market betas at a given point in time (or fixed points in time). In particular, we can conduct tests for deciding whether the entire cross-sectional beta distribution changes during a 
trading day or across trading days. In addition, we can estimate the density of the cross-sectional beta distribution at time $t-1+\kappa$, denoted by $f_{t, \kappa}^{\beta}(x)$, via Fourier inversion:

$$
\widehat{f}_{t, \kappa}^{\beta}(x)=\frac{1}{2 \pi} \int_{-u_{n}}^{u_{n}} e^{-i u x} \widehat{\mathcal{L}}_{t, \kappa}^{\beta}(u) d u,
$$

for some positive sequence $u_{n} \rightarrow \infty$ as $n \rightarrow \infty$.

Finally, as for the analysis of the dispersion, we can construct cross-sectional moments of temporally aggregated estimates for betas. Specifically, for $\mathcal{T}$ a set of integers in $[0, T]$, we can define,

$$
\widehat{\mathcal{L}}_{\mathcal{T}, \kappa}^{\beta}(u)=\frac{1}{N} \sum_{j=1}^{N} \exp \left(i u \frac{1}{|\mathcal{T}|} \sum_{t \in \mathcal{T}} \frac{\widehat{C}_{t, \kappa}^{(j)}(2)}{\widehat{V}_{t, \kappa}^{(0, j)}}+i u\right), \widehat{f}_{\mathcal{T}, \kappa}^{\beta}(x)=\frac{1}{2 \pi} \int_{-u_{n}}^{u_{n}} e^{-i u x} \widehat{\mathcal{L}}_{\mathcal{T}, \kappa}^{\beta}(u) d u .
$$

A functional CLT result for $\widehat{\mathcal{L}}_{\mathcal{T}, \kappa}^{\beta}(u)$, analogous to the one in Theorem 3, may be obtained here as well. For brevity, we do not state it explicitly.

\section{Simulation Study}

We now assess the finite sample properties of the new inference procedures. To this end, we conduct a Monte Carlo study in which we vary the sampling frequency, window length, and number of assets in the cross section. The analysis is based on simulation from the following affine jump-diffusion model for the return on the market and each of the $N$ assets,

$$
\begin{aligned}
d X_{t}^{(0)} & =\sqrt{V_{s}} d W_{s}^{(0)}+Z_{t} d N_{t}, \quad V_{t}=V_{t}^{(1)}+V_{t}^{(2)}, \\
d V_{t}^{(i)} & =\kappa_{i}\left(\theta-V_{t}^{(i)}\right)+\xi_{i} \sqrt{V_{t}^{(i)}} d B_{t}^{(i)}, \quad i=1,2, \\
d X_{t}^{(j)} & =\beta_{t}^{(j)} \sqrt{V_{t}} d W_{s}^{(0)}+\sqrt{V_{t}} d \widetilde{W}_{s}^{(j)}+\beta_{t}^{(j)} Z_{t} d N_{t}, \quad j=1, \ldots, N,
\end{aligned}
$$

where $B^{(1)}, B^{(2)}, W^{(0)}, \ldots, W^{(N)}$ are independent standard Brownian motions, $N_{t}$ is a Poisson process with intensity $\lambda_{J}$ capturing the arrival of market jumps with size given by $\left(Z_{s}\right)_{s \geq 1}$, where $Z_{s} \stackrel{\text { i.i.d. }}{\sim} N\left(0, \sigma_{J}^{2}\right)$. The parameters are identical to those in Bollerslev and Todorov (2011),

$$
\left(\kappa_{1}, \kappa_{2}, \theta, \xi_{1}, \xi_{2}, \lambda_{J}, \sigma_{J}^{2}\right)=(0.0128,0.6930,0.4068,0.0954,0.7023,0.2,0.19,0.932) .
$$

Given our objectives, we calibrate the specification for the intraday variation of beta carefully. The market betas obey the following dynamics,

$$
\beta_{t}^{(j)}=\psi_{\lfloor t\rfloor, t-\lfloor t\rfloor}\left(\bar{\beta}^{(j)}\right), \quad j=1, \ldots, N, \quad t \in \mathbb{R}_{+},
$$

where $\bar{\beta}^{(j)}$ is drawn from,

$$
\bar{\beta}^{(j)} \stackrel{\text { i.i.d. }}{\sim} \text { uniform }([0.5,1.5]), \quad j=1, \ldots, N
$$


and the function $\psi$ is given by,

$$
\psi_{t, \kappa}(x)=x+\bar{\psi}_{t, \kappa}(x-1), \quad t \in \mathbb{N}_{+}, \quad \kappa \in[0,1], \quad x \in \mathbb{R},
$$

for some smooth function of $t$ and $\kappa, \bar{\psi}_{t, \kappa}: \mathbb{N}_{+} \times[0,1] \rightarrow \mathbb{R}$. We use three functions, $\left\{\bar{\psi}_{\kappa}^{(i)}\right\}_{i=1,2,3}$, which depend only on $\kappa \in[0,1]$, for $\bar{\psi}_{t, \kappa}$. The first, $\bar{\psi}_{\kappa}^{(1)}$, is a constant, chosen so that if $\bar{\psi}_{t, \kappa}=\bar{\psi}_{\kappa}^{(1)}$, then $\mathbb{E}\left(\beta_{t}^{(j)}-1\right)^{2}$ equals the average daily estimate for the dispersion of the market betas observed in our data. Next, we set $\bar{\psi}_{\kappa}^{(2)}$, so that $\bar{\psi}_{t, \kappa}=\bar{\psi}_{\kappa}^{(2)}$ implies a value for $\mathbb{E}\left(\beta_{t}^{(j)}-1\right)^{2}$ that matches the average dispersion of the market betas as a function of time-of-day in our data. Finally, $\bar{\psi}_{\kappa}^{(3)}$ is calibrated in an analogous way to $\bar{\psi}_{\kappa}^{(2)}$, with the difference being that the implied $\mathbb{E}\left(\beta_{t}^{(j)}-1\right)^{2}$ now matches the average dispersion of the market betas as a function of time-of-day observed in our data only on days with low volatility. For the latter, we find that the beta dispersion as a function of the time-of-day appears to differ substantially from that computed on the other days in the sample. The three functions $\left\{\bar{\psi}_{\kappa}^{(i)}\right\}_{i=1,2,3}$ are displayed in Figure 2.

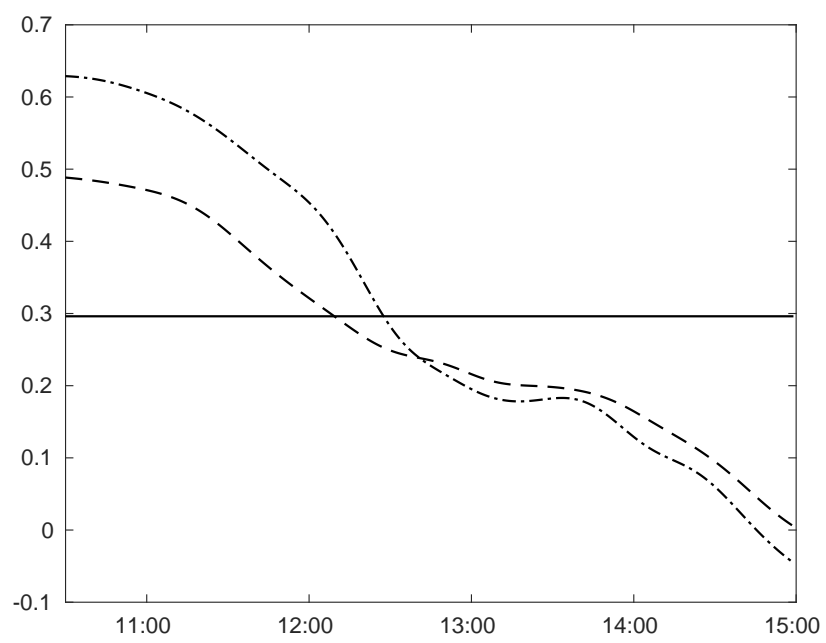

Figure 2: The functions $\left\{\bar{\psi}_{\kappa}^{(i)}\right\}_{i=1,2,3}$ used in the Monte Carlo. The figure plots $\bar{\psi}_{\kappa}^{(1)}$ (solid line), $\bar{\psi}_{\kappa}^{(2)}$ (dashed line) and $\bar{\psi}_{\kappa}^{(3)}$ (dashed-dotted line) used in computing the different beta functions according to equations (54)-(55) in the various Monte Carlo setups detailed in the text.

We fix the various tuning parameters for the statistics with a view towards our empirical application. Throughout, we set $|\mathcal{T}|=65$ or $|\mathcal{T}|=250$ (this applies also to the size of the sets $\mathcal{T}_{1}$ and $\mathcal{T}_{2}$, when performing tests across trading days), corresponding to averaging across a period of one quarter or one year. The truncation level is set at $\nu_{t, n}^{(j)}=4 \sqrt{B V_{t, n}^{(j)}} \Delta_{n}^{0.49}, j=0, \ldots, N$, where $B V_{t, n}^{(j)}$ is the so-called bipower variation of asset $j$, given by $B V_{t, n}^{(j)}=\frac{\pi}{2} \sum_{i=2}^{n}\left|\Delta_{t, i}^{n} X^{(j)}\right|\left|\Delta_{t, i-1}^{n} X^{(j)}\right|$, which is a nonparametric estimate of daily integrated volatility, see Barndorff-Nielsen and Shephard (2004b). Finally, we set $\alpha_{n}=Q_{0.1}(B V) / \log (n)$ (recall equations (12)-(13)), where $Q_{0.1}(B V)$ is the 
10-th quantile of the empirical distribution of $B V^{(0)}$.

We begin by studying the finite-sample properties of the test for equal cross-sectional dispersion in betas during parts of the trading day, given in Section 4.1. The empirical rejection rates of the test under the null hypothesis are provided in Table 1, while those under alternative hypotheses are reported in Table 2. The results in Table 1 point to a satisfactory behavior of the test under the null hypothesis, with the empirical rejection rates being very close to its nominal size. This holds true for all the different values of $n,|\mathcal{T}|$, and $N$ that we consider.

\begin{tabular}{|c|c|c|c|c|c|c|c|}
\hline \multirow[b]{2}{*}{$\mathrm{n}$} & \multirow[b]{2}{*}{$\backslash \mathrm{N}$} & \multicolumn{3}{|c|}{$\alpha=0.05$} & \multicolumn{3}{|c|}{$\alpha=0.1$} \\
\hline & & 100 & 300 & 500 & 100 & 300 & 500 \\
\hline & & \multicolumn{6}{|c|}{$|\mathcal{T}|=65$ days } \\
\hline & 390 & 0.054 & 0.073 & 0.065 & 0.106 & 0.121 & 0.117 \\
\hline & 120 & 0.068 & 0.056 & 0.065 & 0.121 & 0.110 & 0.121 \\
\hline \multirow{2}{*}{\multicolumn{2}{|c|}{78}} & 0.073 & 0.056 & 0.069 & 0.122 & 0.111 & 0.125 \\
\hline & & \multicolumn{6}{|c|}{$|\mathcal{T}|=250$ days } \\
\hline & 390 & 0.058 & 0.062 & 0.053 & 0.115 & 0.115 & 0.107 \\
\hline & 120 & 0.053 & 0.070 & 0.061 & 0.104 & 0.141 & 0.114 \\
\hline & 78 & 0.075 & 0.065 & 0.062 & 0.135 & 0.119 & 0.120 \\
\hline
\end{tabular}

Table 1: Monte Carlo Results: Test for constant beta dispersion across the trading day under the null hypothesis (53)-(55) with $\bar{\psi}_{t, \kappa}=\bar{\psi}_{\kappa}^{(1)}$. The table reports empirical rejection rates of the test in Section 4.1 for nominal size 0.05 and 0.10 , using 1,000 simulations. The time windows for the test are the first and last 2 hours of the trading day.

Turning to our ability to detect market beta intraday variation, Table 2 shows that our test has excellent power properties against the given alternative (we consider equation (55) with $\bar{\psi}_{t, \kappa}=\bar{\psi}_{\kappa}^{(2)}$ as our alternative). This holds true even for the scenario in which we average the dispersion statistic over the smallest of our choices for $|\mathcal{T}|, 65$ trading days. As expected, the power of the test is somewhat lower when comparing the cross-sectional dispersion not at the market open, but rather at lunch, versus the market close. This is because the discrepancy in $D_{t, \kappa}$ between the two points within the trading day now is decidedly smaller, see Figure 2.

Next, we explore the properties of the functional test for variation in dispersion, developed in Section 4.2. We implement it with $\overline{\mathcal{U}}=2 \pi$ and discretize the domain using increments of $\pi / 3$ (i.e., $u \in\{0, \pi / 3,2 \pi / 3, \ldots, 2 \pi\}$ ). The critical test values are obtained from the procedure outlined in 


\begin{tabular}{|c|c|c|c|c|c|c|c|}
\hline \multirow[b]{2}{*}{$\mathrm{n}$} & \multirow[b]{2}{*}{$\backslash \mathrm{N}$} & \multicolumn{3}{|c|}{65 days } & \multicolumn{3}{|c|}{250 days } \\
\hline & & 100 & 300 & 500 & 100 & 300 & 500 \\
\hline & & \multicolumn{6}{|c|}{ open versus close } \\
\hline & 390 & 1.000 & 1.000 & 1.000 & 1.000 & 1.000 & 1.000 \\
\hline & 120 & 1.000 & 1.000 & 1.000 & 1.000 & 1.000 & 1.000 \\
\hline \multirow{2}{*}{\multicolumn{2}{|c|}{78}} & 1.000 & 1.000 & 1.000 & 1.000 & 1.000 & 1.000 \\
\hline & & \multicolumn{6}{|c|}{ lunch versus close } \\
\hline & 390 & 1.000 & 1.000 & 1.000 & 1.000 & 1.000 & 1.000 \\
\hline & 120 & 0.990 & 0.991 & 0.993 & 1.000 & 1.000 & 1.000 \\
\hline & 78 & 0.839 & 0.854 & 0.845 & 1.000 & 1.000 & 1.000 \\
\hline
\end{tabular}

Table 2: Monte Carlo Results: Test for constant beta dispersion across the trading day under the alternative hypothesis (53)-(55) with $\bar{\psi}_{t, \kappa}=\bar{\psi}_{\kappa}^{(2)}$. The table reports empirical rejection rates of the test in Section 4.1 for nominal size 0.05 using 1,000 simulations. The time windows in the test are: open (first two hours), lunch (11am-1pm) and close (last two hours).

Section 4.2 with 10, 000 simulations. Table 3 provides results under the null hypothesis, $\bar{\psi}_{t, \kappa}=\bar{\psi}_{\kappa}^{(2)}$. We notice a slight over-rejection for $T=250$ and low values of $n$. Overall, however, the test has empirical rejections rates under the null hypothesis close to the corresponding nominal size.

To examine the power of the test, we set $\bar{\psi}_{t, \kappa}=\bar{\psi}_{\kappa}^{(2)}$ for $t \in \mathcal{T}_{1}$ and let $\bar{\psi}_{t, \kappa}=\bar{\psi}_{\kappa}^{(3)}$ for $t \in \mathcal{T}_{2}$. Results for this simulation scenario are given in Table 4 and reveal good power of our test. Not surprisingly, the power improves as the sampling frequency increases. We further note that the rejection rates appear insensitive to the choice of $N$ (the size of the cross-section).

\section{$7 \quad$ Empirical Study of Intraday Market Beta Dispersion}

We now use the developed econometric tools to study empirically the intraday behavior of the market betas. The analysis is based on high-frequency returns on the constituents of the S\&P 500 stock market index over the period 2010-2018. We only retain stocks that belong to the index for the entire sample period, leaving us with a cross-section of 329 stocks. Our proxy for the market is the SPY ETF on the S\&P 500 index. On each trading day, we sample the asset prices at the 3-minute frequency. This provides us with 120 return observations per day for each asset. We remove days with partial trading. Overall, the sample contains 2245 full trading days. 


\begin{tabular}{|c|c|c|c|c|c|c|c|}
\hline \multirow[b]{2}{*}{$\mathrm{n}$} & \multirow[b]{2}{*}{$\backslash \mathrm{N}$} & \multicolumn{3}{|c|}{$\alpha=0.05$} & \multicolumn{3}{|c|}{$\alpha=0.1$} \\
\hline & & 100 & 300 & 500 & 100 & 300 & 500 \\
\hline & & \multicolumn{6}{|c|}{$\bar{\psi}_{t, \kappa}=\bar{\psi}_{t, \kappa}^{(2)}, T=65$} \\
\hline & 390 & 0.048 & 0.055 & 0.055 & 0.103 & 0.112 & 0.104 \\
\hline & 120 & 0.051 & 0.056 & 0.055 & 0.104 & 0.102 & 0.100 \\
\hline \multirow{2}{*}{\multicolumn{2}{|c|}{78}} & 0.044 & 0.060 & 0.068 & 0.100 & 0.111 & 0.109 \\
\hline & & \multicolumn{6}{|c|}{$\bar{\psi}_{t, \kappa}=\bar{\psi}_{t, \kappa}^{(2)}, T=250$} \\
\hline & 390 & 0.066 & 0.063 & 0.056 & 0.116 & 0.117 & 0.111 \\
\hline & 120 & 0.065 & 0.082 & 0.070 & 0.122 & 0.147 & 0.132 \\
\hline & 78 & 0.074 & 0.070 & 0.055 & 0.127 & 0.131 & 0.118 \\
\hline
\end{tabular}

Table 3: Monte Carlo Results: Test for constant dispersion of betas across time under the null hypothesis (53)-(55) with $\bar{\psi}_{t, \kappa}=\bar{\psi}_{t, \kappa}^{(2)}$. The table reports empirical rejection rates of the test in Section 4.2 of nominal size 0.05 (first three columns) and 0.1 (last three columns) using 1,000 simulations.

\begin{tabular}{|c|c|c|c|c|c|c|c|}
\hline \multirow[b]{2}{*}{$\mathrm{n}$} & \multirow[b]{2}{*}{1} & \multicolumn{3}{|c|}{65 days } & \multicolumn{3}{|c|}{250 days } \\
\hline & & 100 & 300 & 500 & 100 & 300 & 500 \\
\hline & 390 & 1.000 & 1.000 & 1.000 & 1.000 & 1.000 & 1.000 \\
\hline & 120 & 0.889 & 0.878 & 0.873 & 1.000 & 1.000 & 1.000 \\
\hline & 78 & 0.601 & 0.593 & 0.579 & 0.991 & 0.989 & 0.993 \\
\hline
\end{tabular}

Table 4: Monte Carlo Results: Test for constant beta dispersion over time under the alternative hypothesis. The table reports empirical rejection rates of the test of Section 4.2 for nominal size 0.05 using 1,000 simulations. The testing is based on two blocks with $\bar{\psi}_{t, \kappa}=\bar{\psi}_{\kappa}^{(2)}$ for $t \leq 65(t \leq 250)$ and $\bar{\psi}_{t, \kappa}=\bar{\psi}_{\kappa}^{(3)}$ for $t>65(t>250)$ for the first (last) three columns.

\subsection{Empirical Evidence}

First, we compute the average dispersion measure for the entire sample, $\frac{1}{T} \sum_{t=1}^{T} \widehat{D}_{t, \kappa}^{N}$. The function is plotted in the left panel of Figure 3. It reveals that the cross-sectional dispersion of the market betas declines monotonically over the trading day. The reduction is substantial: $\frac{1}{T} \sum_{t=1}^{T} \widehat{D}_{t, \kappa}^{N}$ at the market close is less than half of its value at the open. This is consistent with the illustrative plot 
for the two stocks displayed in Figure 1 of the Introduction. We can now test whether the crosssectional dispersion in market betas is invariant across the trading day by applying the procedures developed in Section 4.1. A natural concern is the potential for a confounding effect from excessive noise in the beta estimates at the start of trading due to high idiosyncratic volatility at the market open. The formal test in Section 4.1 accounts for such features, and thus can discriminate between this type of explanation relative to true changes in the distribution of market betas. Our tests comparing the dispersion over various parts of the trading day overwhelmingly reject the null hypothesis, with p-values equalling zero up the fourth digit after the decimal point.

The evidence from the left panel of Figure 3 suggests that high beta stocks (in excess of unity) have their market beta decline during the trading day, while the opposite is true for the low beta stocks. This conjecture is confirmed in the right panel of Figure 3. It plots the cross-sectional quantiles of the market betas across the trading day. The changes for the two extreme quantiles (the 10'th and 90'th) are most significant, while the median market beta displays little variation over the trading day. To explore whether this intraday pattern in market betas is robust, we repeated the analysis for 2-year subsamples. The message of a declining cross-sectional dispersion in market betas over the trading day remains intact, with the evidence strengthening in the second half of the sample. For brevity, we do not report these results here.
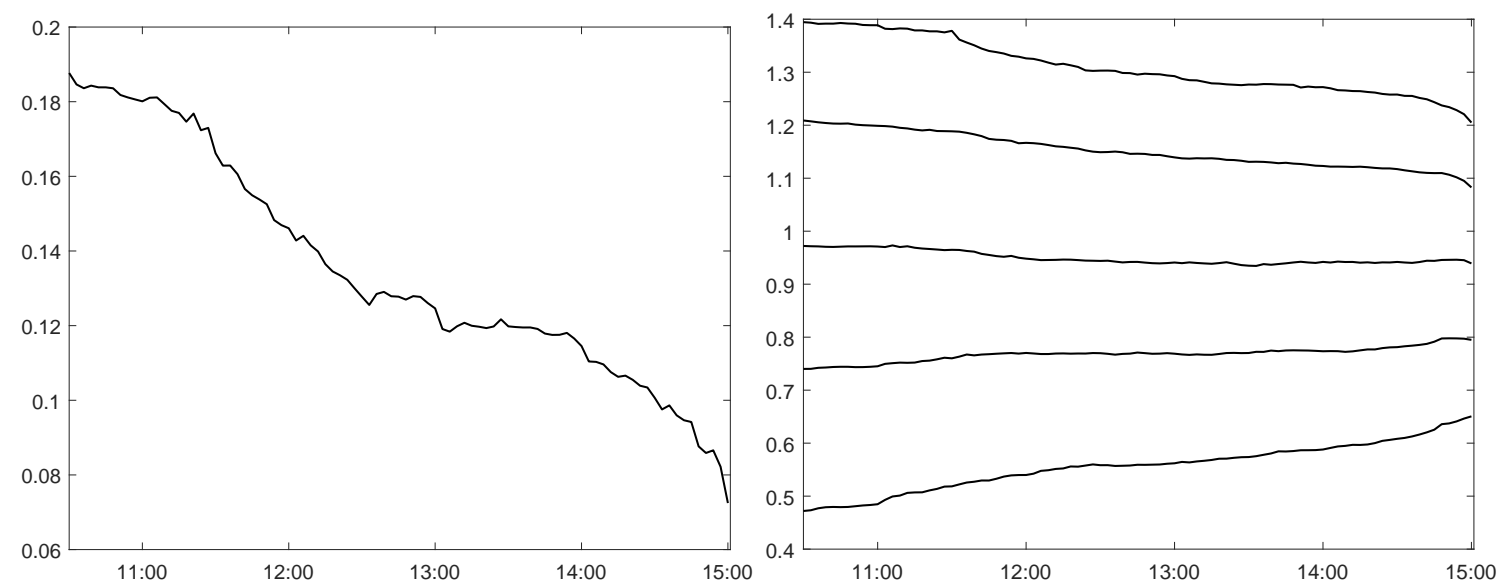

Figure 3: Cross-Sectional Distribution of Market Betas across the Trading Day. The left panel displays the cross-sectional dispersion in market betas and the right panel plots the corresponding quantiles. All quantities are treated as functions of the trading day and computed by averaging over the entire sample. The selected quantiles are: 10th, 25th, 50th, 75th, and 90th.

Using the analysis of Section 5, Figure 4 depicts our estimates of the cross-sectional distribution of the market betas at the open and close of trading. They are clearly very different, with the former having a significantly wider support than the latter, even if they both have modes close to unity. 


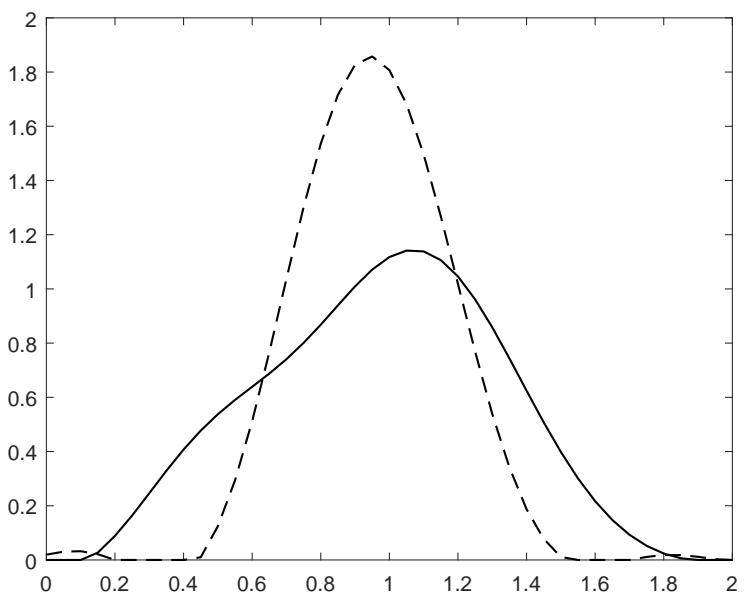

Figure 4: Cross-Sectional Distribution of Market Betas at Open and Close of Trading. The plot displays $\widehat{f}_{\mathcal{T}, \kappa}^{\beta}(x)$ using the first two hours of trading (solid line) and the last two hours of trading (dashed line), both computed over the entire sample. The tuning parameter $u_{n}$ in the Fourier inversion is set to $\inf \left\{0<u:\left|\mathcal{L}_{\mathcal{T}, \kappa}^{\beta}(u)\right|<0.0005\right\}$.

Given the overwhelming evidence for intraday variation in betas, we now explore the evolution of this pattern over time. Figure 5 plots the time-series for the cross-sectional dispersions of betas at the market open and close, representing the parts of the active trading day with the highest and lowest dispersion. To mitigate the impact of estimation error, we report dispersion measures computed over rolling window of 250 days. The dispersion of the market open betas fluctuates greatly across time, unlike that of the market close betas. In fact, following a decline towards the end of 2013, the beta dispersion at market close has been remarkably stable. In contrast, the dispersion at the market open increases initially till some time in 2012, and then gradually declines, reaching a low during 2015, when the gap between the dispersion of the market open and close betas also is the smallest in our sample. Since then, the dispersion in the market open betas increases sharply, about four-fold, and remains highly elevated for the remainder of our sample.

Overall, Figure 5 strongly suggests that the intraday pattern of market betas is evolving across our sample period. We may explore this hypothesis through the formal test procedures developed in Section 4.2. The results are reported in Table 5. We perform the test annually, i.e., we test whether the cross-sectional dispersion of betas, as a function of time-of-day, changes across the calendar years in our sample. The reported results show that, for most calendar year pairings, the null hypothesis of an equal intraday pattern for the cross-sectional dispersion of market betas is overwhelmingly rejected. Interestingly, the last three years of our sample, when the cross-sectional dispersion of market betas at the open of trading has increased significantly, the intraday dispersion pattern appears more stable, or similar, across adjacent years. 


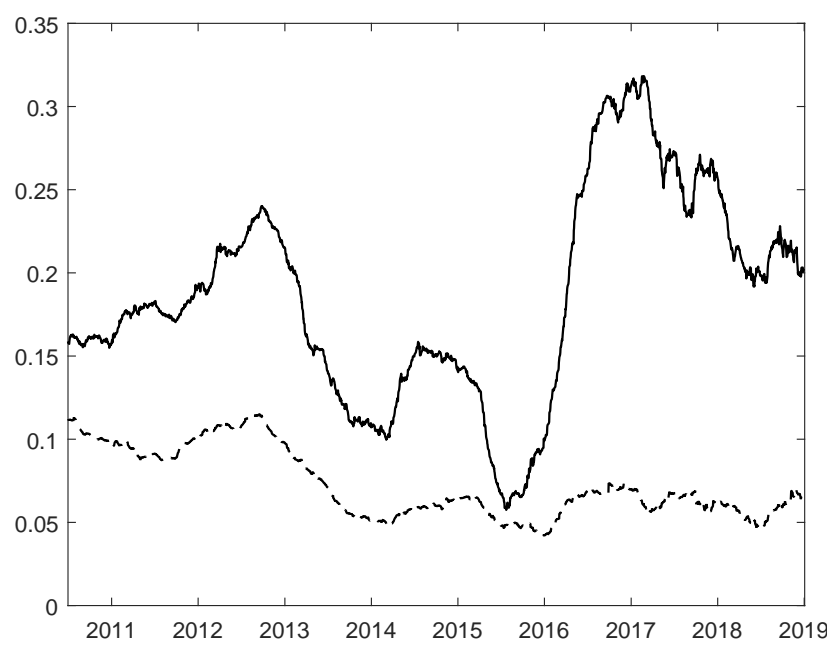

Figure 5: Cross-Sectional Distribution of Market Betas over Time. The figure displays the cross-sectional dispersion of market betas at open (solid line) and close (dashed line) over the full sample. Each dispersion measures is computed using a rolling window of 250 trading days.

\begin{tabular}{lllllllll}
\hline & 2011 & 2012 & 2013 & 2014 & 2015 & 2016 & 2017 & 2018 \\
\hline 2010 & 0.010 & 0.000 & 0.000 & 0.005 & 0.000 & 0.000 & 0.001 & 0.033 \\
2011 & & 0.058 & 0.000 & 0.000 & 0.000 & 0.000 & 0.009 & 0.069 \\
2012 & & & 0.000 & 0.000 & 0.000 & 0.002 & 0.063 & 0.004 \\
2013 & & & 0.001 & 0.003 & 0.000 & 0.000 & 0.000 \\
2014 & & & & 0.000 & 0.000 & 0.000 & 0.004 \\
2015 & & & & & 0.000 & 0.000 & 0.000 \\
2016 & & & & & & & 0.540 & 0.002 \\
2017 & & & & & & & & \\
\hline \hline
\end{tabular}

Table 5: Tests for Changes in Cross-Sectional Dispersion of Market Betas over Time. The table reports p-values for tests of equal dispersion of market betas as functions of time-ofday. Each entry corresponds to a pairwise test, detailed in Section 4.2, involving the years in the corresponding rows and columns.

It is natural to ask whether the time variation in the cross-sectional beta dispersion, as a function of the time within the trading day, can be explained by observable economic variables. We begin to explore this issue by investigating the dependence of $D_{t, \kappa}$ (as a function of $\kappa$ ) on the level of market volatility. To the extent that the intraday variation in market betas is associated with the information flow and the separation of different type of trading (e.g., based on private signals or 
hedging motives) across the trading day, the level of market volatility should affect the intraday evolution of the betas. As a proxy for the market volatility, we use the level of the VIX volatility index, computed from the CBOE option exchange, at the beginning of the trading day. Using the time series empirical quantiles of the VIX series at market open over our sample period, we split the sample into low, medium and high volatility regimes according to the level of VIX falling into the 5th-to-25th quantile, the 40th-to-60th quantile, and the 75th-to-95th quantile, respectively. We then compute our beta dispersion measures in each of the three volatility regimes and display the results in the left panel of Figure 6. Interestingly, the figure reveals no big discrepancies in the compression of the cross-sectional dispersion of betas in the afternoon across the three volatility regimes. The main distinction is that the elevation of $D_{t, \kappa}$ is more pronounced in the low volatility regime than in the other regimes at the beginning of the trading day.
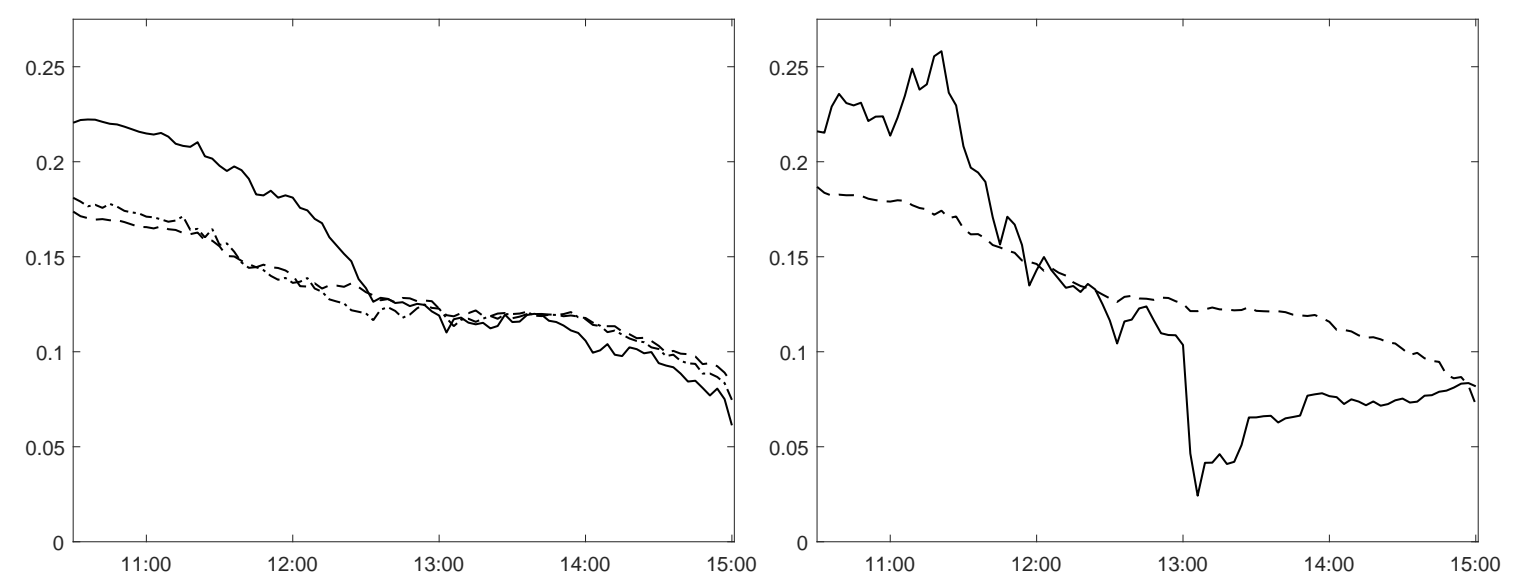

Figure 6: Variation in Cross-Sectional Distribution of Market Betas. The left panel displays the cross-sectional dispersion in the market betas computed on days of low (solid line), medium (dot-dashed line), and high volatility (dashed line). The low, medium, and (high) volatility regimes are defined by the market open VIX falling within its 5th-to-25th, 40th-to-60th, and 75thto-95th empirical quantiles. The right panel depicts the dispersion of the market betas computed on days with (solid line) and without (dashed line) FOMC announcements.

Using the results of Section 4.2, we now formally test the null hypothesis of no difference in the beta dispersion function across the volatility regimes. The results are reported in Table 6 and are in line with the pattern in the left panel of Figure 6. In particular, there is no significant difference in the intraday behavior of the betas in the high and medium volatility regimes. However, there is evidence of significant discrepancies in the intraday beta behavior between the low volatility regime and the other regimes, with our test rejecting the null hypothesis at the $1 \%$ significance level. As Figure 6 indicates, this is primarily due to the behavior of the market betas in the first few hours after the market open during the low volatility regime. 


\begin{tabular}{cc}
\hline Test Pair & P-value \\
\hline Low vs. Medium Volatility & 0.019 \\
Low vs. High Volatility & 0.005 \\
Medium vs. High Volatility & 0.938 \\
\hline \hline
\end{tabular}

Table 6: Cross-Sectional Dispersion in Market Betas Across Volatility Regimes. The table reports p-values of the test in Section 4.2 for equal cross-sectional beta dispersion as a function of time-of-day across volatility regimes. The volatility regimes are defined in Figure 6.

We finally compare the beta dispersion functions on days with and without pre-scheduled FOMC announcements. The release of information regarding the FOMC meeting usually occurs at 1pm Central Time during our sample period. The estimated dispersion functions are displayed in the right panel of Figure 6. Due to the relatively low number of days with FOMC announcements in our sample (a total of 69), the estimated beta dispersion function for these days is visibly noisier. Nevertheless, the difference relative to the beta dispersion function on days without FOMC announcement is evident. The beta dispersion function is somewhat steeper on days with FOMC announcement and there is a sharp drop in the beta dispersion right around the announcement time, and it then remains flat afterwards for the remainder of the trading day. The formal test for equal dispersion functions on days with and without FOMC announcements is in line with the above discussion. It rejects the hypothesis with p-value well below conventional significance levels. At an intuitive level, our results suggest that the stocks become more similar in terms of their exposure to systematic macro level risk immediately following the FOMC meeting. ${ }^{1}$

Overall, our empirical results document significant intraday variation in the market betas across the trading day, with systematic risk in stocks being more dispersed at the start of trading and becoming more concentrated towards the market close and following the release of macroeconomic news of relevance for the entire economy, as exemplified through the FOMC announcements. Furthermore, the market beta dispersion function, viewed as a function of the time within the trading day, varies substantially across the sample, with a significant part of this variation stemming from the behavior of the betas at the market open.

\footnotetext{
${ }^{1}$ One may conjecture that different types of news with economy-wide implications will have a differential shortterm impact on the cross-sectional dispersion of market betas. Some economic developments may favor specific economic sectors relative to others, while updates regarding policy changes intended to fuel or dampen the level of general economic activity may be associated with a more uniform market dependency across all equities. Intuitively, the FOMC announcements would fall in the latter group, implying a narrowing of the beta distribution. Further pursuit of such ideas is outside the scope of the present paper.
} 


\subsection{Asset Pricing Implications}

We now explore the asset pricing implications of our empirical results, indicating systematic intraday changes in the cross-sectional distribution of market betas. Many asset pricing models, including the Capital Asset Pricing Model (CAPM), stipulate that the return on the market portfolio is a component of the aggregate pricing kernel. Therefore, if the persistent changes in the market betas over the trading days matter for asset pricing, we should expect to find a statistically significant and time-varying return on portfolios that have a positive exposure to the market, simply due to this intraday variation in betas. We test this hypothesis now. Specifically, each day at the start of trading, we sort stocks according to their market open beta. This beta is computed at the beginning of each month and kept constant during the rest of the month, using high-frequency returns from the first two hours of trading on the preceding 250 trading days. Our portfolio takes a long position in the bottom $1 / 3$ of stocks sorted on the market open beta and a short position in the corresponding top $1 / 3$ group of stocks. We adjust the weight on the long position, so that the market beta on our constructed portfolio is exactly zero at the open of trading, i.e., the portfolio is market-neutral. We hold the position throughout the trading day and close it at the end of trading. We then open the position in the corresponding portfolio again the following trading day and hold it until the close, and repeat this procedure every trading day in our sample. Given the documented intraday pattern in market betas, i.e., they converge towards unity during the trading day, our initial market-neutral position at the open will gradually build a positive market exposure over the course of the trading day, reaching a maximum at the close. Therefore, if the intraday compression of beta towards its cross-sectional mean is associated with a risk premium, we should expect the above portfolio to earn excess profits. The findings from this exercise are summarized in Table 7. They corroborate the risk premium conjecture as, indeed, the portfolio return is positive, and both economically and statistically significant.

In the above strategy, we did not hold stocks overnight due to the absence of a time-of-day estimate for the market betas during the market close-to-open period. However, it is evident that there is a transition overnight from a low to high beta dispersion. Therefore, it is natural to check whether this systematic overnight variation in betas is also priced. For that purpose, we now sort the stocks at the end of each trading day according to the market close beta. The long and short positions are now switched: the portfolio will be long high market-close beta stocks and short low market-close beta stocks. The portfolio is held only for the close-to-open period. The portfolio is constructed to be market-neutral at the end of the trading day, but will have positive overnight exposure to the market. Hence, if this variation in beta is priced, we also expect this portfolio 


\begin{tabular}{cccc}
\hline \multirow{2}{*}{ Sorting Variable } & \multicolumn{3}{c}{ Return on Strategy } \\
& Intraday & Overnight & Daily \\
\hline Market Open Beta & 1.43 & -0.58 & 0.89 \\
& $(2.56)$ & $(-3.05)$ & $(1.53)$ \\
Market Close Beta & -0.69 & 0.38 & -0.31 \\
& $(-2.83)$ & $(5.03)$ & $(-1.18)$ \\
\hline
\end{tabular}

Table 7: Expected Returns on Time-of-Day Variation in Market Betas. The table reports monthly percentage returns on strategies based on market beta sorts at market open or close. The portfolios consist of stocks in the bottom and top $33 \%$ of the cross-sectional beta distribution. The portfolio positions are adjusted to be market neutral at formation. The portfolio is short/long high beta stocks and long/short low beta stocks, when sorting on open/close betas, and the portfolio is rebalanced each trading day. The last three columns correspond to the part of the day over which the position is held. The betas are computed over windows of length 250 days, using the first and last two hours of trading. Robust t-statistics are reported in parenthesis.

to earn a positive premium. In fact, Table 7 confirms this hypothesis. The mean return of the portfolio is lower than for the one formed over the trading day, but the overnight portfolio has a much smaller variance, leading to an even higher degree of statistical significance.

It follows that, if we were to hold the portfolio formed on the basis of sorting at the market open only during the overnight period, then it should earn a negative risk premium. Likewise, our portfolio formed from a sort on the market-close betas should lose money, if held exclusively during the trading day. These predictions are consistent with the evidence reported in Table 7 . Consequently, if either of the two portfolios (sorted on the open- and close-market betas) is held for the entire day-trading hours plus overnight-its systematic return components will cancel, leaving an overall small and insignificant return. This testifies to the high-frequency nature of the portfolio premium, which sets it apart from a corresponding literature exploiting beta anomalies to design profitable trading strategies. For example, our intraday portfolio, that involves shorting high beta stocks and taking long positions in low beta stocks, is similar to the "betting against beta" strategy proposed by Frazzini and Pedersen (2014) to exploit the empirical finding that high beta stocks earn lower risk premium than implied by the CAPM; see, e.g., Black (1972) and Black, Jensen, and Scholes (1972). However, Table 7 shows that our intraday "betting-against-beta" portfolio does not earn a statistically significant return, when held during the entire day (including the overnight period). Thus, the risk premium, we identify for our intraday and overnight portfolios, is fundamentally different from the "beta anomaly" exploited in Frazzini and Pedersen (2014). In 
fact, the latter is associated mostly with small stocks, see, e.g., Novy-Marx and Velikov (2018), and those stocks are excluded from our analysis. In contrast, our explanation for the presence of a risk premium is that, by construction, the portfolio has a positive market exposure within the trading day, so the compensation is "rational" - stemming from the systematic time-variation of the intraday market betas.

To further check the robustness of our result concerning the risk premium associated with the intraday (and overnight) variation in market betas, we next explore whether it can be rationalized through exposure to well-known market-neutral risk factors. Indeed, a potential alternative explanation for the evidence in Table 7 is that the excess returns reflects compensation for exposure to other systematic sources of priced risk. Primary candidates are the size and value factors of Fama and French (1996), as well as the momentum factor introduced by Jegadeesh and Titman (1993) and Carhart (1997). There is an extensive literature in empirical finance documenting the ability of these factors to explain the cross-sectional behavior of expected stock returns, in addition to the market factor. We use daily data on the Fama and French and momentum factors, using a rolling window of 250 days to compute the loadings of the various portfolios, whose excess returns are reported in Table 7. Even after controlling for this exposure of our intraday and overnight long-short portfolios, the excess returns remain nearly unchanged, leaving the statistical significance intact. This reflects the fact that our portfolios, capturing the intraday and overnight market beta variation, have a very weak association with the size, value and momentum risk factors. For brevity, we do not report these results, but they are available upon request.

Finally, the evidence in Table 7 regarding the differential behavior of the constructed portfolios is reminiscent of empirical results reported by Lou, Polk, and Skouras (2019), as they document opposing excess returns for known trading strategies during the trading day versus overnight. However, the approach and conclusions are fundamentally different. Lou, Polk, and Skouras (2019) do not explore high-frequency fluctuations in the risk exposures, and they ascribe their findings to a reversal of the risk premium across segments of the (calendar) day. In contrast, our evidence is consistent with a reward for market risk exposure, both intraday and overnight. Hence, while Lou, Polk, and Skouras (2019) end up explaining the observed patterns through clientele effects, we "rationalize" the different behavior of the constructed portfolios directly through the documented intraday variation in market betas. 


\section{Conclusion}

This paper explores the intraday variation in market betas in a general nonparametric setting. The inference is based on a cross-section of stocks and the market portfolio. The time span of the return panel is fixed, while the sampling frequency increases to infinity. The size of the crosssection is either fixed or increases asymptotically. We form estimates of the cross-sectional market beta dispersion over local time windows. We derive a feasible limit theory for the beta dispersion measures, both for a fixed number of distinct times during the trading day and in a functional sense. We further extend the analysis by developing a functional limit theory for estimates of the characteristic function of the cross-sectional beta distribution at given points in time. Exploiting these econometric tools, we find strong evidence for systematic variation in the cross-sectional dispersion in market betas, both during the trading day and across trading days. Market betas have the highest dispersion at the market open and tend to compress towards unity gradually during the trading day. We further document that portfolios designed to generate an increasing net market exposure over the course of the trading day earn substantial risk premiums, consistent with the intraday pattern in market betas constituting a source of priced risk.

\section{Assumptions and Proofs}

In the proofs, we denote with $K$ a positive constant that does not depend on $n$ and $N$, and can change from one line to another.

\subsection{Assumptions}

Assumption A. For the processes $\left(X^{(j)}\right)_{j \geq 0}$ we have:

(a) For a sequence of stopping times, $\left(T_{m}\right)_{m \geq 1}$, increasing to infinity, the processes $\left(\alpha^{(j)}\right)_{j \geq 0}$, $\left(\beta^{(j)}\right)_{j \geq 1},\left(\gamma^{(j)}\right)_{j \geq 1}$, and $\left(\widetilde{\sigma}^{(j)}\right)_{j \geq 1}$, are all uniformly bounded on $\left[0, T \wedge T_{m}\right]$.

(b) The processes $\left|\sigma_{t}^{(0)}\right|$ and $\left|\sigma_{t-}^{(0)}\right|$ take positive values on $[0, T]$.

(c) For a sequence of stopping times, $\left(T_{m}\right)_{m \geq 1}$, increasing to infinity and a sequence of constants, $\left(K_{m}\right)_{m \geq 1}$, we have uniformly in $j \geq 1$ :

$$
\begin{gathered}
\mathbb{E}\left[\sup _{s, t \in\left[0, T \wedge T_{m}\right]}\left|\sigma_{t}^{(0)}-\sigma_{s}^{(0)}\right|^{2}+\sup _{s, t \in\left[0, T \wedge T_{m}\right]}\left|\beta_{t}^{(j)}-\beta_{s}^{(j)}\right|^{2}+\sup _{s, t \in\left[0, T \wedge T_{m}\right]}\left|\widetilde{\sigma}_{t}^{(j)}-\widetilde{\sigma}_{s}^{(j)}\right|^{2}\right. \\
\left.+\sup _{s, t \in\left[0, T \wedge T_{m}\right]}\left|\gamma_{t}^{(j)}-\gamma_{s}^{(j)}\right|\left|\gamma_{t}^{(j)}-\gamma_{s}^{(j)}\right|^{\top}\right] \leq K_{m}|t-s|, \\
\left|\mathbb{E}\left(\beta_{t \wedge T_{m}}^{(j)}-\beta_{s \wedge T_{m}}^{(j)}\right)\right|+\left|\mathbb{E}\left(\sigma_{t \wedge T_{m}}^{(0)}-\sigma_{s \wedge T_{m}}^{(0)}\right)\right| \leq K_{m}|t-s|,
\end{gathered}
$$




$$
\left|\mathbb{E}\left(\chi_{s \wedge T_{m}, t \wedge T_{m}}^{(1)} \chi_{s \wedge T_{m}, t \wedge T_{m}}^{(2)}\right)\right| \leq K_{m}|t-s|^{2},
$$

for $\chi_{s, t}^{(1)}$ equal to $\beta_{t}^{(j)}-\beta_{s}^{(j)}$ or $\sigma_{t}^{(0)}-\sigma_{s}^{(0)}$, and $\chi_{s, t}^{(2)}$ equal to one of $\left(W_{t}^{(0)}-W_{s}^{(0)}\right)^{2}-(t-s)$, $\left(W_{t}^{(0)}-W_{s}^{(0)}\right)\left(B_{t}-B_{s}\right)$ and $\left(W_{t}^{(0)}-W_{s}^{(0)}\right)\left(\widetilde{W}_{t}^{(j)}-\widetilde{W}_{s}^{(j)}\right)$.

(d) We have $\sum_{s \leq t} \Delta X_{s}^{(j)}=\int_{0}^{t} \int_{E} \delta^{(j)}(s, u) \mu(d s, d u)$, for $j \geq 0$, and where $\mu$ is a Poisson random measure on $\mathbb{R}_{+} \times E$ with compensator $d s \otimes \nu(d u)$, for some $\sigma$-finite measure $\nu$ on a Polish space $E$. Furthermore, the jump size functions $\delta^{(j)}$ are mappings $\Omega \times \mathbb{R}_{+} \times E \rightarrow \mathbb{R}$ which are locally predictable. For $j=0,1, \ldots$, we have $\int_{0}^{T} \int_{E} 1\left(\delta^{(j)}(s, u) \neq 0\right) d s \nu(d u)<\infty$. For a sequence of stopping times, $\left(T_{m}\right)_{m \geq 1}$, increasing to infinity and a sequence of nonnegative functions $\left(\Gamma_{m}(u)\right)_{m \geq 1}$ satisfying $\int_{E}\left(1 \vee \Gamma_{m}^{2}(u)\right) \nu(d u)<\infty$, we have $\left|\delta^{(j)}(s, u)\right| \leq \Gamma_{m}(u)$, uniformly in $j \geq 0$, for $s \in\left[0, T \wedge T_{m}\right]$.

Assumption B. We have the following uniform convergence in probability as $N \rightarrow \bar{N}$ for some $\bar{N} \in(0, \infty]:$

$$
\begin{aligned}
& \sup _{t=1, \ldots, T, \kappa \in[0,1]}\left|\frac{6}{N} \sum_{j=1}^{N}\left[\left(\beta_{t-1+\kappa}^{(j)}-1\right)^{2} \frac{\left(\widetilde{\sigma}_{t-1+\kappa}^{(j)}\right)^{2}}{\left(\sigma_{t-1+\kappa}^{(0)}\right)^{2}}\right]-\psi_{t, \kappa}^{(a)}\right| \stackrel{\mathbb{P}}{\longrightarrow} 0, \\
& \sup _{t=1, \ldots, T, \kappa \in[0,1]}\left|4\left(\frac{1}{N} \sum_{j=1}^{N}\left(\beta_{t-1+\kappa}^{(j)}-1\right)^{2}\right)^{2}-\psi_{t, \kappa}^{(b)}\right| \stackrel{\mathbb{P}}{\longrightarrow} 0, \\
& \sup _{t=1, \ldots, T, \kappa \in[0,1]}|| \frac{6}{N} \sum_{j=1}^{N}\left[\left(\beta_{t-1+\kappa}^{(j)}-1\right) \frac{\gamma_{t-1+\kappa}^{(j)}}{\sigma_{t-1+\kappa}^{(0)}}\right]-\Lambda_{t, \kappa} \| \stackrel{\mathbb{P}}{\longrightarrow} 0,
\end{aligned}
$$

for some $\psi_{t, \kappa}^{(a)}, \psi_{t, \kappa}^{(b)}$ and $\Lambda_{t, \kappa}$, which are càdlàg functions of $t-1+\kappa$, and $\|\cdot\|$ denoting the Frobenius norm of a matrix. We further set $\psi_{t, \kappa}^{(c)}=\Lambda_{t, \kappa} \Lambda_{t, \kappa}^{\top}$.

To state the next assumption, we introduce the following notation:

$$
\begin{aligned}
& k_{t, \kappa}^{N}(z, u)=-\frac{1}{N} \sum_{j=1}^{N} e^{i(u+z) \beta_{t-1+\kappa}^{(j)} u z} {\left[\left(\beta_{t-1+\kappa}^{(j)}-1\right)^{4}+\frac{3}{2}\left(\beta_{t-1+\kappa}^{(j)}-1\right)^{2} \frac{\gamma_{t-1+\kappa}^{(j)}\left(\gamma_{t-1+\kappa}^{(j)}\right)^{\top}}{\left(\sigma_{t-1+\kappa}^{(0)}\right)^{2}}\right.} \\
&\left.+\frac{3}{2}\left(\beta_{t-1+\kappa}^{(j)}-1\right)^{2} \frac{\left(\widetilde{\sigma}_{t-1+\kappa}^{(j)}\right)^{2}}{\left(\sigma_{t-1+\kappa}^{(0)}\right)^{2}}\right], \\
& c_{t, \kappa}^{N}(z, u)=\frac{1}{N} \sum_{j=1}^{N} e^{i(z-u) \beta_{t-1+\kappa}^{(j)} u z}\left[\left(\beta_{t-1+\kappa}^{(j)}-1\right)^{4}+\frac{3}{2}\left(\beta_{t-1+\kappa}^{(j)}-1\right)^{2} \frac{\gamma_{t-1+\kappa}^{(j)}\left(\gamma_{t-1+\kappa}^{(j)}\right)^{\top}}{\left(\sigma_{t-1+\kappa}^{(0)}\right)^{2}}\right. \\
&\left.+\frac{3}{2}\left(\beta_{t-1+\kappa}^{(j)}-1\right)^{2} \frac{\left(\widetilde{\sigma}_{t-1+\kappa}^{(j)}\right)^{2}}{\left(\sigma_{t-1+\kappa}^{(0)}\right)^{2}}\right] .
\end{aligned}
$$


Assumption C. We have the following convergence in probability as $N \rightarrow \bar{N}$, with some $\bar{N} \in$ $(0, \infty]$ :

$$
\begin{aligned}
& \int_{\mathbb{R}} \int_{\mathbb{R}}\left|k_{t, \kappa}^{N}(z, u)-k_{t, \kappa}(z, u)\right|^{2} w(u) w(z) d u d z \stackrel{\mathbb{P}}{\longrightarrow} 0, \\
& \int_{\mathbb{R}} \int_{\mathbb{R}}\left|c_{t, \kappa}^{N}(z, u)-c_{t, \kappa}(z, u)\right|^{2} w(u) w(z) d u d z \stackrel{\mathbb{P}}{\longrightarrow} 0 .
\end{aligned}
$$

for some random functions $k_{t, \kappa}(z, u)$ and $c_{t, \kappa}(z, u)$, some $t \in \mathbb{N}_{+} \cap[0, T]$ and $\kappa \in[0,1]$, and with $w$ being the weight function for the $\mathcal{L}^{2}(w)$ space defined in $(45)$.

\subsection{Localization}

Assumption SA. We have Assumption $A$ with $T_{1}=\infty$. Furthermore, the processes $\left(\alpha^{(j)}\right)_{j \geq 0}$, $\left(\beta^{(j)}\right)_{j \geq 1},\left(\gamma^{(j)}\right)_{j \geq 1}$, and $\left(\widetilde{\sigma}^{(j)}\right)_{j \geq 1}$ are uniformly bounded on $[0, T]$, and $\left|\sigma_{t}^{(0)}\right|$ is bounded from below by a positive constant on $[0, T]$.

We will prove the results under the stronger Assumption SA. A standard localization argument then can be used to show that they continue to hold under the weaker Assumption A.

\subsection{Notation and Decomposition}

We start with introducing some notation that will be used in the proofs. Throughout, we will use the shorthand notation $\mathbb{E}_{t, i}^{n}(\cdot)=\mathbb{E}\left(\cdot \mid \mathcal{F}_{t-1+(i-1) / n}\right)$ for $t \in \mathbb{N}_{+}$. For a generic process $Z_{t}$, we set

$$
Z_{t, \kappa, n}=Z_{t-1+\frac{\lfloor\kappa n\rfloor-k_{n}}{n}}, \quad t \in \mathbb{N}_{+}, \kappa \in[0,1] .
$$

and

$$
\Delta_{t, i}^{n, 2} Z=Z_{(t-1)+i / n}-Z_{(t-1)+(i-2) / n}, \quad t \in \mathbb{N}_{+}, \quad i=2, \ldots, n .
$$

We denote the spot variances of the asset prices by

$$
V_{t}^{(0)}=\left(\sigma_{t}^{(0)}\right)^{2}, \quad V_{t}^{(j)}=\left(\beta_{t}^{(j)}\right)^{2} V_{t}^{(0)}+\gamma_{t}^{(j)}\left(\gamma_{t}^{(j)}\right)^{\top}+\left(\widetilde{\sigma}_{t}^{(j)}\right)^{2}, j=1, \ldots, N,
$$

and their continuous martingale components by

$$
X_{t}^{c,(j)}=\int_{0}^{t} \beta_{s}^{(j)} \sigma_{s}^{(0)} d W_{s}^{(0)}+1_{\{j \geq 1\}} \int_{0}^{t} \gamma_{s}^{(j)} d B_{s}+1_{\{j \geq 1\}} \int_{0}^{t} \widetilde{\sigma}_{s}^{(j)} d \widetilde{W}_{s}^{(j)}, \quad k=0,1, \ldots, N,
$$

where we used the normalization $\beta_{s}^{(0)}=1$, for $s \in[0, T]$. We further set for $j=1, \ldots, N$ :

$$
C_{t}^{(j)}=\left(\beta_{t}^{(j)}-1\right) V_{t}^{(0)}, \quad t \in \mathbb{R}_{+}
$$


and for $t \in \mathbb{N}_{+}$and $\kappa \in[0,1]$ :

$$
\widetilde{C}_{t, \kappa}^{(j)}=\frac{n}{\left|\widetilde{\mathcal{I}}_{\kappa}^{n}\right|} \sum_{i \in \widetilde{\mathcal{I}}_{\kappa}^{n}} \int_{t-1+(i-1) \Delta_{n}}^{t-1+i \Delta_{n}}\left(\beta_{s}^{(j)}-1\right) V_{s}^{(0)} d s, \quad \widetilde{V}_{t, \kappa}^{(j)}=\frac{n}{\left|\mathcal{I}_{\kappa}^{n}\right|} \sum_{i \in \mathcal{I}_{\kappa}^{n}} \int_{t-1+(i-1) \Delta_{n}}^{t-1+i \Delta_{n}} V_{s}^{(j)} d s .
$$

as well as

$$
\bar{C}_{t, \kappa}^{(j)}(2)=\frac{n}{2\left|\widetilde{\mathcal{I}}_{\kappa}^{n}\right|} \sum_{i \in \widetilde{\mathcal{I}}_{\kappa}^{n}}\left[\left(\Delta_{t, i}^{n, 2} X^{c,(j)}-\Delta_{t, i}^{n, 2} X^{c,(0)}\right) \Delta_{t, i}^{n, 2} X^{c,(0)}\right], \bar{V}_{t, \kappa}^{(j)}=\frac{n}{\left|\mathcal{I}_{\kappa}^{n}\right|} \sum_{i \in \mathcal{I}_{\kappa}^{n}}\left(\Delta_{t, i}^{n} X^{c,(j)}\right)^{2} .
$$

We similarly define $\breve{\widetilde{C}}_{t, \kappa}^{(j)}$ and $\breve{\widetilde{V}}_{t, \kappa}^{(0)}$ as well as $\breve{C}_{t, \kappa}^{(j)}$ and $\breve{V}_{t, \kappa}^{(0)}$ from $\breve{C}_{t, \kappa}^{(j)}$ and $\breve{V}_{t, \kappa}^{(0)}$. Finally, we denote for $t \in \mathbb{N}_{+}$and $\kappa \in[0,1]$ :

$$
\begin{aligned}
& \bar{\eta}_{t, i}^{n,(j)}= \sigma_{t, \kappa, n}^{(0)} \gamma_{t, \kappa, n}^{(j)} n \Delta_{t, i}^{n} W^{(0)} \Delta_{t, i}^{n} B+\sigma_{t, \kappa, n}^{(0)} \widetilde{\sigma}_{t, \kappa, n}^{(j)} n \Delta_{t, i}^{n} W^{(0)} \Delta_{t, i}^{n} \widetilde{W}^{(j)} \\
&+\left(\beta_{t, \kappa, n}^{(j)}-1\right) V_{t, \kappa, n}^{(0)}\left(n\left(\Delta_{t, i}^{n} W^{(0)}\right)^{2}-1\right), k=1, \ldots, N \\
& \bar{\eta}_{t, i}^{n,(j)}(2)= \frac{1}{2} \sigma_{t, \kappa, n}^{(0)} \gamma_{t, \kappa, n}^{(j)} n \Delta_{t, i}^{n, 2} W^{(0)} \Delta_{t, i}^{n, 2} B+\frac{1}{2} \sigma_{t, \kappa, n}^{(0)} \widetilde{\sigma}_{t, \kappa, n}^{(j)} n \Delta_{t, i}^{n, 2} W^{(0)} \Delta_{t, i}^{n, 2} \widetilde{W}^{(j)} \\
&+ \frac{1}{2}\left(\beta_{t, \kappa, n}^{(j)}-1\right) V_{t, \kappa, n}^{(0)}\left(n\left(\Delta_{t, i}^{n, 2} W^{(0)}\right)^{2}-2\right), k=1, \ldots, N \\
& \bar{\eta}_{t, i}^{n,(0)}=V_{t, \kappa, n}^{(0)}\left(n\left(\Delta_{t, i}^{n} W^{(0)}\right)^{2}-1\right),
\end{aligned}
$$

and we use them to define the following processes:

$$
\begin{aligned}
\mathcal{C}_{t, \kappa}^{(j)}(2) & =\frac{1}{\left|\widetilde{\mathcal{I}}_{\kappa}^{n}\right|} \sum_{i \in \widetilde{\mathcal{I}}_{\kappa}^{n}} \bar{\eta}_{t, i}^{n,(j)}(2), \breve{\mathcal{C}}_{t, \kappa}^{(j)}=\frac{1}{\left|\mathcal{I}_{\kappa}^{n}\right|} \sum_{i \in \mathcal{I}_{\kappa}^{n}} \bar{\eta}_{t, i}^{n,(j)}\left(1_{\left\{i \in \mathcal{O}_{\kappa}^{n}\right\}}-1_{\left\{i \in \mathcal{E}_{\kappa}^{n}\right\}}\right) \\
\mathcal{V}_{t, \kappa}^{(0)} & =\frac{1}{\left|\mathcal{I}_{\kappa}^{n}\right|} \sum_{i \in \mathcal{I}_{\kappa}^{n}} \bar{\eta}_{t, i}^{n,(0)}, \breve{\mathcal{V}}_{t, \kappa}^{(0)}=\frac{1}{\left|\mathcal{I}_{\kappa}^{n}\right|} \sum_{i \in \mathcal{I}_{\kappa}^{n}} \bar{\eta}_{t, i}^{n,(0)}\left(1_{\left\{i \in \mathcal{O}_{\kappa}^{n}\right\}}-1_{\left\{i \in \mathcal{E}_{\kappa}^{n}\right\}}\right) .
\end{aligned}
$$

\subsection{Preliminary Results}

We start with establishing some preliminary results about the moments of components of the differences $\widehat{C}_{t, \kappa}^{(j)}(2)-C_{t, \kappa, n}^{(j)}$ and $\widehat{V}_{t, \kappa}^{(j)}-V_{t, \kappa, n}^{(j)}$ as well as $\breve{C}_{t, \kappa}^{(j)}$ and $\breve{V}_{t, \kappa}^{(0)}$.

Lemma 1. Assume Assumption $S A$ holds. For $j, l=0,1, \ldots, N$ and $p \geq 1$, we have:

$$
\begin{aligned}
& \mathbb{E}_{t,\lfloor\kappa n\rfloor-k_{n}+1}^{n}\left|\widehat{C}_{t, \kappa}^{(j)}(2)-\bar{C}_{t, \kappa}^{(j)}(2)\right|^{p}+\mathbb{E}_{t,\lfloor\kappa n\rfloor-k_{n}+1}^{n}\left|\widehat{V}_{t, \kappa}^{(j, l)}-\bar{V}_{t, \kappa}^{(j)}\right|^{p}+\mathbb{E}_{t,\lfloor\kappa n\rfloor-k_{n}+1}^{n}\left|\breve{C}_{t, \kappa}^{(j)}-\breve{C}_{t, \kappa}^{(j)}\right|^{p} \\
& \quad+\mathbb{E}_{t,\lfloor\kappa n\rfloor-k_{n}+1}^{n}\left|\breve{V}_{t, \kappa}^{(0, l)}-\overline{\breve{V}}_{t, \kappa}^{(0)}\right|^{p} \leq K\left(k_{n}^{1-p} \Delta_{n}^{1+p(2 \varpi-1)} \vee \Delta_{n}^{p+p(2 \varpi-1)}\right), \quad t=1, \ldots, T, \kappa \in[0,1],
\end{aligned}
$$

for some positive constant $K$ which can depend on $p$ and $\varpi$ but does not depend on $k$. 
Proof of Lemma 1. We show only the bound for the terms involving $\widehat{C}_{t, \kappa}^{(j)}(2)$ and $\breve{C}_{t, \kappa}^{(j)}$, with the result for the terms involving $\widehat{V}_{t, \kappa}^{(j, l)}$ and $\breve{V}_{t, \kappa}^{(0, l)}$ established analogously. For $j, l=0,1, \ldots, N$, we can make the decompositions:

$$
\begin{aligned}
& \Delta_{t, i}^{n} X^{(j)} \Delta_{t, i}^{n} X^{(l)} 1_{\left\{\mathcal{A}_{t, i}^{(j, l)}\right\}}-\Delta_{t, i}^{n} X^{c,(j)} \Delta_{t, i}^{n} X^{c,(l)}=-\Delta_{t, i}^{n} X^{c,(j)} \Delta_{t, i}^{n} X^{c,(l)} 1_{\left\{\left(\mathcal{A}_{t, i}^{(j, l)}\right)^{c}\right\}} \\
& \quad+\Delta_{t, i}^{n} X^{d,(j)} \Delta_{t, i}^{n} X^{d,(l)} 1_{\left\{\mathcal{A}_{t, i}^{(j, l)}\right\}}+\left(\Delta_{t, i}^{n} X^{c,(j)} \Delta_{t, i}^{n} X^{d,(l)}+\Delta_{t, i}^{n} X^{d,(j)} \Delta_{t, i}^{n} X^{c,(l)}\right) 1_{\left\{\mathcal{A}_{t, i}^{(j, l)}\right\}}, \\
& \Delta_{t, i}^{n, 2} X^{(j)} \Delta_{t, i}^{n, 2} X^{(l)} 1_{\left\{\mathcal{A}_{t, i-1}^{(j, l)} \cap \mathcal{A}_{t, i}^{(j, l)}\right\}}-\Delta_{t, i}^{n, 2} X^{c,(j)} \Delta_{t, i}^{n, 2} X^{c,(l)} \\
& =-\Delta_{t, i}^{n, 2} X^{c,(j)} \Delta_{t, i}^{n, 2} X^{c,(l)} 1_{\left\{\left(\mathcal{A}_{t, i-1}^{(j, l)}\right)^{c} \cup\left(\mathcal{A}_{t, i}^{(j, l)}\right)^{c}\right\}}+\Delta_{t, i}^{n, 2} X^{d,(j)} \Delta_{t, i}^{n, 2} X^{d,(l)} 1_{\left\{\mathcal{A}_{t, i-1}^{(j, l)} \cap \mathcal{A}_{t, i}^{(j, l)}\right\}} \\
& \quad+\left(\Delta_{t, i}^{n, 2} X^{c,(j)} \Delta_{t, i}^{n, 2} X^{d,(l)}+\Delta_{t, i}^{n, 2} X^{d,(j)} \Delta_{t, i}^{n, 2} X^{c,(l)}\right) 1_{\left\{\mathcal{A}_{t, i-1}^{(j, l)} \cap \mathcal{A}_{t, i}^{(j, l)}\right\}} .
\end{aligned}
$$

Next, using the bounds for the continuous and jump components of Itô semimartingales in Section 2.1.5 of Jacod and Protter (2012), together with Hölder's inequality, we get for $p \geq 1$ and arbitrary small $\iota>0$ :

$$
\begin{gathered}
\mathbb{E}_{t, i}^{n}\left(\left|\Delta_{t, i}^{n} X^{c,(j)} \Delta_{t, i}^{n} X^{c,(l)}\right|^{p} 1_{\left\{\left(\mathcal{A}_{t, i}^{(j, l)}\right)^{c}\right\}}\right) \leq K \Delta_{n}^{p+1-\iota} . \\
\mathbb{E}_{t, i}^{n}\left(\left|\Delta_{t, i}^{n} X^{c,(j)} \Delta_{t, i}^{n} X^{d,(l)}\right|^{p} 1_{\left\{\mathcal{A}_{t, i}^{(j, l)}\right\}}\right) \leq K \Delta_{n}^{p / 2+p \varpi+1-\iota} . \\
\mathbb{E}_{t, i}^{n}\left(\left|\Delta_{t, i}^{n} X^{d,(j)} \Delta_{t, i}^{n} X^{d,(l)}\right|^{p} 1_{\left\{\mathcal{A}_{t, i}^{(j, l)}\right\}}\right) \leq K \Delta_{n}^{1+2 p \varpi} .
\end{gathered}
$$

Combining these bounds, and using successive application of Burkholder-Gundy-Davis inequalities as well as inequality in means, the result of the lemma follows.

Lemma 2. Assume Assumption $S A$ holds. For $j=0,1, \ldots, N, t=1, \ldots, T$ and $\kappa \in[0,1]$, we have:

$$
\begin{aligned}
& \mathbb{E}_{t,\lfloor\kappa n\rfloor-k_{n}+1}^{n}\left|\bar{C}_{t, \kappa}^{(j)}(2)-\widetilde{C}_{t, \kappa}^{(j)}\right|^{p}+\mathbb{E}_{t,\lfloor\kappa n\rfloor-k_{n}+1}^{n}\left|\bar{V}_{t, \kappa}^{(j)}-\widetilde{V}_{t, \kappa}^{(j)}\right|^{p}+\mathbb{E}_{t,\lfloor\kappa n\rfloor-k_{n}+1}^{n}\left|\breve{C}_{t, \kappa}^{(j)}\right|^{p} \\
& \quad+\mathbb{E}_{t,\lfloor\kappa n\rfloor-k_{n}+1}^{n}\left|\mathcal{C}_{t, \kappa}^{(j)}(2)\right|^{p}+\mathbb{E}_{t,\lfloor\kappa n\rfloor-k_{n}+1}^{n}\left|\mathcal{V}_{t, \kappa}^{(0)}\right|^{p}+\mathbb{E}_{t,\lfloor\kappa n\rfloor-k_{n}+1}^{n}\left|\breve{\mathcal{V}}_{t, \kappa}^{(0)}\right|^{p} \\
& \mathbb{E}_{t,\lfloor\kappa n\rfloor-k_{n}+1}^{n}\left|\breve{C}_{t, \kappa}^{(j)}-\breve{\widetilde{C}}_{t, \kappa}^{(j)}\right|^{p}+\mathbb{E}_{t,\lfloor\kappa n\rfloor-k_{n}+1}^{n}\left|\breve{V}_{t, \kappa}^{(0)}-\breve{\widetilde{V}}_{t, \kappa}^{(0)}\right|^{p} \leq K k_{n}^{-p / 2}, p \geq 2,
\end{aligned}
$$

for some positive constant $K$ which can depend on $p$ but does not depend on $j$. Furthermore, we have the following decompositions

$$
\begin{gathered}
\bar{C}_{t, \kappa}^{(j)}(2)=\widetilde{C}_{t, \kappa}^{(j)}+\mathcal{C}_{t, \kappa}^{(j)}(2)+R_{t, \kappa, n}^{(j)}, \bar{V}_{t, \kappa}^{(0)}=\widetilde{V}_{t, \kappa}^{(0)}+\mathcal{V}_{t, \kappa}^{(0)}+R_{t, \kappa, n}, \\
\breve{C}_{t, \kappa}^{(j)}=\breve{\widetilde{C}}_{t, \kappa}^{(j)}+\breve{\mathcal{C}}_{t, \kappa}^{(j)}+\breve{R}_{t, \kappa, n}^{(j)}, \breve{V}_{t, \kappa}^{(0)}=\breve{\widetilde{V}}_{t, \kappa}^{(0)}+\breve{V}_{t, \kappa}^{(0)}+\breve{R}_{t, \kappa, n},
\end{gathered}
$$

where for $\mathcal{R}_{t, \kappa, n}=R_{t, \kappa, n}^{(k)}, R_{t, \kappa, n}, \breve{R}_{t, \kappa, n}^{(k)}$ or $\breve{R}_{t, \kappa, n}$, we have

$$
\mathbb{E}_{t,\lfloor\kappa n\rfloor-k_{n}+1}^{n}\left|\mathcal{R}_{t, \kappa, n}\right|^{p} \leq \frac{K}{k_{n}^{p-2} n}, p \geq 2,
$$


and for any bounded function $\omega:[0,1] \rightarrow \mathbb{R}_{+}$, we also have

$$
\mathbb{E}\left|\sum_{s=k_{n}}^{n} \omega\left(s \Delta_{n}\right) \mathcal{R}_{t, s \Delta_{n}, n}\right|^{2} \leq K k_{n}
$$

Proof of Lemma 2. The first result of the lemma follows by (successive) use of Burkholder-DavisGundy inequality. We now show the remaining claims for $\bar{C}_{t, \kappa}^{(j)}(2)$ only, with the corresponding result for $\breve{C}_{t, \kappa}^{(j)}, \bar{V}_{t, \kappa}^{(0)}$ and $\breve{V}_{t, \kappa}^{(0)}$ being established in an analogous way. Using Itô's lemma, we have

$$
n\left(\Delta_{t, i}^{n, 2} X^{c,(j)}-\Delta_{t, i}^{n, 2} X^{c,(0)}\right) \Delta_{t, i}^{n, 2} X^{c,(0)}=n \Delta_{t, i}^{n, 2} C^{(j)}+\eta_{t, i}^{n,(j)}(2),
$$

where the term $\eta_{t, i}^{n,(j)}(2)$ satisfies

$$
\mathbb{E}_{t, i-1}^{n}\left(\eta_{t, i}^{n,(j)}(2)-\bar{\eta}_{t, i}^{n,(k)}(2)\right)=0, \quad \mathbb{E}_{t, i-1}^{n}\left|\eta_{t, i}^{n,(j)}(2)-\bar{\eta}_{t, i}^{n,(j)}(2)\right|^{p} \leq K k_{n} / n, p \geq 2,
$$

and for the second result we made use of the smoothness in expectation assumption for the processes $\sigma_{t}^{(0)}, \beta_{t}^{(j)}, \gamma_{t}^{(j)}$ and $\widetilde{\sigma}_{t}^{(j)}$ as well as Burkholder-Davis-Gundy inequality. From here the result of the lemma follows.

Lemma 3. Assume Assumption $S A$ holds. For $j=0,1, \ldots, N, t=1, \ldots, T$ and $\kappa \in[0,1]$, we have

$$
\begin{aligned}
& \mathbb{E}_{t,\lfloor\kappa n\rfloor-k_{n}+1}^{n}\left(\left|\widetilde{C}_{t, \kappa}^{(j)}-C_{t, \kappa, n}^{(j)}\right|^{p} 1_{\{k \neq 0\}}\right)+\mathbb{E}_{t,\lfloor\kappa n\rfloor-k_{n}+1}^{n}\left|\widetilde{V}_{t, \kappa}^{(j)}-V_{t, \kappa, n}^{(j)}\right|^{p} \\
& \quad+\mathbb{E}_{t,\lfloor\kappa n\rfloor-k_{n}+1}^{n}\left(\left|\breve{\widetilde{C}}_{t, \kappa}^{(j)}\right|^{p} 1_{\{k \neq 0\}}\right)+\mathbb{E}_{t,\lfloor\kappa n\rfloor-k_{n}+1}^{n}\left|\breve{\widetilde{V}}_{t, \kappa}^{(j)}\right|^{p} \leq K k_{n} / n, \quad p \geq 2,
\end{aligned}
$$

for some positive constant $K$ which can depend on $p$ but does not depend on $j$. In addition, for $\omega:[0,1] \rightarrow \mathbb{R}_{+}$that is Lipschitz continuous and $t=1, \ldots, T$, we have

$$
\frac{1}{n-k_{n}+1} \sum_{s=k_{n}}^{n}\left[\omega\left(s \Delta_{n}\right)\left(\frac{\widetilde{C}_{t, s \Delta_{n}}^{(j)}}{\widetilde{V}_{t, s \Delta_{n}}^{(0)}}\right)^{2}\right]=\int_{t-1}^{t} \omega(s-t+1)\left(\beta_{s}^{(j)}-1\right)^{2} d s+O_{p}\left(\frac{k_{n}}{n}\right)
$$

with $j=1, \ldots, N$.

Proof of Lemma 3. Using Assumption SA regarding the smoothness in expectation of the processes $\beta_{t}^{(j)}$ and $\sigma_{t}^{(0)}$ as well as the boundedness of these processes, we can write

$$
\begin{gathered}
\left|\mathbb{E}_{t,\lfloor\kappa n\rfloor-k_{n}+1}^{n}\left(\widetilde{C}_{t, \kappa}^{(j)}-C_{t, \kappa, n}^{(j)}\right)\right|+\left|\mathbb{E}_{t,\lfloor\kappa n\rfloor-k_{n}+1}^{n}\left(\widetilde{V}_{t, \kappa}^{(j)}-V_{t, \kappa, n}^{(j)}\right)\right| \leq K k_{n} / n, \\
\mathbb{E}_{t,\lfloor\kappa n\rfloor-k_{n}+1}^{n}\left|\widetilde{C}_{t, \kappa}^{(j)}-C_{t, \kappa, n}^{(j)}\right|^{p}+\mathbb{E}_{t,\lfloor\kappa n\rfloor-k_{n}+1}^{n}\left|\widetilde{V}_{t, \kappa}^{(j)}-V_{t, \kappa, n}^{(j)}\right|^{p} \leq K k_{n} / n, \quad p \geq 2,
\end{gathered}
$$

where the constant $K$ does not depend on $\kappa$. From here the results of the lemma follow directly by taking into account that $\omega$ is Lipschitz continuous. 
Lemma 4. Assume Assumption $S A$ holds and $k_{n} \Delta_{n}^{2-4 \varpi} \rightarrow \infty$. For $j, l=0,1, \ldots, N$ and $p \geq 1$, we have:

$$
\mathbb{E}_{t,\lfloor\kappa n\rfloor-k_{n}+1}^{n}\left|\frac{1}{\left|\mathcal{I}_{\kappa}^{n}\right|} \sum_{i \in \mathcal{I}_{\kappa}^{n}}\left(\Delta_{t, i}^{n} X^{(0)} \Delta_{t, i}^{n} X^{(j)} 1_{\left\{\mathcal{A}_{t, i}^{(0, l)}\right\}}\right)^{2}\right|^{p} \leq K, t=1, \ldots, T, \kappa \in[0,1] .
$$

Proof of Lemma 4. The result of the lemma follows by application of the bounds in (81)(83) together with an application of Burkholder-Davis-Gundy inequalities, and further taking into account that $k_{n} \Delta_{n}^{2-4 \varpi} \rightarrow \infty$.

\subsection{Proof of Theorem 1}

Second-order Taylor expansion yields for $j=1, \ldots, N$ :

$$
\begin{aligned}
\left(\frac{\widehat{C}_{t, \kappa}^{(j)}(2)}{\widehat{V}_{t, \kappa}^{(0, j)}}\right)^{2} 1_{\left\{\widehat{V}_{t, \kappa}^{(0, j)}>\alpha_{n}\right\}}-\left(\frac{\widetilde{C}_{t, \kappa}^{(j)}}{\widetilde{V}_{t, \kappa}^{(0)}}\right)^{2}=Z_{t, \kappa, n}^{(j)}+B_{t, \kappa, n}^{(j)} & \\
& -\left(\left(\frac{\widetilde{C}_{t, \kappa}^{(j)}}{\widetilde{V}_{t, \kappa}^{(0)}}\right)^{2}+Z_{t, \kappa, n}^{(j)}+B_{t, \kappa, n}^{(j)}\right) 1_{\left\{\widehat{V}_{t, \kappa}^{(0, j)} \leq \alpha_{n}\right\}}+\bar{R}_{t, \kappa, n}^{(j)},
\end{aligned}
$$

where

$$
\begin{aligned}
Z_{t, \kappa, n}^{(j)} & =2 \frac{\widetilde{C}_{t, \kappa}^{(j)}}{\widetilde{V}_{t, \kappa}^{(0)}} \frac{1}{\widetilde{V}_{t, \kappa}^{(0)}}\left(\widehat{C}_{t, \kappa}^{(j)}(2)-\widetilde{C}_{t, \kappa}^{(j)}-\frac{\widetilde{C}_{t, \kappa}^{(j)}}{\widetilde{V}_{t, \kappa}^{(0)}}\left(\widehat{V}_{t, \kappa}^{(0, j)}-\widetilde{V}_{t, \kappa}^{(0)}\right)\right), \\
B_{t, \kappa, n}^{(j)} & =3 \frac{\left(\widetilde{C}_{t, \kappa}^{(j)}\right)^{2}}{\left(\widetilde{V}_{t, \kappa}^{(0)}\right)^{4}}\left(\widehat{V}_{t, \kappa}^{(0, j)}-\widetilde{V}_{t, \kappa}^{(0)}\right)^{2}+\frac{1}{\left(\widetilde{V}_{t, \kappa}^{(0)}\right)^{2}}\left(\widehat{C}_{t, \kappa}^{(j)}(2)-\widetilde{C}_{t, \kappa}^{(j)}\right)^{2} \\
& -4 \frac{\widetilde{C}_{t, \kappa}^{(j)}}{\left(\widetilde{V}_{t, \kappa}^{(0)}\right)^{3}}\left(\widehat{V}_{t, \kappa}^{(0, j)}-\widetilde{V}_{t, \kappa}^{(0)}\right)\left(\widehat{C}_{t, \kappa}^{(j)}(2)-\widetilde{C}_{t, \kappa}^{(j)}\right),
\end{aligned}
$$

and the residual term $\bar{R}_{t, \kappa, n}^{(j)}$ satisfies

$$
\begin{aligned}
\left|\bar{R}_{t, \kappa, n}^{(j)}\right| \leq & K \alpha_{n}^{-4}\left(\left|\widehat{V}_{t, \kappa}^{(0, j)}-\widetilde{V}_{t, \kappa}^{(0)}\right|^{3} \vee\left|\widehat{V}_{t, \kappa}^{(0, j)}-\widetilde{V}_{t, \kappa}^{(0)}\right|^{6}\right) \\
& +K \alpha_{n}^{-4}\left|\widehat{C}_{t, \kappa}^{(j)}(2)-\widetilde{C}_{t, \kappa}^{(j)}\right|\left(\left|\widehat{V}_{t, \kappa}^{(0, j)}-\widetilde{V}_{t, \kappa}^{(0)}\right|^{2} \vee\left|\widehat{V}_{t, \kappa}^{(0, j)}-\widetilde{V}_{t, \kappa}^{(0)}\right|^{4}\right) \\
& +K \alpha_{n}^{-4}\left|\widehat{C}_{t, \kappa}^{(j)}(2)-\widetilde{C}_{t, \kappa}^{(j)}\right|^{2}\left(\left|\widehat{V}_{t, \kappa}^{(0, j)}-\widetilde{V}_{t, \kappa}^{(0)}\right| \vee\left|\widehat{V}_{t, \kappa}^{(0, j)}-\widetilde{V}_{t, \kappa}^{(0)}\right|^{4}\right),
\end{aligned}
$$

where the constant $K$ does not depend on $j$. Finally, in what follows we use the following notation

$$
\begin{aligned}
\bar{Z}_{t, \kappa, n}^{(j)}= & 2\left(\beta_{t, \kappa, n}^{(j)}-1\right)^{2} \frac{n}{\left|\widetilde{\mathcal{I}}_{\kappa}^{n}\right|} \sum_{i \in \widetilde{\mathcal{I}}_{\kappa}^{n}}\left[\Delta_{t, i-1}^{n} W^{(0)} \Delta_{t, i}^{n} W^{(0)}\right] \\
& +\left(\beta_{t, \kappa, n}^{(j)}-1\right) \frac{n}{\left|\widetilde{\mathcal{I}}_{\kappa}^{n}\right|} \sum_{i \in \widetilde{\mathcal{I}}_{\kappa}^{n}}\left[\frac{\gamma_{t, \kappa, n}^{(j)}}{\sigma_{t, \kappa, n}^{(0)}} \Delta_{t, i}^{n, 2} W^{(0)} \Delta_{t, i}^{n, 2} B+\frac{\widetilde{\sigma}_{t, \kappa, n}^{(j)}}{\sigma_{t, \kappa, n}^{(0)}} \Delta_{t, i}^{n, 2} W^{(0)} \Delta_{t, i}^{n, 2} \widetilde{W}^{(j)}\right]
\end{aligned}
$$


The proof of the theorem consists of a sequence of lemmas.

Lemma 5. Under Assumption $S A$, and provided $k_{n} \Delta_{n} \rightarrow 0$ and $k_{n} \Delta_{n}^{1-2 \varpi} \rightarrow \infty$, we have for $t=1, \ldots, T$ and $\kappa \in[0,1]:$

$$
\frac{1}{N} \sum_{j=1}^{N} \bar{R}_{t, \kappa, n}^{(j)}+\frac{1}{n-k_{n}+1} \sum_{s=k_{n}}^{n} \frac{1}{N} \sum_{j=1}^{N}\left|\bar{R}_{t, s \Delta_{n}, n}^{(j)}\right|=O_{p}\left(\frac{1}{\alpha_{n}^{4}}\left(k_{n}^{-2} \Delta_{n}^{1+3(2 \varpi-1)} \bigvee \frac{1}{k_{n}^{3 / 2}}\right)\right)
$$

Proof of Lemma 5. The result of the lemma follows by an application of Hölder's inequality and making use of the bounds of Lemmas 1-2 as well as the inequality in (99).

Lemma 6. Under Assumption $S A$, and provided $\varpi \in(1 / 4,1 / 2), \varrho \in(0,1 / 2)$ and $k_{n} \Delta_{n}^{2-4 \varpi} \rightarrow \infty$, we have for $t=1, \ldots, T, \kappa \in[0,1]$ and some arbitrary small $\iota>0$ :

$$
\widehat{B}_{t, \kappa}^{N}-\frac{1}{N} \sum_{j=1}^{N} B_{t, \kappa, n}^{(j)}=O_{p}\left(\frac{1}{\alpha_{n}^{4}}\left(\frac{\Delta_{n}^{1+2(2 \varpi-1)-\iota}}{k_{n}} \bigvee\left(\frac{k_{n}}{n}\right)^{1-\iota} \bigvee \frac{1}{k_{n}^{3 / 2}}\right)\right),
$$

and further for any bounded function $\omega:[0,1] \rightarrow \mathbb{R}:$

$$
\begin{array}{r}
\frac{1}{n-k_{n}+1} \sum_{s=k_{n}}^{n}\left[\omega\left(s \Delta_{n}\right)\left(\widehat{B}_{t, s \Delta_{n}}^{N}-\frac{1}{N} \sum_{j=1}^{N} B_{t, s \Delta_{n}, n}^{(j)}\right)\right] \\
=O_{p}\left(\frac{1}{\alpha_{n}^{4}}\left(\frac{\Delta_{n}^{1+2(2 \varpi-1)-\iota}}{k_{n}} \bigvee\left(\frac{k_{n}}{n}\right)^{1-\iota} \bigvee \frac{1}{k_{n}^{3 / 2}}\right)\right) .
\end{array}
$$

Proof of Lemma 6. We start with defining the processes for $j=1, \ldots, N$ (recall the notation in equations (73)-(77)):

$$
\begin{gathered}
\mathcal{B}_{t, \kappa, n}^{1,(j)}=-\frac{\left(\beta_{t, \kappa, n}^{(j)}-1\right)^{2}}{\left(V_{t, \kappa, n}^{(0)}\right)^{2}}\left(\breve{\mathcal{V}}_{t, \kappa}^{(0)}\right)^{2}+\frac{3}{2\left(V_{t, \kappa, n}^{(0)}\right)^{2}}\left(\breve{\mathcal{C}}_{t, \kappa}^{(j)}\right)^{2} \\
\mathcal{B}_{t, \kappa, n}^{2,(j)}=3 \frac{\left(\beta_{t, \kappa, n}^{(j)}-1\right)^{2}}{\left(V_{t, \kappa, n}^{(0)}\right)^{2}}\left(\mathcal{V}_{t, \kappa}^{(0)}\right)^{2}+\frac{1}{\left(V_{t, \kappa, n}^{(0)}\right)^{2}}\left(\mathcal{C}_{t, \kappa}^{(j)}(2)\right)^{2}-4 \frac{\left(\beta_{t, \kappa, n}^{(j)}-1\right)}{\left(V_{t, \kappa, n}^{(0)}\right)^{2}} \mathcal{V}_{t, \kappa}^{(0)} \mathcal{C}_{t, \kappa}^{(j)}(2)
\end{gathered}
$$

By direct calculation:

$$
\begin{aligned}
& \mathbb{E}_{t,\lfloor\kappa n\rfloor-k_{n}+1}^{n}\left(\breve{\mathcal{C}}_{t, \kappa}^{(j)}\right)^{2}= \frac{1}{\left|\mathcal{I}_{\kappa}^{n}\right|} V_{t, \kappa, n}^{(0)} \gamma_{t, \kappa, n}^{(j)}\left(\gamma_{t, \kappa, n}^{(j)}\right)^{\top}+\frac{1}{\left|\mathcal{I}_{\kappa}^{n}\right|} V_{t, \kappa, n}^{(0)}\left(\widetilde{\sigma}_{t, \kappa, n}^{(j)}\right)^{2} \\
&+\frac{1}{\left|\mathcal{I}_{\kappa}^{n}\right|}\left(V_{t, \kappa, n}^{(0)}\right)^{2}\left(\beta_{t, \kappa, n}^{(j)}-1\right)^{2} \\
& \mathbb{E}_{t,\lfloor\kappa n\rfloor-k_{n}+1}^{n}\left(\mathcal{C}_{t, \kappa}^{(j)}(2)\right)^{2}=\frac{3}{2} \frac{\left|\mathcal{I}_{\kappa}^{n}\right|}{\left|\widetilde{\mathcal{I}}_{\kappa}^{n}\right|} \mathbb{E}_{t,\lfloor\kappa n\rfloor-k_{n}+1}^{n}\left(\breve{\mathcal{C}}_{t, \kappa}^{(j)}\right)^{2}, \\
& \mathbb{E}_{t,\lfloor\kappa n\rfloor-k_{n}+1}^{n}\left(\mathcal{V}_{t, \kappa}^{(0)}\right)^{2}=\mathbb{E}_{t,\lfloor\kappa n\rfloor-k_{n}+1}^{n}\left(\breve{\mathcal{V}}_{t, \kappa}^{(0)}\right)^{2}=\frac{2}{\left|\mathcal{I}_{\kappa}^{n}\right|}\left(V_{t, \kappa, n}^{(0)}\right)^{2}
\end{aligned}
$$




$$
\mathbb{E}_{t,\lfloor\kappa n\rfloor-k_{n}+1}^{n}\left(\mathcal{V}_{t, \kappa}^{(0)} \mathcal{C}_{t, \kappa}^{(j)}(2)\right)=\left(\beta_{t, \kappa, n}^{(j)}-1\right) \mathbb{E}_{t,\lfloor\kappa n\rfloor-k_{n}+1}^{n}\left(\mathcal{V}_{t, \kappa}^{(0)}\right)^{2} .
$$

From here, using the first bound in Lemma 2, we have

$$
\left|\mathbb{E}_{t,\lfloor\kappa n\rfloor-k_{n}+1}^{n}\left(\mathcal{B}_{t, \kappa, n}^{1,(j)}-\mathcal{B}_{t, \kappa, n}^{2,(j)}\right)\right| \leq \frac{K}{k_{n}^{2}}, \mathbb{E}\left[\left(\mathcal{B}_{t, \kappa, n}^{1,(j)}\right)^{2}+\left(\mathcal{B}_{t, \kappa, n}^{2,(j)}\right)^{2}\right] \leq \frac{K}{k_{n}^{2}}
$$

where the constant $K$ does not depend on $t, \kappa$ and $j$. Next, using Lemmas 1-2, Cauchy-Schwarz and Burkholder-Davis-Gundy inequalities and taking into account that $\varpi>1 / 4$ and $\varrho \in(0,1 / 2)$, we have

$$
\mathbb{E}\left|B_{t, \kappa, n}^{(j)}-\mathcal{B}_{t, \kappa, n}^{2,(j)}\right| \leq K\left(\frac{\Delta_{n}^{1+2(2 \varpi-1)}}{k_{n}} \bigvee \frac{1}{\sqrt{k_{n} n}}\right) .
$$

We proceed with analyzing the difference $\widehat{B}_{t, \kappa, n}^{N}-\frac{1}{N} \sum_{j=1}^{N} \mathcal{B}_{t, \kappa, n}^{1,(j)}$. First, using Lemma 1-4, Hölder's inequality as well as the restriction $k_{n} \Delta_{n}^{2-4 \varpi} \rightarrow \infty$ of the lemma, we have

$$
\mathbb{E}\left|\widehat{V}_{\beta, t, \kappa}^{(j)}\left(\breve{V}_{t, \kappa}^{(0, j)}\right)^{2} 1_{\left\{\breve{V}_{t, \kappa}^{(0, j)}>\alpha_{n}\right\}}\right| \leq \frac{K}{\alpha_{n}^{2}} \frac{1}{k_{n}}\left[\frac{1}{k_{n}} \bigvee \Delta_{n}^{4 \varpi-\iota}\right]
$$

for some arbitrary small $\iota>0$. We continue with introducing the following notation

$$
\begin{gathered}
\xi_{t, \kappa}^{(j, 1)}=\frac{\widehat{\beta}_{t, \kappa}^{(j)}-1}{\widehat{V}_{t, \kappa}^{(0, j)}} \breve{V}_{t, \kappa}^{(0)}-\frac{\beta_{t, \kappa, n}^{(j)}-1}{V_{t, \kappa, n}^{(0)}} \breve{\mathcal{V}}_{t, \kappa}^{(0)} \\
\xi_{t, \kappa}^{(j, 2)}=\frac{1}{\widehat{V}_{t, \kappa}^{(0, j)}} \breve{C}_{t, \kappa}^{(j)}-\frac{1}{V_{t, \kappa, n}^{(0)}} \breve{\mathcal{C}}_{t, \kappa}^{(j)}
\end{gathered}
$$

Then, we have

$$
\begin{gathered}
\left|\xi_{t, \kappa}^{(j, 1)}\right| 1_{\left\{\widehat{V}_{t, \kappa}^{(0, j)}>\alpha_{n}\right\}} \leq \frac{K}{\alpha_{n}^{2}}\left(\left|\widehat{C}_{t, \kappa}^{(j, 0)}-C_{t, \kappa, n}^{(j, 0)}\right| \vee\left|\widehat{V}_{t, \kappa}^{(0, j)}-V_{t, \kappa, n}^{(0)}\right| \vee 1\right)\left|\breve{V}_{t, \kappa}^{(0)}-\breve{\mathcal{V}}_{t, \kappa}^{(0)}\right| \\
+\frac{K}{\alpha_{n}^{2}}\left|\breve{\mathcal{V}}_{t, \kappa}^{(0)}\right|\left(\left|\widehat{C}_{t, \kappa}^{(j, 0)}-C_{t, \kappa, n}^{(j, 0)}\right| \vee\left|\widehat{V}_{t, \kappa}^{(0, j)}-V_{t, \kappa, n}^{(0)}\right|^{2} \vee\left|\widehat{V}_{t, \kappa}^{(0, j)}-V_{t, \kappa, n}^{(0)}\right|\right), \\
\left|\xi_{t, \kappa}^{(j, 2)}\right| 1_{\left\{\widehat{V}_{t, \kappa}^{(0, j)}>\alpha_{n}\right\}} \leq \frac{K}{\alpha_{n}}\left|\breve{C}_{t, \kappa}^{(j)}-\breve{\mathcal{C}}_{t, \kappa}^{(j)}\right|+\frac{K}{\alpha_{n}}\left|\breve{\mathcal{C}}_{t, \kappa}^{(j)}\right|\left|\widehat{V}_{t, \kappa}^{(0, j)}-V_{t, \kappa, n}^{(0)}\right|,
\end{gathered}
$$

where we denote

$$
\widehat{C}_{t, \kappa}^{(j, 0)}=\frac{n}{\left|\mathcal{I}_{\kappa}^{n}\right|} \sum_{i \in \mathcal{I}_{\kappa}^{n}} \Delta_{t, i}^{n} X^{(j)} \Delta_{t, i}^{n} X^{(0)} 1_{\left\{\mathcal{A}_{t, i}^{(j, 0)}\right\}}, \quad C_{t}^{(j, 0)}=\int_{0}^{t} \beta_{s}^{(j)} V_{s}^{(0)} d s, j=1, \ldots, N .
$$

From here, using Lemmas 1-3, we have

$$
\mathbb{E}_{t,\lfloor\kappa n\rfloor-k_{n}+1}^{n}\left[\left(\left|\xi_{t, \kappa}^{(j, 1)}\right|^{2}+\left|\xi_{t, \kappa}^{(j, 2)}\right|^{2}\right) 1_{\left\{\widehat{V}_{t, \kappa}^{(0, j)}>\alpha_{n}\right\}}\right] \leq \frac{K}{\alpha_{n}^{4}}\left(\frac{\Delta_{n}^{1+2(2 \varpi-1)-\iota}}{k_{n}} \bigvee \frac{1}{k_{n}^{2}} \bigvee\left(\frac{k_{n}}{n}\right)^{1-\iota}\right),
$$


for some arbitrary small $\iota>0$. Similarly, we have

$$
\mathbb{E}_{t,\lfloor\kappa n\rfloor-k_{n}+1}^{n}\left[\left(\left|\xi_{t, \kappa}^{(j, 1)}\right|\left|\breve{\mathcal{V}}_{t, \kappa}^{(0)}\right|+\left|\xi_{t, \kappa}^{(j, 2)}\right|\left|\breve{\mathcal{C}}_{t, \kappa}^{(j)}\right|\right) 1_{\left\{\widehat{V}_{t, \kappa}^{(0, j)}>\alpha_{n}\right\}}\right] \leq \frac{K}{\alpha_{n}^{2}}\left(\frac{1}{k_{n}^{3 / 2}} \vee\left(\frac{k_{n}}{n}\right)^{1-\iota} \frac{\Delta_{n}^{2 \varpi-1}}{k_{n}^{2}}\right)
$$

Furthermore, given the lower bound restriction on $V_{t}^{(0)}$ in Assumption SA, we have

$$
1_{\left\{\widehat{V}_{t, \kappa}^{(0, j)} \leq \alpha_{n}\right\}} \leq K_{p}\left|\widehat{V}_{t, \kappa}^{(0)}-\widetilde{V}_{t, \kappa}^{(0)}\right|^{p}, \quad \forall p \geq 1
$$

where the constant $K_{p}$ depends on $p$. Therefore, by making use again of Lemmas 1-3 and taking into account the restriction on $\varpi$ and $\varrho$ of the lemma, we have altogether:

$$
\mathbb{E}\left|\widehat{B}_{t, \kappa, n}^{N}-\frac{1}{N} \sum_{j=1}^{N} \mathcal{B}_{t, \kappa, n}^{1,(j)}\right| \leq \frac{K}{\alpha_{n}^{4}}\left(\frac{\Delta_{n}^{1+2(2 \varpi-1)-\iota}}{k_{n}} \bigvee \frac{1}{k_{n}^{3 / 2}} \bigvee\left(\frac{k_{n}}{n}\right)^{1-\iota}\right),
$$

where again the constant $K$ does not depend on $t, \kappa$ and $j$. Combining the above bounds, we get the first bound of the lemma in (102). For the second bound in (103) we make in addition use of the following

$$
\frac{1}{n^{2}} \sum_{s=k_{n}}^{n}\left[\omega^{2}\left(s \Delta_{n}\right)\left(\mathcal{B}_{t, s \Delta_{n}, n}^{1,(j)}-\mathcal{B}_{t, s \Delta_{n}, n}^{2,(j)}-\mathbb{E}_{t, s-k_{n}+1}^{n}\left(\mathcal{B}_{t, s \Delta_{n}, n}^{1,(j)}-\mathcal{B}_{t, s \Delta_{n}, n}^{2,(j)}\right)\right)^{2}\right] \leq \frac{K}{k_{n} n},
$$

which in turn follows by application of Cauchy-Schwarz inequality and the second bound in (110).

Lemma 7. Under Assumption $S A$, and provided $\varrho \in(0,1 / 2)$ we have for $t=1, \ldots, T$ and $\kappa \in[0,1]$ as well as some arbitrary small $\iota>0$ :

$$
\begin{aligned}
\left|\mathbb{E}_{t,\lfloor\kappa n\rfloor-k_{n}+1}^{n}\left(Z_{t, \kappa, n}^{(j)}-\bar{Z}_{t, \kappa, n}^{(j)}\right)\right| \leq K\left(\Delta_{n}^{2 \varpi} \bigvee \frac{k_{n}}{n} \bigvee\left(\frac{k_{n}}{n}\right)^{1-\iota} \frac{1}{\sqrt{k_{n}}}\right) \\
\mathbb{E}_{t,\lfloor\kappa n\rfloor-k_{n}+1}^{n}\left(Z_{t, \kappa, n}^{(j)}-\bar{Z}_{t, \kappa, n}^{(j)}\right)^{2} \leq K\left(\Delta_{n}^{2 \varpi} \bigvee\left(\frac{k_{n}}{n}\right)^{1-\iota} \frac{1}{k_{n}} \bigvee \frac{1}{k_{n}^{2}}\right)
\end{aligned}
$$

where the constant $K$ does not depend on $t, \kappa$ and $j$, and $Z_{t, \kappa, n}^{(j)}$ and $\bar{Z}_{t, \kappa, n}^{(j)}$ are defined in (97) and (100), respectively.

Proof of Lemma 7. We denote with $\mathcal{Z}_{t, \kappa, n}^{(j)}$, the counterpart of $Z_{t, \kappa, n}^{(j)}$ in which $\widehat{C}_{t, \kappa}^{(j)}(2)$ and $\widehat{V}_{t, \kappa}^{(0, j)}$ are replaced with $\bar{C}_{t, \kappa}^{(j)}(2)$ and $\bar{V}_{t, \kappa}^{(0)}$, respectively. Then, using Lemma 1 and taking into account that $\varrho \in(0,1 / 2)$, we have

$$
\mathbb{E}_{t,\lfloor\kappa n\rfloor-k_{n}+1}^{n}\left|Z_{t, \kappa, n}^{(j)}-\mathcal{Z}_{t, \kappa, n}^{(j)}\right|+\mathbb{E}_{t,\lfloor\kappa n\rfloor-k_{n}+1}^{n}\left|Z_{t, \kappa, n}^{(j)}-\mathcal{Z}_{t, \kappa, n}^{(j)}\right|^{2} \leq K \Delta_{n}^{2 \varpi},
$$


where the constant $K$ does not depend on $t, \kappa$ and $j$. Using successive conditioning and Assumption SA, we have

$$
\left|\mathbb{E}_{t,\lfloor\kappa n\rfloor-k_{n}+1}^{n}\left(\chi_{1, n} \chi_{2, n}\right)\right| \leq K \frac{k_{n}}{n}
$$

for

$$
\chi_{1, n}=\widetilde{C}_{t, \kappa}^{(j)}-C_{t, \kappa, n}^{(j)} \text { or } \widetilde{V}_{t, \kappa}^{(0)}-V_{t, \kappa, n}^{(0)}, \chi_{2, n}=\bar{C}_{t, \kappa, n}^{(j)}(2)-\widetilde{C}_{t, \kappa, n}^{(j)} \text { or } \bar{V}_{t, \kappa}^{(0)}-\widetilde{V}_{t, \kappa, n}^{(0)}
$$

From here, using Taylor expansion and Lemmas 2-3, we have

$$
\left|\mathbb{E}_{t,\lfloor\kappa n\rfloor-k_{n}+1}^{n}\left(\mathcal{Z}_{t, \kappa, n}^{(j)}-\bar{Z}_{t, \kappa, n}^{(j)}\right)\right| \leq K\left(\frac{k_{n}}{n} \bigvee\left(\frac{k_{n}}{n}\right)^{1-\iota} \frac{1}{\sqrt{k_{n}}}\right) .
$$

Similar analysis leads to

$$
\mathbb{E}_{t,\lfloor\kappa n\rfloor-k_{n}+1}^{n}\left(\mathcal{Z}_{t, \kappa, n}^{(j)}-\bar{Z}_{t, \kappa, n}^{(j)}\right)^{2} \leq K\left(\left(\frac{k_{n}}{n}\right)^{1-\iota} \frac{1}{k_{n}} \bigvee \frac{1}{k_{n}^{2}}\right) .
$$

From here the results of the lemma follow.

Lemma 8. Under Assumption $S A$, and provided $k_{n} \Delta_{n} \rightarrow 0$ and $k_{n} \Delta_{n}^{1-2 \varpi} \rightarrow \infty$, we have for $t=1, \ldots, T, \kappa \in[0,1]$ and $j=1, \ldots, N$ :

$$
\mathbb{E}_{t,\lfloor\kappa n\rfloor-k_{n}+1}^{n}\left[\left(1+\left|Z_{t, \kappa, n}^{(j)}\right|+\left|B_{t, \kappa, n}^{(j)}\right|\right) 1_{\left\{\widehat{V}_{t, \kappa}^{(0, j)} \leq \alpha_{n}\right\}}\right] \leq K\left(\frac{k_{n}}{n} \bigvee \frac{1}{k_{n}^{p / 2}} \bigvee k_{n}^{1-p-\iota} \Delta_{n}^{1+p(2 \varpi-1)-\iota}\right),
$$

for some arbitrary big $p>2$ and some arbitrary small $\iota>0$, and where the constant $K$ depends on $p$ but not on $j$, $t$ and $\kappa$.

Proof of Lemma 8. Using the inequality $1_{\left\{\widehat{V}_{t, \kappa}^{(0, j)} \leq \alpha_{n}\right\}} \leq K_{p}\left|\widehat{V}_{t, \kappa}^{(0, j)}-\widetilde{V}_{t, \kappa}^{(0)}\right|^{p}$, for arbitrary $p \geq 1$, we have

$$
\begin{aligned}
& \left(1+\left|Z_{t, \kappa, n}^{(j)}\right|+\left|B_{t, \kappa, n}^{(j)}\right|\right) 1_{\left\{\widehat{V}_{t, \kappa}^{(0, j)} \leq \alpha_{n}\right\}} \\
& \quad \leq K\left|\widehat{V}_{t, \kappa}^{(0, j)}-\widetilde{V}_{t, \kappa}^{(0)}\right|^{p}\left(1+\left|\widehat{C}_{t, \kappa}^{(j)}(2)-\widetilde{C}_{t, \kappa}^{(j)}\right|+\left|\widehat{C}_{t, \kappa}^{(j)}(2)-\widetilde{C}_{t, \kappa}^{(j)}\right|^{2}\right),
\end{aligned}
$$

for some arbitrary $p>2$ and with the constant $K$ not depending on $j, t$ and $\kappa$. From here, the result of the lemma follows by application of Lemmas 1-3 as well as the assumed relative growth conditions for $k_{n}$.

For the statement of the next lemma, we need some additional notation. We denote with $\widetilde{\operatorname{Avar}}\left(\widehat{D}_{t, \kappa}^{N}\right)$ the counterpart of $\widehat{\operatorname{Avar}}\left(\widehat{D}_{t, \kappa}^{N}\right)$ in which we replace $\widehat{\beta}_{t, \kappa}^{(j)}$ with $\beta_{t-1+\kappa}^{(j)}, \widehat{V}_{t, \kappa}^{(0)}$ with $V_{t-1+\kappa}^{(0)}$, 
$\breve{C}_{t, \kappa}^{(j)}$ with $\breve{\mathcal{C}}_{t, \kappa}^{(j)}$, and $\breve{V}_{t, \kappa}^{(0)}$ with $\breve{\mathcal{V}}_{t, \kappa}^{(0)}$. In addition, we set

$$
\begin{aligned}
\operatorname{Avar}\left(\widehat{D}_{t, \kappa}^{N}\right)= & \frac{6}{N^{2}\left|\widetilde{\mathcal{I}}_{\kappa}^{n}\right|} \sum_{j=1}^{N}\left[\left(\beta_{t-1+\kappa}^{(j)}-1\right)^{2} \frac{\left(\widetilde{\sigma}_{t-1+\kappa}^{(j)}\right)^{2}}{\left(\sigma_{t-1+\kappa}^{(0)}\right)^{2}}\right]+\frac{4}{\left|\widetilde{\mathcal{I}}_{\kappa}^{n}\right|}\left(\frac{1}{N} \sum_{j=1}^{N}\left(\beta_{t-1+\kappa}^{(j)}-1\right)^{2}\right)^{2} \\
& +\frac{6}{\left|\widetilde{\mathcal{I}}_{\kappa}^{n}\right|}\left(\frac{1}{N} \sum_{j=1}^{N} \frac{\left(\beta_{t-1+\kappa}^{(j)}-1\right) \gamma_{t-1+\kappa}^{(j)}}{\sigma_{t-1+\kappa}^{(0)}}\right)\left(\frac{1}{N} \sum_{j=1}^{N} \frac{\left(\beta_{t-1+\kappa}^{(j)}-1\right)\left(\gamma_{t-1+\kappa}^{(j)}\right)^{\top}}{\sigma_{t-1+\kappa}^{(0)}}\right)
\end{aligned}
$$

Lemma 9. Under Assumption $S A$, and provided $\varpi \in(0,1 / 2), \varrho \in(0,1 / 2)$ and $k_{n} \Delta_{n}^{2-4 \varpi} \rightarrow \infty$, we have for $t=1, \ldots, T$ and $\kappa \in[0,1]$ and some arbitrary small $\iota>0$ :

$$
\begin{gathered}
\mathbb{E}_{t,\lfloor\kappa n\rfloor-k_{n}+1}^{n}\left|\widehat{A v a r}\left(\widehat{D}_{t, \kappa}^{N}\right)-\operatorname{Avar}\left(\widehat{D}_{t, \kappa}^{N}\right)\right| \\
\leq \frac{K}{\alpha_{n}^{6}}\left(\frac{1}{\sqrt{n}} \bigvee \frac{1}{k_{n}^{3 / 2}} \bigvee \frac{\Delta_{n}^{2 \varpi-\iota}}{k_{n}} \bigvee \frac{\Delta_{n}^{1+2(2 \varpi-1)-\iota}}{k_{n}}\right), \\
\mathbb{E}_{t,\lfloor\kappa n\rfloor-k_{n}+1}^{n}\left(\widetilde{\operatorname{Avar}}\left(\widehat{D}_{t, \kappa}^{N}\right)-\operatorname{Avar}\left(\widehat{D}_{t, \kappa}^{N}\right)\right)=0, \\
\mathbb{E}_{t,\lfloor\kappa n\rfloor-k_{n}+1}^{n}\left(\left(\widetilde{\operatorname{Avar}}\left(\widehat{D}_{t, \kappa}^{N}\right)\right)-\operatorname{Avar}\left(\widehat{D}_{t, \kappa}^{N}\right)\right)^{2} \leq \frac{K}{k_{n}^{3}}
\end{gathered}
$$

Proof of Lemma 9. We start with the first bound. If we denote

$$
\begin{gathered}
\xi_{t, \kappa}^{(j, 3)}=\frac{\widehat{\beta}_{t, \kappa}^{(j)}-1}{\widehat{V}_{t, \kappa}^{(0, j)}} \breve{C}_{t, \kappa}^{(j)}-\frac{\beta_{t, \kappa, n}^{(j)}-1}{V_{t, \kappa, n}^{(0)}} \breve{\mathcal{C}}_{t, \kappa}^{(j)}, \\
\xi_{t, \kappa}^{(j, 4)}=\frac{\left(\widehat{\beta}_{t, \kappa}^{(j)}-1\right)^{2}}{\widehat{V}_{t, \kappa}^{(0, j)}} \breve{V}_{t, \kappa}^{(0)}-\frac{\left(\beta_{t, \kappa, n}^{(j)}-1\right)^{2}}{V_{t, \kappa, n}^{(0)}} \breve{\mathcal{V}}_{t, \kappa}^{(0)},
\end{gathered}
$$

then direct calculation shows

$$
\begin{gathered}
\left|\xi_{t, \kappa}^{(j, 3)}\right| 1_{\left\{\widehat{V}_{t, \kappa}^{(0, j)}>\alpha_{n}\right\}} \leq \frac{K}{\alpha_{n}^{2}}\left(\left|\widehat{C}_{t, \kappa}^{(j, 0)}-C_{t, \kappa, n}^{(j, 0)}\right| \vee\left|\widehat{V}_{t, \kappa}^{(0, j)}-V_{t, \kappa, n}^{(0)}\right| \vee 1\right)\left|\breve{C}_{t, \kappa}^{(j)}-\breve{\mathcal{C}}_{t, \kappa}^{(j)}\right| \\
+\frac{K}{\alpha_{n}^{2}}\left|\breve{\mathcal{C}}_{t, \kappa}^{(j)}\right|\left(\left|\widehat{C}_{t, \kappa}^{(j, 0)}-C_{t, \kappa, n}^{(j, 0)}\right| \vee\left|\widehat{V}_{t, \kappa}^{(0, j)}-V_{t, \kappa, n}^{(0)}\right|^{2} \vee\left|\widehat{V}_{t, \kappa}^{(0, j)}-V_{t, \kappa, n}^{(0)}\right|\right), \\
\left|\xi_{t, \kappa}^{(j, 4)}\right| 1_{\left\{\widehat{V}_{t, \kappa}^{(0, j)}>\alpha_{n}\right\}} \leq \frac{K}{\alpha_{n}^{3}}\left(\left|\widehat{C}_{t, \kappa}^{(j, 0)}-C_{t, \kappa, n}^{(j, 0)}\right|^{2} \vee\left|\widehat{V}_{t, \kappa}^{(0, j)}-V_{t, \kappa, n}^{(0)}\right|^{2} \vee 1\right)\left|\breve{V}_{t, \kappa}^{(0)}-\breve{\mathcal{V}}_{t, \kappa}^{(0)}\right| \\
+\frac{K}{\alpha_{n}^{3}}\left|\breve{V}_{t, \kappa}^{(0)}\right|\left(\left|\widehat{V}_{t, \kappa}^{(0, j)}-V_{t, \kappa, n}^{(0)}\right|^{3} \vee\left|\widehat{V}_{t, \kappa}^{(0, j)}-V_{t, \kappa, n}^{(0)}\right| \vee\left|\widehat{C}_{t, \kappa}^{(j, 0)}-C_{t, \kappa, n}^{(j, 0)}\right|^{2} \vee\left|\widehat{C}_{t, \kappa}^{(j, 0)}-C_{t, \kappa, n}^{(j, 0)}\right|\right),
\end{gathered}
$$

where we use the notation $\widehat{C}_{t, \kappa}^{(j, 0)}$ and $C_{t}^{(j, 0)}$ as defined in the proof of Lemma 6 (see equation (117)). From here, using Lemmas 1-3, we have

$$
\mathbb{E}_{t,\lfloor\kappa n\rfloor-k_{n}+1}^{n}\left[\left(\left|\xi_{t, \kappa}^{(j, 3)}\right|^{2}+\left|\xi_{t, \kappa}^{(j, 4)}\right|^{2}\right) 1_{\left\{\widehat{V}_{t, \kappa}^{(0, j)}>\alpha_{n}\right\}}\right] \leq \frac{K}{\alpha_{n}^{6}}\left(\frac{\Delta_{n}^{1+2(2 \varpi-1)-\iota}}{k_{n}} \bigvee \frac{1}{k_{n}^{2}} \bigvee\left(\frac{k_{n}}{n}\right)^{1-\iota}\right),
$$


for some arbitrary small $\iota>0$. Similarly, we have

$$
\mathbb{E}_{t,\lfloor\kappa n\rfloor-k_{n}+1}^{n}\left[\left(\left|\xi_{t, \kappa}^{(j, 3)}\right|+\left|\xi_{t, \kappa}^{(j, 4)}\right|\right)\left(\left|\breve{\mathcal{C}}_{t, \kappa}^{(j)}\right|+\left|\breve{\mathcal{V}}_{t, \kappa}^{(0)}\right|\right) 1_{\left\{\widehat{V}_{t, \kappa}^{(0)}>\alpha_{n}\right\}}\right] \leq \frac{K}{\alpha_{n}^{3}}\left(\frac{1}{k_{n}^{3 / 2}} \vee\left(\frac{k_{n}}{n}\right)^{1-\iota} \frac{\Delta_{n}^{2 \varpi-1}}{k_{n}^{2}}\right)
$$

Finally, using similar proof as that of the bound in (112), we can show

$$
\mathbb{E}\left|\left(\widehat{V}_{\beta, t, \kappa}^{(j)}\right)^{2}\left(\breve{V}_{t, \kappa}^{(0, j)}\right)^{2} 1_{\left\{\breve{V}_{t, \kappa}^{(0, j)}>\alpha_{n}\right\}}\right| \leq \frac{K}{\alpha_{n}^{4}} \frac{1}{k_{n}^{2}}\left[\frac{1}{k_{n}} \bigvee \Delta_{n}^{4 \varpi-\iota}\right]
$$

for arbitrary small $\iota>0$. From here, using again Lemmas 1-3, we get the first bound of the lemma. The second and third results of the lemma follow by direct calculation.

For the statement of the next lemma, we introduce some additional notation. We denote

$$
\widehat{Z}_{t, \kappa, n}^{(a)}=\sum_{i \in \widetilde{\mathcal{I}}_{\kappa}^{n}} \chi_{i, t, \kappa, n}^{(a)}, \widehat{Z}_{t, \kappa, n}^{(b)}=\sum_{i \in \widetilde{\mathcal{I}}_{\kappa}^{n}} \chi_{i, t, \kappa, n}^{(b)}, \widehat{Z}_{t, \kappa, n}^{(c)}=\sum_{i \in \widetilde{\mathcal{I}}_{\kappa}^{n}} \chi_{i, t, \kappa, n}^{(c)}, t=1, \ldots, T, \kappa \in[0,1],
$$

where

$$
\begin{gathered}
\chi_{i, t, \kappa, n}^{(a)}=\frac{1}{\sqrt{N}} \frac{n}{\sqrt{\left|\widetilde{\mathcal{I}}_{\kappa}^{n}\right|}} \sum_{j=1}^{N}\left[\left(\beta_{t, \kappa, n}^{(j)}-1\right) \frac{\widetilde{\sigma}_{t, \kappa, n}^{(j)}}{\sigma_{t, \kappa, n}^{(0)}} \Delta_{t, i}^{n, 2} W^{(0)} \Delta_{t, i}^{n, 2} \widetilde{W}^{(j)}\right], \\
\chi_{i, t, \kappa, n}^{(b)}=2\left(\frac{1}{N} \sum_{j=1}^{N}\left(\beta_{t, \kappa, n}^{(j)}-1\right)^{2}\right) \frac{n}{\sqrt{\left|\widetilde{\mathcal{I}}_{\kappa}^{n}\right|}} \Delta_{t, i-1}^{n} W^{(0)} \Delta_{t, i}^{n} W^{(0)}, \\
\chi_{i, t, \kappa, n}^{(c)}=\frac{1}{N} \frac{n}{\sqrt{\left|\widetilde{\mathcal{I}}_{\kappa}^{n}\right|}} \sum_{j=1}^{N}\left[\left(\beta_{t, \kappa, n}^{(j)}-1\right) \frac{\gamma_{t, \kappa, n}^{(j)}}{\sigma_{t, \kappa, n}^{(0)}} \Delta_{t, i}^{n, 2} W^{(0)} \Delta_{t, i}^{n, 2} B\right],
\end{gathered}
$$

and we note that we have

$$
\frac{1}{N} \sum_{j=1}^{N} \bar{Z}_{t, \kappa, n}^{(j)}=\sqrt{\left|\tilde{\mathcal{I}}_{\kappa}^{n}\right|} \sqrt{N} \widehat{Z}_{t, \kappa, n}^{(a)}+\sqrt{\left|\widetilde{\mathcal{I}}_{\kappa}^{n}\right|}\left(\widehat{Z}_{t, \kappa, n}^{(b)}+\widehat{Z}_{t, \kappa, n}^{(c)}\right) .
$$

Lemma 10. Assume Assumptions $S A$ and $B$ hold. For $n \rightarrow \infty, k_{n} \rightarrow \infty$ and $N \rightarrow \bar{N}$, with $\bar{N} \in(0, \infty]$, we have

$$
\left\{\widehat{Z}_{t, \kappa, n}^{(a)}, \widehat{Z}_{t, \kappa, n}^{(b)}, \widehat{Z}_{t, \kappa, n}^{(c)}\right\}_{t \in \mathcal{T}, \kappa \in \mathcal{K}} \stackrel{\mathcal{L}-s}{\longrightarrow}\left\{\sqrt{\psi_{t, \kappa}^{(a)}} Z_{t, \kappa}^{(a)}, \sqrt{\psi_{t, \kappa}^{(b)}} Z_{t, \kappa}^{(b)}, \sqrt{\psi_{t, \kappa}^{(c)}} Z_{t, \kappa}^{(c)}\right\}_{t \in \mathcal{T}, \kappa \in \mathcal{K}}
$$

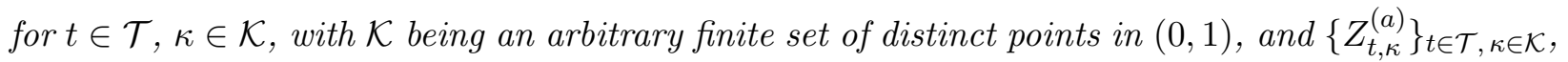
$\left\{Z_{t, \kappa}^{(b)}\right\}_{t \in \mathcal{T}, \kappa \in \mathcal{K}}$ and $\left\{Z_{t, \kappa}^{(c)}\right\}_{t \in \mathcal{T}, \kappa \in \mathcal{K}}$ being three sequences of i.i.d. standard normal random variables defined on an extension of the original probability space and independent of $\mathcal{F}$ and each other. 
Proof of Lemma 10. We denote

$$
\widetilde{\chi}_{i, t, \kappa, n}^{(k)}=\chi_{i, t, \kappa, n}^{(k)}-\mathbb{E}_{t, i}^{n}\left(\chi_{i, t, \kappa, n}^{(k)}\right)+\mathbb{E}_{t, i+1}^{n}\left(\chi_{i+1, t, \kappa, n}^{(k)}\right)-\mathbb{E}_{t, i}^{n}\left(\chi_{i+1, t, \kappa, n}^{(k)}\right), k=a, b, c .
$$

Then, for $k=a, b, c$, we have

$$
\mathbb{E}_{t,\lfloor\kappa n\rfloor-k_{n}}^{n}\left(\sum_{i \in \widetilde{\mathcal{I}}_{\kappa}^{n}}\left(\widetilde{\chi}_{i, t, \kappa, n}^{(k)}-\chi_{i, t, \kappa, n}^{(k)}\right)\right)=0, \mathbb{E}_{t,\lfloor\kappa n\rfloor-k_{n}}^{n}\left(\sum_{i \in \widetilde{\mathcal{I}}_{\kappa}^{n}}\left(\widetilde{\chi}_{i, t, \kappa, n}^{(k)}-\chi_{i, t, \kappa, n}^{(k)}\right)\right)^{2} \leq \frac{K}{k_{n}},
$$

and from here

$$
\sum_{i \in \widetilde{\mathcal{I}}_{\kappa}^{n}}\left(\widetilde{\chi}_{i, t, \kappa, n}^{(k)}-\chi_{i, t, \kappa, n}^{(k)}\right)=o_{p}(1), k=a, b, c .
$$

Therefore, it suffices to prove the convergence result with $\chi_{i, t, \kappa, n}^{(k)}$ replaced by $\widetilde{\chi}_{i, t, \kappa, n}^{(k)}$ in $\widehat{Z}_{t, \kappa, n}^{(k)}$. Such a convergence result will follow by an application of Theorem IX.7.3 of Jacod and Shiryaev (2003) (by noting that $\mathbb{E}_{t, i}^{n}\left(\widetilde{\chi}_{i, t, \kappa, n}^{(k)}\right)=0$ ) if we show the following convergence results:

$$
\begin{gathered}
\sum_{i \in \widetilde{\mathcal{I}}_{\kappa}^{n}} \mathbb{E}_{t, i}^{n}\left(\widetilde{\chi}_{i, t, \kappa, n}^{(a)}\right)^{2} \stackrel{\mathbb{P}}{\longrightarrow} \psi_{t, \kappa}^{(a)}, \sum_{i \in \widetilde{\mathcal{I}}_{\kappa}^{n}} \mathbb{E}_{t, i}^{n}\left(\widetilde{\chi}_{i, t, \kappa, n}^{(b)}\right)^{2} \stackrel{\mathbb{P}}{\longrightarrow} \psi_{t, \kappa}^{(b)}, \sum_{i \in \widetilde{\mathcal{I}}_{\kappa}^{n}} \mathbb{E}_{t, i}^{n}\left(\widetilde{\chi}_{i, t, \kappa, n}^{(c)}\right)^{2} \stackrel{\mathbb{P}}{\longrightarrow} \psi_{t, \kappa}^{(c)}, \\
\sum_{i \in \widetilde{\mathcal{I}}_{\kappa}^{n}} \mathbb{E}_{t, i}^{n}\left(\widetilde{\chi}_{i, t, \kappa, n}^{(k)} \widetilde{\chi}_{i, t, \kappa, n}^{(l)}\right) \stackrel{\mathbb{P}}{\longrightarrow} 0, k, l=a, b, c \text { with } k \neq l, \\
\sum_{i \in \widetilde{\mathcal{I}}_{\kappa}^{n}} \mathbb{E}_{t, i}^{n}\left(\widetilde{\chi}_{i, t, \kappa, n}^{(k)} \Delta_{t, i}^{n} M\right) \stackrel{\mathbb{P}}{\longrightarrow} 0, k=a, b, c,
\end{gathered}
$$

for $M$ being $W^{(0)}$, a component of $B$, or a bounded martingale orthogonal to them (in a martingale sense). The first two convergence results in (151) and (152) follow directly by taking into account that the volatility processes and the beta process all have càdlàg paths. The last convergence result in (153) when $M$ is $W^{(0)}$ or a component of $B$ holds trivially because due to the symmetry of the standard normal distribution, $\mathbb{E}_{t, i}^{n}\left(\widetilde{\chi}_{i, t, \kappa, n}^{(k)} \Delta_{t, i}^{n} M\right)=0$ in this case.

Suppose now that $M$ in (153) is equal to a bounded martingale orthogonal to $W^{(0)}$ and $B$. First, $\mathbb{E}_{t, i}^{n}\left(\widetilde{\chi}_{i, t, \kappa, n}^{(k)} \Delta_{t, i}^{n} M\right)=0$ for $k=a, b$ because $M$ is orthogonal to $W^{(0)}$ and $B$. Second, if $M$ is a discontinuous martingale, we again trivially have $\mathbb{E}_{t, i}^{n}\left(\widetilde{\chi}_{i, t, \kappa, n}^{(k)} \Delta_{t, i}^{n} M\right)=0$ for $k=a, b, c$. Thus, we are left with showing (153) with $k=c$ and $M$ being a continuous bounded martingale that is orthogonal to $W^{(0)}$ and $B$. In this case, we can write

$$
\mathbb{E}_{t, i}^{n}\left(\Delta_{t, i-1}^{n} W^{(0)} \Delta_{t, i}^{n} \widetilde{W}^{(j)} \Delta_{t, i}^{n} M\right)=0
$$

and

$$
\mathbb{E}_{t, i}^{n}\left(\Delta_{t, i}^{n} W^{(0)} \Delta_{t, i}^{n} \widetilde{W}^{(j)} \Delta_{t, i}^{n} M\right)=\mathbb{E}_{t, i}^{n}\left(\Delta_{t, i}^{n} W^{(0)} \Delta_{t, i}^{n} \widetilde{W}^{(j)} Z_{t-1+i / n}^{N}\right)
$$


where $Z_{s}^{N}=\mathbb{E}\left(\Delta_{t, i}^{n} M \mid \widetilde{\mathcal{F}}_{s}^{(N)}\right)$ for $s \in[t-1+(i-1) / n, t-1+i / n]$ and for $\left\{\widetilde{\mathcal{F}}_{t}^{(N)}\right\}_{t \geq 0}$ being the filtration generated by the Brownian motions $W^{(0)}, B$ and $\left\{\widetilde{W}^{(j)}\right\}_{j=1, \ldots, N}$. Note that $Z_{s}^{N}$ is a $\widetilde{\mathcal{F}}^{(N)}$-martingale for $s \in[t-1+(i-1) / n, t-1+i / n]$. Therefore, by a martingale representation theorem, the orthogonality of $M$ to $W^{(0)}$ and $B$, the fact that $W^{(0)}$ and $B$ are independent from each other, and using integration by parts, we have

$$
\begin{aligned}
\mathbb{E}_{t, i}^{n}\left(\Delta_{t, i}^{n} W^{(0)} \Delta_{t, i}^{n} \widetilde{W}^{(j)} Z_{t-1+i / n}^{N}\right) & =\mathbb{E}_{t, i}^{n}\left(\Delta_{t, i}^{n} W^{(0)} \Delta_{t, i}^{n} \widetilde{W}^{(j)} \sum_{j=1}^{N} \int_{t-1+(i-1) / n}^{t-1+i / n} \eta_{s}^{(j)} d \widetilde{W}_{s}^{(j)}\right) \\
& =\mathbb{E}_{t, i}^{n}\left(\sum_{j=1}^{N} \int_{t-1+(i-1) / n}^{t-1+i / n}\left(W_{s}^{(0)}-W_{t-1+(i-1) / n}^{(0)}\right) \eta_{s}^{(j)} d s\right),
\end{aligned}
$$

where $\left\{\eta_{s}^{(j)}\right\}_{j=1, \ldots, N}$ are some $\mathcal{F}_{s}$-adapted processes. From here, using the shorthand notation $\zeta_{t, \kappa, n}^{(j)}=\left(\beta_{t, \kappa, n}^{(j)}-1\right) \widetilde{\sigma}_{t, \kappa, n}^{(j)}$, we can write

$$
\begin{aligned}
& \sum_{j=1}^{N}\left(\zeta_{t, \kappa, n}^{(j)} \sum_{i \in \widetilde{\mathcal{I}}_{\kappa}^{n}} \mathbb{E}_{t, i}^{n}\left(\Delta_{t, i}^{n} W^{(0)} \Delta_{t, i}^{n} \widetilde{W}^{(j)} \Delta_{t, i}^{n} M\right)\right) \\
& \quad=\sum_{i \in \widetilde{\mathcal{I}}_{\kappa}^{n}} \mathbb{E}_{t, i}^{n}\left(\sum_{j=1}^{N} \zeta_{t, \kappa, n}^{(j)} \int_{t-1+(i-1) / n}^{t-1+i / n}\left(W_{s}^{(0)}-W_{t-1+(i-1) / n}^{(0)}\right) \eta_{s}^{(j)} d s\right)
\end{aligned}
$$

From here, by applying Cauchy-Schwarz inequality, we have

$$
\left|\mathbb{E}_{t, i}^{n}\left(\int_{t-1+(i-1) / n}^{t-1+i / n}\left(W_{s}^{(0)}-W_{t-1+(i-1) / n}^{(0)}\right) \eta_{s}^{(j)} d s\right)\right| \leq \frac{K}{\sqrt{n}} \int_{t-1+(i-1) / n}^{t-1+i / n} \sqrt{\mathbb{E}_{t, i}^{n}\left(\eta_{s}^{(j)}\right)^{2}} d s .
$$

Using inequality in means, we have

$$
\sum_{j=1}^{N} \sqrt{\mathbb{E}_{t, i}^{n}\left(\eta_{s}^{(j)}\right)^{2}} \leq K \sqrt{N} \sqrt{\mathbb{E}_{t, i}^{n}\left(\sum_{j=1}^{N}\left(\eta_{s}^{(j)}\right)^{2}\right)}
$$

and therefore by another application of equality in means, we have

$$
\begin{aligned}
& \sum_{j=1}^{N} \int_{t-1+(i-1) / n}^{t-1+i / n} \sqrt{\mathbb{E}_{t, i}^{n}\left(\eta_{s}^{(j)}\right)^{2}} d s \leq K \sqrt{\frac{N}{n}} \sqrt{\mathbb{E}_{t, i}^{n}\left(\int_{t-1+(i-1) / n}^{t-1+i / n} \sum_{j=1}^{N}\left(\eta_{s}^{(j)}\right)^{2} d s\right)} \\
& \leq K \sqrt{\frac{N}{n}} \sqrt{\mathbb{E}_{t, i}^{n}\left(\int_{t-1+(i-1) / n}^{t-1+i / n} d\left\langle Z^{N}, Z^{N}\right\rangle_{s}\right)} \text {. }
\end{aligned}
$$

Applying again inequality in means, we can finally write

$$
\mathbb{E}\left(\sum_{i \in \widetilde{\mathcal{I}}_{\kappa}^{n}} \sum_{j=1}^{N} \int_{t-1+(i-1) / n}^{t-1+i / n} \sqrt{\mathbb{E}_{t, i}^{n}\left(\eta_{s}^{(j)}\right)^{2}} d s\right) \leq K \sqrt{k_{n}} \sqrt{\frac{N}{n}} \sqrt{\sum_{i \in \widetilde{\mathcal{I}}_{\kappa}^{n}} \mathbb{E}\left(\Delta_{t, i}^{n}\left\langle Z^{N}, Z^{N}\right\rangle\right)} .
$$


Now, using the definition of the martingale $Z^{N}$, successive conditioning and Jensen's inequality, we have

$$
\begin{aligned}
\mathbb{E}\left(\Delta_{t, i}^{n}\left\langle Z^{N}, Z^{N}\right\rangle\right) & =\mathbb{E}\left(\mathbb{E}\left(\Delta_{t, i}^{n} M \mid \widetilde{\mathcal{F}}_{t-1+i / n}^{(N)}\right)\right)^{2}-\mathbb{E}\left(\mathbb{E}\left(\Delta_{t, i}^{n} M \mid \widetilde{\mathcal{F}}_{t-1+(i-1) / n}^{(N)}\right)\right)^{2} \\
& \leq 2 \mathbb{E}\left(\Delta_{t, i}^{n} M\right)^{2}
\end{aligned}
$$

Because of the boundedness of the martingale $M$ and its continuity, we therefore have

$$
\sum_{i \in \widetilde{\mathcal{I}}_{\kappa}^{n}} \mathbb{E}\left(\Delta_{t, i}^{n}\left\langle Z^{N}, Z^{N}\right\rangle\right) \leq 2 \mathbb{E}\left(\langle M, M\rangle_{t-1+\frac{\lfloor\kappa n\rfloor}{n}}-\langle M, M\rangle_{t-1+\frac{\lfloor\kappa n\rfloor-k_{n}+1}{n}}\right) \downarrow 0,
$$

as $k_{n} / n \rightarrow 0$.

Combining Lemmas 3,5 -8, we have for $\varpi \in(1 / 4,1 / 2), \varrho \in(0,1 / 2)$ with $\varrho>2-4 \varpi$ :

$$
\widehat{D}_{t, \kappa}-\widehat{B}_{t, \kappa}-D_{t, \kappa}^{N}=\frac{1}{N} \sum_{j=1}^{N} \bar{Z}_{t, \kappa, n}^{(j)}+O_{p}\left(\frac{1}{\alpha_{n}^{4}}\left(\frac{1}{k_{n}} \bigvee \sqrt{\frac{k_{n}}{n}} \bigvee \Delta_{n}^{\varpi}\right)\right)
$$

Moreover, from Lemma 9 and Assumption B, under the same conditions for $\varpi$ and $\varrho$ as above,

$$
\frac{\widehat{\operatorname{Avar}}\left(\widehat{D}_{t, \kappa}^{N}\right)}{\operatorname{Avar}\left(\widehat{D}_{t, \kappa}^{N}\right)} \stackrel{\mathbb{P}}{\longrightarrow} 1, \frac{\left|\widetilde{\mathcal{I}}_{\kappa}^{n}\right| \operatorname{Avar}\left(\widehat{D}_{t, \kappa}^{N}\right)}{\psi_{t, \kappa}^{(a)} / N+\psi_{t, \kappa}^{(b)}+\psi_{t, \kappa}^{(c)}} \stackrel{\mathbb{P}}{\longrightarrow} 1 .
$$

Combining these results with Lemma 10, we get the result of the theorem.

\subsection{Proof of Theorem 2}

We start with showing the counterpart of Lemma 10 in the current context. The result of Lemma 11 below is slightly more restrictive than what we showed in Lemma 10 when $\bar{N}=\infty$. Nevertheless, it suffices for the purposes of proving Theorem 2 .

Lemma 11. Assume Assumptions $S A$ and $B$ hold and let $\left\{\omega_{l}\right\}_{l \in I}$ be Lipschitz real-valued continuous functions on $[0,1]$, where $I$ is a countable set. For $n \rightarrow \infty, k_{n} \rightarrow \infty$ and $N \rightarrow \bar{N}$, with $\bar{N} \in(0, \infty)$, we have:

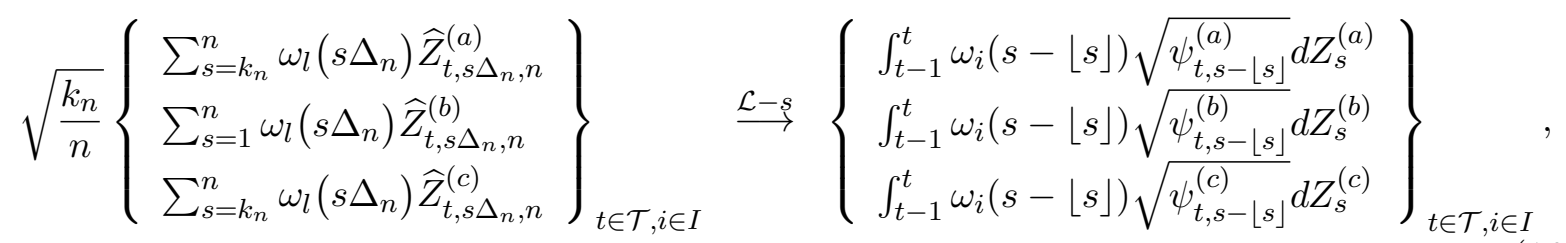

where $Z_{s}^{(a)}, Z_{s}^{(b)}$ and $Z_{s}^{(c)}$ are three independent Brownian motions sequences defined on an extension of the original probability space and independent of $\mathcal{F}$. If in the above setting $\bar{N}=\infty$, then the convergence result in (166) for the sums involving $\widehat{Z}_{t, s \Delta_{n}, n}^{(b)}$ and $\widehat{Z}_{t, s \Delta_{n}, n}^{(c)}$ continues to hold. 
Proof of Lemma 11. Using the notation and the bounds derived in Lemma 10, we have

$$
\sqrt{\frac{k_{n}}{n}} \sum_{s=k_{n}}^{n} \sum_{i \in \widetilde{\mathcal{I}}_{s \Delta_{n}}} \omega_{l}\left(s \Delta_{n}\right)\left(\widetilde{\chi}_{t, i, s \Delta_{n}, n}^{(k)}-\chi_{t, i, s \Delta_{n}, n}^{(k)}\right)=O_{p}\left(1 / \sqrt{k_{n}}\right), k=a, b, c .
$$

From here the proof of the lemma follows exactly the same steps as corresponding ones in the proof of Lemma 10.

Combining Lemmas 3, 5-8, we have for $\varpi \in(1 / 4,1 / 2), \varrho \in(0,1 / 2)$ with $\varrho>2-4 \varpi$ and for $\omega$ as in Lemma 11 above:

$$
\begin{aligned}
& \frac{1}{n-k_{n}+1} \sum_{s=k_{n}}^{n}\left[\omega\left(s \Delta_{n}\right)\left(\widehat{D}_{t, s \Delta_{n}}^{N}-\widehat{B}_{t, s \Delta_{n}}^{N}\right)\right]-\int_{t-1}^{t} \omega(s-t+1) D_{t, s-\lfloor s\rfloor}^{N} d s \\
& =\frac{1}{n-k_{n}+1} \sum_{s=k_{n}}^{n} \omega\left(s \Delta_{n}\right) \frac{1}{N} \sum_{j=1}^{N} \bar{Z}_{t, s \Delta_{n}, n}^{(j)}+O_{p}\left(\frac{1}{\alpha_{n}^{4}}\left(\frac{1}{k_{n}^{3 / 2}} \bigvee\left(\frac{k_{n}}{n}\right)^{1-\iota} \bigvee \Delta_{n}^{2 \varpi}\right)\right) .
\end{aligned}
$$

Moreover, from Lemma 9 and Assumption B, under the same conditions for $\varpi$ and $\varrho$ as above, we have for arbitrary $\omega, \omega^{\prime}$ that are Lipschitz real-valued continuous functions on $[0,1]$ :

$$
\frac{\sum_{s=1}^{n}\left[\bar{\omega}^{n}(s) \bar{\omega}^{\prime} n(s)\left(\widehat{\operatorname{Avar}}\left(\widehat{D}_{t,\left(s \vee k_{n}\right) \Delta_{n}}^{N}\right)+\widehat{\operatorname{Avar}}\left(\widehat{D}_{t,\left(s+k_{n} \wedge n\right) \Delta_{n}}^{N}\right)\right)\right]}{\sum_{s=1}^{n}\left[\bar{\omega}^{n}(s) \bar{\omega}^{\prime} n(s)\left(\operatorname{Avar}\left(\widehat{D}_{t,\left(s \vee k_{n}\right) \Delta_{n}}^{N}\right)+\operatorname{Avar}\left(\widehat{D}_{t,\left(s+k_{n} \wedge n\right) \Delta_{n}}^{N}\right)\right)\right]} \stackrel{\mathbb{P}}{\longrightarrow} 1,
$$

and

$$
\frac{1}{2} \frac{k_{n}}{n} \frac{\sum_{s=1}^{n}\left[\bar{\omega}^{n}(s) \bar{\omega}^{\prime} n(s)\left(\operatorname{Avar}\left(\widehat{D}_{t,\left(s \vee k_{n}\right) \Delta_{n}}^{N}\right)+\operatorname{Avar}\left(\widehat{D}_{t,\left(s+k_{n} \wedge n\right) \Delta_{n}}^{N}\right)\right)\right]}{\int_{t-1}^{t}\left[\omega(s-t+1) \omega^{\prime}(s-t+1)\left(\psi_{t, s-\lfloor s\rfloor}^{(a)} / N+\psi_{t, s-\lfloor s\rfloor}^{(b)}+\psi_{t, s-\lfloor s\rfloor}^{(c)}\right)\right] d s} \stackrel{\mathbb{P}}{\longrightarrow} 1,
$$

where we define $\bar{\omega}^{n}(i)$ and $\bar{\omega}^{\prime}(i)$, for $i=1, \ldots, n$, from $\omega$ and $\omega^{\prime}$ exactly as in (30).

Furthermore, the above two convergences hold uniformly for $\omega, \omega^{\prime}$ belonging to the set of weighting functions of the theorem. Overall, the above results, together with Lemma 11 imply the convergence result of the theorem holds finite-dimensionally, i.e., for any finite set of points in $\mathcal{U}$. Therefore, we are left with showing tightness of the sequence in the space of continuous functions on $\mathcal{U}$ equipped with the uniform topology. For this, we make use of Theorem 12.3 of Billingsley (2013) and Lemmas 3, 5-8 as well as the smoothness of $\omega_{u}(z)$ in $u$ assumed in the statement of the theorem.

\subsection{Proof of Corollary 1}

Part (a) follows from Theorem 2 while part (b) follows from Theorem 1 of Bierens (1982). 


\subsection{Proof of Thoerem 3}

Throughout this proof, $\|f\|=\langle f, f\rangle$ is the norm of $f \in \mathcal{L}^{2}(w)$. We start the proof with denoting the function

$$
g(u, x, y)=e^{i u \frac{y}{x}+i u}, \text { for } u, x, y \in \mathbb{R} .
$$

Using Taylor expansion, we then have the decomposition

$$
g\left(u, \widehat{V}_{t, \kappa}^{(0, j)}, \widehat{C}_{t, \kappa}^{(j)}(2)\right)-g\left(u, \widetilde{V}_{t, \kappa}^{(0)}, \widetilde{C}_{t, \kappa}^{(j)}\right)=Z_{t, \kappa, n}^{(j)}(u)+R_{t, \kappa}^{(j)}(u)
$$

where

$$
Z_{t, \kappa, n}^{(j)}(u)=\nabla_{x} g\left(u, \widetilde{V}_{t, \kappa}^{(0)}, \widetilde{C}_{t, \kappa}^{(j)}\right)\left(\widehat{V}_{t, \kappa}^{(0, j)}-\widetilde{V}_{t, \kappa}^{(0)}\right)+\nabla_{y} g\left(u, \widetilde{V}_{t, \kappa}^{(0)}, \widetilde{C}_{t, \kappa}^{(j)}\right)\left(\widehat{C}_{t, \kappa}^{(j)}(2)-\widetilde{C}_{t, \kappa}^{(j)}\right),
$$

and the residual term $R_{t, \kappa}^{(j)}(u)$ satisfies

$$
\left|R_{t, \kappa}^{(j)}(u)\right| \leq K(|u| \vee 1) 1_{\left\{\left|\widehat{V}_{t, \kappa}^{(0, j)}\right| \leq \alpha_{n}\right\}}+K\left(|u|^{2} \vee 1\right)\left(\left(\widehat{V}_{t, \kappa}^{(0, j)}-\widetilde{V}_{t, \kappa}^{(0)}\right)^{2}+\left(\widehat{C}_{t, \kappa}^{(j)}(2)-\widetilde{C}_{t, \kappa}^{(j)}\right)^{2}\right),
$$

with a constant $K$ that does not depend on $n, j$ and $u$. We further denote

$$
\begin{aligned}
& \bar{Z}_{t, \kappa, n}^{(j)}(u)=e^{i u \beta_{t, \kappa, n}^{(j)}} i u\left(\beta_{t, \kappa, n}^{(j)}-1\right)^{2} \frac{n}{\left|\widetilde{\mathcal{I}}_{\kappa}^{n}\right|} \sum_{i \in \widetilde{\mathcal{I}}_{\kappa}^{n}} \Delta_{t, i-1}^{n} W^{(0)} \Delta_{t, i}^{n} W^{(0)} \\
& +\frac{1}{2} e^{i u \beta_{t, \kappa, n}^{(j)}} i u\left(\beta_{t, \kappa, n}^{(j)}-1\right)\left(\frac{n}{\left|\widetilde{\mathcal{I}}_{\kappa}^{n}\right|} \sum_{i \in \widetilde{\mathcal{I}}_{\kappa}^{n}} \frac{\gamma_{t, \kappa, n}^{(j)}}{\sigma_{t, \kappa, n}^{(0)}} \Delta_{t, i}^{n, 2} W^{(0)} \Delta_{t, i}^{n, 2} B+\frac{n}{\left|\widetilde{\mathcal{I}}_{\kappa}^{n}\right|} \sum_{i \in \widetilde{\mathcal{I}}_{\kappa}^{n}} \frac{\widetilde{\sigma}_{t, \kappa, n}^{(0)}}{\sigma_{t, \kappa, n}^{(0)}} \Delta_{t, i}^{n, 2} W^{(0)} \Delta_{t, i}^{n, 2} \widetilde{W}^{(j)}\right) .
\end{aligned}
$$

Using Lemmas 1-3 and Lemma 8 and the exponential tail decay of the weighting function $w$, we have

$$
\mathbb{E}\left\|\frac{1}{N} \sum_{j=1}^{N} R_{t, \kappa}^{(j)}\right\| \leq \frac{K}{\alpha_{n}^{2}}\left(\frac{1}{k_{n}} \bigvee \frac{k_{n}}{n}\right),
$$

where we have made use of the fact that $\varpi>1 / 4$. Similarly, using Lemmas $1-3$, we have that

$$
\mathbb{E}\left\|\frac{1}{N} \sum_{j=1}^{N}\left(Z_{t, \kappa, n}^{(j)}-\bar{Z}_{t, \kappa, n}^{(j)}\right)\right\| \leq \frac{K}{\alpha_{n}^{2}}\left(\frac{1}{k_{n}} \bigvee \frac{k_{n}}{n}\right) \leq K\left(\Delta_{n}^{2 \varpi} \bigvee \sqrt{\frac{k_{n}}{n}} \bigvee \frac{1}{k_{n}}\right) .
$$

Given the rate condition on the sequence $k_{n}$, we are left with showing

$$
\sqrt{k_{n}} \frac{1}{N} \sum_{j=1}^{N} \bar{Z}_{t, \kappa, n}^{(j)} \stackrel{\mathcal{L}-s}{\longrightarrow} \mathcal{Z}_{t, \kappa}
$$


with $\mathcal{Z}_{t, \kappa}$ being the limit in the statement of the theorem. Using Bessel's inequality and dominated convergence we have

$$
\mathbb{E}\left(\sum_{i>I}\left\langle\frac{k_{n}}{N} \sum_{j=1}^{N} \bar{Z}_{t, \kappa, n}^{(j)}, e_{i}\right\rangle^{2}\right) \rightarrow 0, \quad \text { as } I \rightarrow \infty,
$$

where $\left\{e_{i}\right\}_{i \geq 1}$ denotes an orthonormal basis in $\mathcal{L}^{2}(w)$. This means that the sequence is asymptotically finite-dimensional, see 1.8 in Vaart and Wellner (1996). Therefore, the limit result of the theorem will follow from Theorem 1.8.4 in Vaart and Wellner (1996) if we can establish

$$
\left\langle\frac{\sqrt{k_{n}}}{N} \sum_{j=1}^{N} \bar{Z}_{t, \kappa, n}^{(j)}, h\right\rangle \stackrel{\mathcal{L}-s}{\longrightarrow}\left\langle\mathcal{Z}_{t, \kappa}, h\right\rangle,
$$

for $\mathcal{Z}_{t, \kappa}$ denoting the limit of Theorem 1 and $h$ an arbitrary element in $\mathcal{L}^{2}(w)$. This convergence follows by an application of Lemma 11.

\section{References}

Barndorff-Nielsen, O. E., And N. Shephard (2004a): "Econometric analysis of realized covariation: High frequency based covariance, regression, and correlation in financial economics," Econometrica, 72(3), 885-925.

Barndorff-Nielsen, O. E., and N. Shephard (2004b): "Power and bipower variation with stochastic volatility and jumps," Journal of Financial Econometrics, 2, 1-37.

Bierens, H. J. (1982): "Consistent model specification tests," Journal of Econometrics, 20(1), $105-134$.

Bierens, H. J., And W. Ploberger (1997): "Asymptotic theory of integrated conditional moment tests," Econometrica, 65(5), 1129-1151.

Billingsley, P. (2013): Convergence of probability measures. John Wiley \& Sons.

Black, F. (1972): "Capital market equilibrium with restricted borrowing," Journal of Business, $45(3), 444-455$.

Black, F., M. C. Jensen, and M. Scholes (1972): "The capital asset pricing model: Some empirical tests," Studies in the theory of capital markets, 81(3), 79-121.

Bollerslev, T., S. Z. Li, and V. Todorov (2016): "Roughing up beta: Continuous versus discontinuous betas and the cross section of expected stock returns," Journal of Financial Economics, 120(3), 464-490. 
Bollerslev, T., And V. Todorov (2011): "Estimation of jump tails," Econometrica, 79(6), $1727-1783$.

Carhart, M. M. (1997): "On persistence in mutual fund performance," Journal of Finance, $52(1), 57-82$.

Connor, G., M. Hagmann, and O. Linton (2012): "Efficient semiparametric estimation of the Fama-French model and extensions," Econometrica, 80(2), 713-754.

Fama, E. F., And K. R. French (1996): "Multifactor explanations of asset pricing anomalies," Journal of Finance, 51(1), 55-84.

Ferson, W. E., And C. R. Harvey (1999): "Conditioning variables and the cross section of stock returns," Journal of Finance, 54(4), 1325-1360.

Frazzini, A., And L. H. Pedersen (2014): "Betting against beta," Journal of Financial Economics, 111(1), 1-25.

Gagliardini, P., E. Ossola, and O. Scaillet (2016): "Time-varying risk premium in large cross-sectional equity data sets," Econometrica, 84(3), 985-1046.

Gospodinov, N., R. Kan, and C. Robotti (2009): "Misspecification-robust inference in linear asset pricing models with irrelevant risk factors," Review of Financial Studies, 27, 2139-2170.

Hansen, L. P., and S. F. Richard (1987): "The role of conditioning information in deducing testable restrictions implied by dynamic asset pricing models," Econometrica, 55(3), 587-613.

Jacod, J., And P. Protter (2012): Discretization of processes. Springer-Verlag, Berlin.

Jacod, J., And A. ShiRyAev (2003): Limit theorems for stochastic processes. Springer-Verlag, Berlin, 2nd edn.

Jaganathan, R., And Z. WAng (1998): "An asymptotic theory for estimating beta-pricing Models using cross-sectional regression," Journal of Finance, 53, 1285-1309.

Jagannathan, R., AND Z. WANG (1996): "The conditional CAPM and the cross-section of expected returns," Journal of Finance, 51(1), 3-53.

Jegadeesh, N., and S. Titman (1993): "Returns to buying winners and selling losers: Implications for stock market efficiency," Journal of Finance, 48(1), 65-91. 
Kan, R., And C. Zhang (1999): "Two-pass tests of asset pricing models with useless factors," Journal of Finance, 54, 204-235.

Kleibergen, F. (2009): "Tests of risk premia in linear factor models," Journal of Econometrics, $149,149-173$.

Li, J., V. Todorov, and G. TAuchen (2017): "Adaptive estimation of continuous-time regression models using high-frequency data," Journal of Econometrics, 200(1), 36-47.

Lou, D., C. Polk, and S. Skouras (2019): "A tug of war: overnight versus intraday expected returns," Journal of Financial Economics.

Mancini, C. (2001): "Disentangling the jumps of the diffusion in a geometric jumping Brownian motion," Giornale dell'Istituto Italiano degli Attuari, 64(19-47), 44.

(2009): "Non-parametric threshold estimation for models with stochastic diffusion coefficient and jumps," Scandinavian Journal of Statistics, 36(2), 270-296.

Mykland, P., And L. Zhang (2009): "Inference for continuous semimartingales observed at high frequency," Econometrica, 77, 1403-1445.

Mykland, P. A., And L. Zhang (2017): "Assessment of uncertainty in high frequency data: The observed asymptotic variance," Econometrica, 85(1), 197-231.

Novy-Marx, R., And M. Velikov (2018): "Betting against betting against beta," SSRN working paper.

Shanken, J. (1990): "Intertemporal asset pricing: An empirical investigation," Journal of Econometrics, 45(1-2), 99-120.

Shanken, J. (1992): "On the estimation of beta pricing models," Review of Financial Studies, 5, 1-33.

VAART, A. W., AND J. Wellner (1996): Weak convergence and empirical processes. Springer, New York. 